Supporting Information

\title{
High-Throughput Approach toward the Development of a Mizoroki-Heck Reaction to Access Tricyclic Spirolactones
}

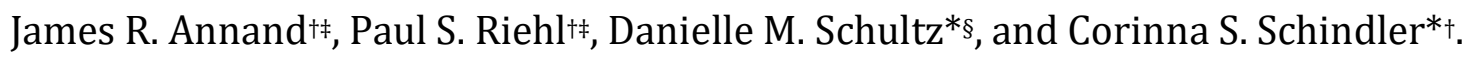

†Department of Chemistry, Willard Henry Dow Laboratory, University of Michigan, 930 North University Ave., Ann Arbor, Michigan 48109, United States.

\$Process Research and Development, Merck \& Co., Inc., Rahway, New Jersey 07065, United States. 


\section{Table of Contents}

I. Ligands for Screening Heck Reactions .................................................... 33

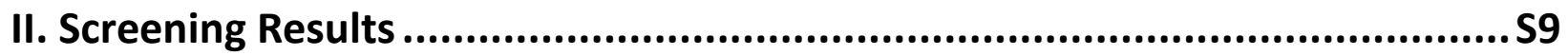

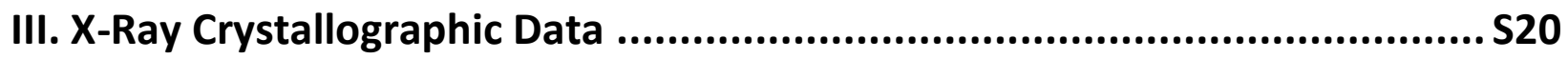

IV. ${ }^{1} \mathrm{H}$ and ${ }^{13} \mathrm{C}$ NMR for Synthetic Compounds ........................................... S33

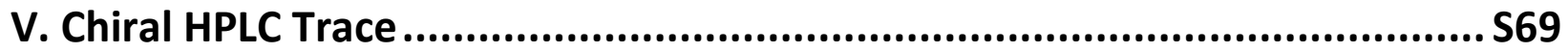




\section{Ligands for Screening Heck Reactions}
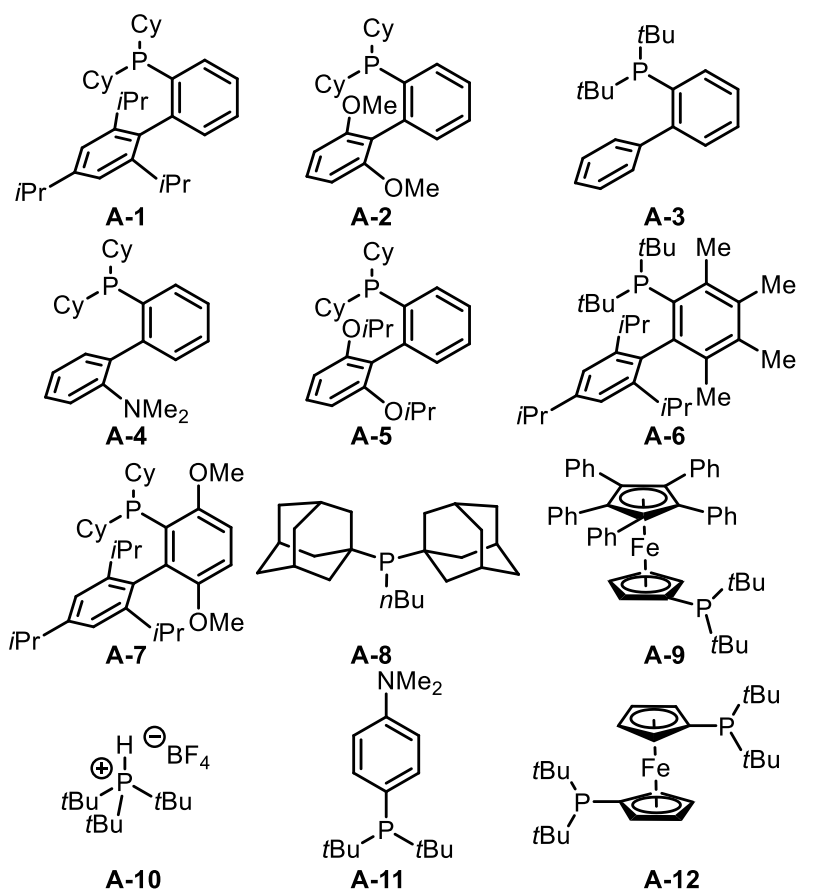

Figure S1. Kit A: Merck collection of phosphine ligands selected for previous success in promoting cross coupling reactions. 

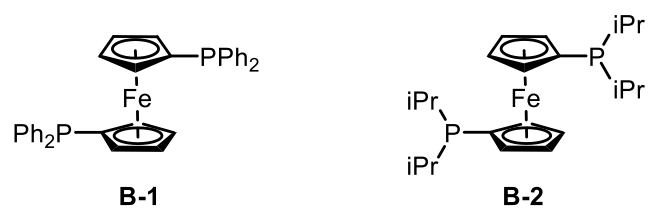

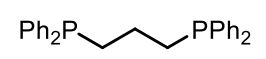<smiles>Pc1ccccc1</smiles>

B-4
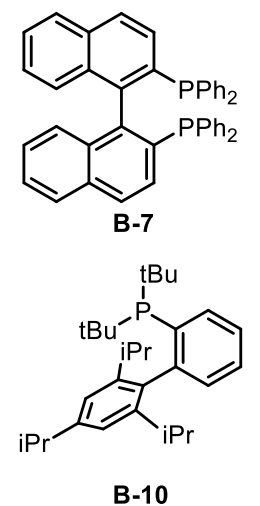

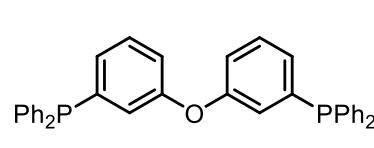

B-5

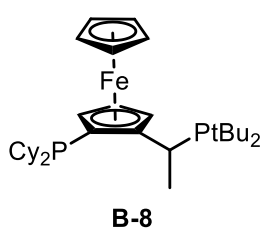

$\Theta \mathrm{BF}_{4}$

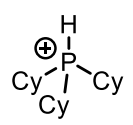

B-11

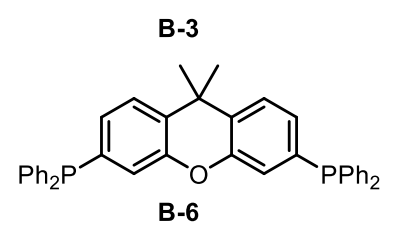

(B)

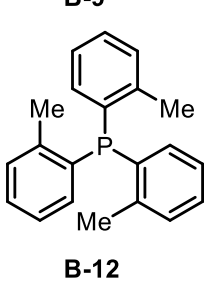

Figure S2. Kit B: Merck collection of phosphine ligands selected for success in promoting cross coupling reactions. 

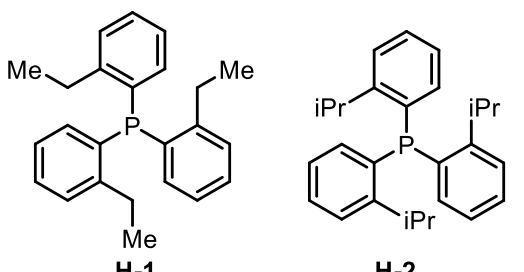<smiles>Cc1ccccc1P(c1ccccc1C(C)C)c1ccccc1C(C)C</smiles><smiles>Cc1cc(F)ccc1P(c1ccc(F)cc1C)c1ccc(F)cc1C</smiles><smiles>Cc1ccc(F)cc1P(c1cc(F)ccc1C)c1cc(F)ccc1C</smiles><smiles>Cc1cccc(C)c1P(c1c(C)cccc1F)c1c(C)cccc1F</smiles><smiles>Cc1cc(Cl)ccc1P(c1ccc(Cl)cc1C)c1ccc(Cl)cc1C</smiles><smiles>Cc1cc(C(F)(F)F)ccc1P(c1ccc(C(F)(F)F)cc1C)c1ccc(C(F)(F)F)cc1C</smiles><smiles>Cc1ccc(C(F)(F)F)cc1P(c1cc(C(F)(F)F)ccc1C)c1cc(C(F)(F)F)ccc1C</smiles><smiles>COc1ccc(C)c(P(c2cc(OC)ccc2C)c2cc(OC)ccc2C)c1</smiles><smiles>CNc1ccc(P(c2ccc(N(C)C)cc2C)c2ccc(N(C)C)cc2C)c(C)c1</smiles><smiles>Cc1ccccc1P(c1ccccn1)c1ccccc1C</smiles><smiles>Cc1ccccc1P(c1ccccc1C)c1ccccc1C(C)(C)C=O</smiles><smiles>Cc1ccccc1P(c1ccccc1)c1ccccc1C</smiles>

H-12<smiles>Cc1cc(C)c(P(c2ccccc2C)c2c(C)cc(C)cc2C)c(C)c1</smiles><smiles>CCc1ccccc1P(c1ccccc1C)c1ccccc1CC</smiles><smiles>Cc1cc(C)c(P(c2ccccc2C)c2ccccc2C)c(C)c1</smiles>

H-17

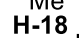

$\mathrm{Me}$

H-19

$\mathrm{CF}_{3}$<smiles>Cc1ccc(P(c2ccc(F)cc2C)C(C)(C)C)c(C)c1</smiles>

H-21

H-22

H-23<smiles>Cc1ccc(F)cc1P(c1cc(F)ccc1C)C(C)(C)C</smiles><smiles>Cc1cc(C)c(P(c2c(C)cc(C)cc2C)C(C)(C)C)c(C)c1</smiles>

H-24

Figure S3. Kit C: Merck collection of monodentate phosphine ligands for the promotion of Mizoroki-Heck reactions. 


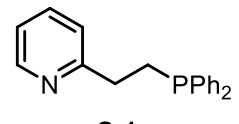

S-1

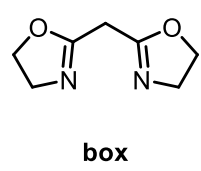

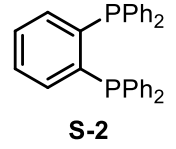

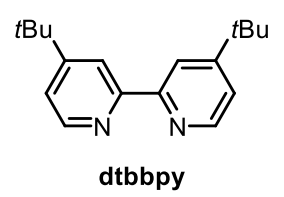<smiles>[18OH]CCP[TeH4]</smiles>

S-3

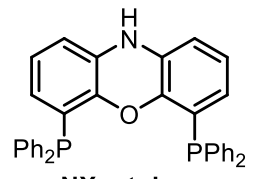

NXantphos

Figure S4. Additional ligands used to evaluate Mizoroki-Heck reactions. 


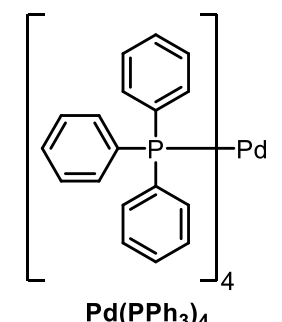<smiles>c1ccc(P(c2ccccc2)c2ccccc2)cc1</smiles><smiles>C1CCC(P(C2CCCCC2)C2CCCCC2)CC1</smiles><smiles>CC(C)(C)P(C(C)(C)C)C(C)(C)C</smiles><smiles>Cc1ccc(C(F)(F)F)cc1P(c1cc(C(F)(F)F)ccc1C)c1cc(C(F)(F)F)ccc1C</smiles><smiles>Cc1ccc(F)cc1P(c1cc(F)ccc1C)c1cc(F)ccc1C</smiles>

$C y_{3} P$

$t \mathrm{Bu}_{3} \mathrm{P}$<smiles>Pc1ccccc1</smiles><smiles>Pc1ccccc1</smiles><smiles>CC1(C)c2ccc(P)cc2Oc2cc(Pc3ccccc3)ccc21</smiles><smiles>c1ccc(Pc2ccc(Pc3ccccc3)cc2)cc1</smiles>

dppf<smiles>Pc1cccc(Pc2ccccc2-c2ccccc2)c1P</smiles>

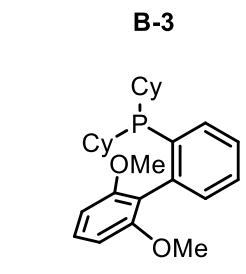

B-4

B-6

BIPHEP<smiles>CC(C)c1cc(C(C)C)c(-c2ccccc2[PH](Cl)(Cl)[I-])c(C(C)C)c1</smiles>

A-1<smiles>CC(C)c1cc(C(C)C)c(C(C)C)c(P(c2ccccc2C(C)C)C(C)(C)C)c1</smiles>

B-10

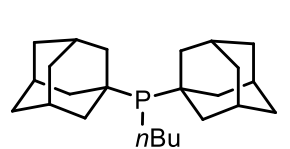<smiles>Cc1ccccc1P(c1ccccc1C)c1ccccc1C</smiles><smiles>c1ccc(CCc2ccccn2)cc1</smiles><smiles>Cc1c(C)c(C(C)(C)C)c(-c2c(C(C)C)cc(C(C)C)cc2C(C)C)c(C(C)(C)C(C)(C)C)c1C</smiles>

A-8

B-12<smiles>CCc1ccccc1P(c1ccccc1CC)c1ccccc1CC</smiles><smiles>CC(C)(C)P(C1=CC=CC1[PH](c1ccccc1)(c1ccccc1)c1ccccc1-c1ccccc1)C(C)(C)C</smiles>

A-9<smiles>Pc1cccc(P)c1P</smiles><smiles></smiles>

S-3

H-1<smiles>Cc1cc(C)c(P(c2ccccc2C)c2ccccc2C)c(C)c1</smiles>

H-16

Figure S5. Kit D: ligands used to evaluate Mizoroki-Heck reactions with Pd. 

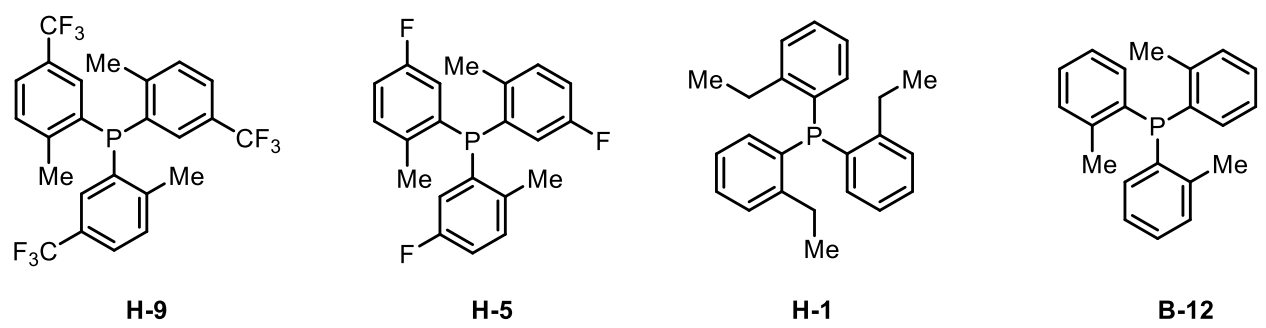

Figure S6. Kit E: 4 ligands initially identified to promote Heck reaction with Pd. 


\section{Screening Results}

Table S1: Mizoroki-Heck Optimization: $\mathrm{Pd}(\mathrm{OAc})_{2}$, Merck A/B Ligands (Manuscript Table 1, Entry 2)

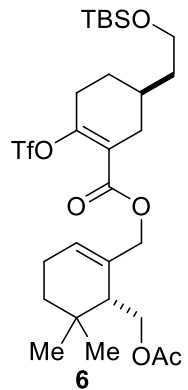

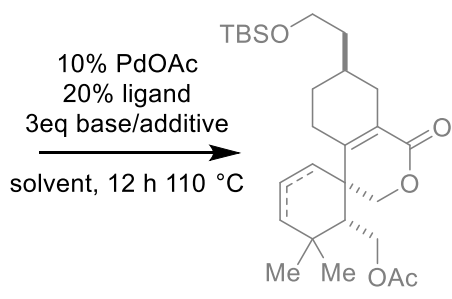

27<smiles>CC(=O)OCCC1CCCC(COCC(=O)OCC2=CCCCC2(C)C)C1</smiles>

28

\begin{tabular}{|c|c|c|c|c|c|c|c|c|c|c|c|c|c|}
\hline \multirow{5}{*}{ DMA } & \multicolumn{12}{|c|}{ Ligand used } & \multirow{5}{*}{$\begin{array}{l}\mathrm{Ag}_{3} \mathrm{PO}_{4} \\
\mathrm{Cy}_{2} \mathrm{NMe} \text { with } \mathrm{AgNO}_{3}\end{array}$} \\
\hline & A-1 & A-2 & $A-3$ & $A-4$ & $A-5$ & A-6 & A-7 & $A-8$ & $A-9$ & A-10 & A-11 & A-12 & \\
\hline & B-1 & B-2 & B-3 & B-4 & B-5 & $B-6$ & B-7 & $B-8$ & B-9 & B-10 & B-11 & B-12 & \\
\hline & A-1 & A-2 & $A-3$ & $A-4$ & $A-5$ & A-6 & $A-7$ & $A-8$ & $A-9$ & A-10 & A-11 & A-12 & \\
\hline & B-1 & B-2 & B-3 & B-4 & B-5 & B-6 & B-7 & B-8 & B-9 & B-10 & B-11 & B-12 & \\
\hline \multirow{4}{*}{ leCN } & A-1 & $A-2$ & $A-3$ & $A-4$ & $A-5$ & A- 6 & $A-7$ & $A-8$ & $A-9$ & A-10 & A-11 & A-12 & \multirow{4}{*}{$\begin{array}{l}\mathrm{Ag}_{3} \mathrm{PO}_{4} \\
\mathrm{Cy}_{2} \mathrm{NMe} \text { with } \mathrm{AgNO}_{3}\end{array}$} \\
\hline & B-1 & B-2 & B-3 & B-4 & B-5 & B-6 & B-7 & $B-8$ & B-9 & B-10 & B-11 & B-12 & \\
\hline & A-1 & $A-2$ & $A-3$ & $A-4$ & $A-5$ & A-6 & $A-7$ & $A-8$ & $A-9$ & A-10 & $A-11$ & A-12 & \\
\hline & B-1 & B-2 & B-3 & B-4 & B-5 & B-6 & B-7 & B-8 & B-9 & B-10 & B-11 & B-12 & \\
\hline
\end{tabular}

$\%$ conversions

\begin{tabular}{|rrrrrrrrrrrr|}
\hline 88.3 & 17.2 & 39.6 & 33.4 & 25.9 & 46.2 & 55.1 & 52.4 & 56.9 & 52 & 14.9 & 20.4 \\
39.5 & 23.3 & 14.5 & -25 & 8.82 & 6.06 & 8.85 & -8.3 & -67 & -43 & 13.8 & -1.6 \\
\hline 58.7 & -38 & 62.6 & -19 & -11 & 9.66 & 45.3 & -5 & 53.9 & 24 & 42 & -1.4 \\
55.9 & 35.9 & 58.8 & 49.9 & 74.4 & 52.7 & 69 & 31.1 & 69.5 & 50 & 61.2 & 56 \\
\hline 50.5 & 56.6 & 46.7 & 35.9 & 12.1 & 45.4 & 62.6 & 52.1 & 58.6 & 45 & 42.9 & 21.7 \\
47 & 42.1 & 47.8 & 50 & 69.4 & 53.6 & 56.6 & 49.3 & 36.9 & 30 & 12.2 & 26 \\
\hline 65.7 & 56.4 & 67.5 & 50.1 & 55.4 & 65.1 & 62.5 & 78.1 & 62.8 & 49 & 63 & 54.2 \\
51.5 & 45.6 & 65.1 & 58.7 & 100 & 48 & 57.4 & 63.4 & 65.9 & 57 & 65.9 & 64.3 \\
\hline
\end{tabular}

$\%$ yield $\mathbf{3 0}$

\begin{tabular}{|rrrrrrrrrrrr|}
\hline 0 & 0 & 0 & 0 & 0 & 0 & 0 & 0 & 0 & 0 & 0 & 0 \\
0 & 0 & 0 & 0 & 0 & 0 & 0 & 0 & 0 & 0 & 0 & 0 \\
0 & 0 & 0 & 0 & 0 & 0 & 5.85 & 0 & 0 & 0 & 0 & 0 \\
0 & 0 & 0 & 0 & 0 & 0 & 0 & 0 & 5.97 & 0 & 0 & 0 \\
0 & 0 & 0 & 0 & 0 & 0 & 0 & 0 & 8.76 & 0 & 0 & 0 \\
0 & 0 & 0 & 0 & 0 & 0 & 0 & 0 & 0 & 0 & 0 & 0 \\
0 & 0 & 5.31 & 0 & 0 & 6.78 & 0 & 4.57 & 7.42 & 0 & 0 & 0 \\
7.06 & 5.11 & 4.58 & 0 & 3.7 & 0 & 5.94 & 0 & 7.4 & 0 & 0 & 6.59 \\
\hline
\end{tabular}

Yield and conversions determined by UPLC/MS ditbubiphenyl as internal standard. No 28 was detected in any case. Reactions set up according to general procedure for Mizoroki-Heck screening A. 
Table S2: Mizoroki-Heck Optimization: $\mathrm{Pd}(\mathrm{OAc})_{2}$ Merck Heck Ligands (Manuscript Table 1, Entry 3)<smiles>CC(=O)OC[C@@H]1C(COC(=O)C2=C(O)CCC(CCOC(C)C)C2)=CCCC1(C)C</smiles>

6

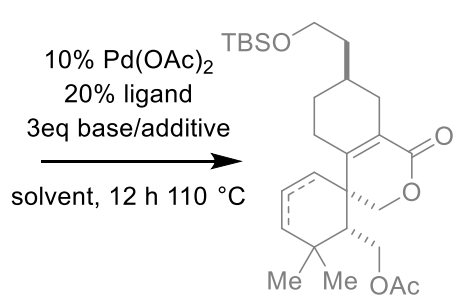

27<smiles>CC(=O)OCC1CCC=CC1COC(=O)C1=CCCC(C)(C)[C@@H]1CO[Sb]</smiles>

28

\begin{tabular}{|c|c|c|c|c|c|c|c|c|c|c|c|c|}
\hline & & & & & & ligan & & & & & & \\
\hline & H-1 & H-2 & $\mathrm{H}-3$ & H-4 & H-5 & H-6 & $\mathrm{H}-7$ & H-8 & H-9 & $\mathrm{H}-10$ & H-11 & $\mathrm{H}-12$ \\
\hline & H-13 & H-14 & H-15 & H-16 & H-17 & H-18 & H-19 & H-20 & H-21 & $\mathrm{H}-22$ & $\mathrm{H}-23$ & H-24 \\
\hline $\mathrm{Cy}_{2} \mathrm{NMe}$ & H-1 & $\mathrm{H}-2$ & $\mathrm{H}-3$ & $\mathrm{H}-4$ & H-5 & H-6 & H-7 & H-8 & H-9 & $\mathrm{H}-10$ & H-11 & $\mathrm{H}-12$ \\
\hline with $\mathrm{AgNO}_{3}$ & H-13 & H-14 & H-15 & H-16 & H-17 & $\mathrm{H}-18$ & H-19 & H-20 & H-21 & $\mathrm{H}-22$ & $\mathrm{H}-23$ & H-24 \\
\hline & H-1 & $\mathrm{H}-2$ & $\mathrm{H}-3$ & $\mathrm{H}-4$ & H-5 & H-6 & H-7 & $\mathrm{H}-8$ & $\mathrm{H}-9$ & H-10 & H-11 & $\mathrm{H}-12$ \\
\hline & H-13 & H-14 & H-15 & H-16 & H-17 & H-18 & H-19 & H-20 & H-21 & $\mathrm{H}-22$ & $\mathrm{H}-23$ & H-24 \\
\hline $\mathrm{Cy}_{2} \mathrm{NMe}$ & H-1 & $\mathrm{H}-2$ & $\mathrm{H}-3$ & $\mathrm{H}-4$ & H-5 & H-6 & $\mathrm{H}-7$ & $\mathrm{H}-8$ & H-9 & $\mathrm{H}-10$ & $\mathrm{H}-11$ & $\mathrm{H}-12$ \\
\hline with $\mathrm{AgNO}_{3}$ & H-13 & H-14 & H-15 & H-16 & $\mathrm{H}-17$ & $\mathrm{H}-18$ & H-19 & $\mathrm{H}-20$ & H-21 & $\mathrm{H}-22$ & $\mathrm{H}-23$ & H-24 \\
\hline
\end{tabular}

$\%$ conversion

\begin{tabular}{|llllllllllll|}
46.3 & 28.6 & 40.2 & 35.6 & 30.4 & 1.45 & 30.8 & 19.3 & 32.8 & 50 & 17.6 & 24.9 \\
\hline
\end{tabular}

\begin{tabular}{lllllll|llllll}
37.7 & 31.4 & 56.7 & 39 & 14 & 35.6 & 7.18 & 40.3 & 31.8 & 41 & 18.2 & 5.72
\end{tabular}

$\begin{array}{llllllllllll}55.2 & 52.8 & 44.3 & 45.5 & 34.5 & 42 & 50.9 & 27.3 & 56.2 & 52 & 31.4 & 21.4\end{array}$

\begin{tabular}{llllllllllll}
50 & 25 & 75.1 & 62.2 & 24 & 45.3 & 42.8 & 40.6 & 44.4 & 26 & 27.3 & 55.3 \\
\hline 46.2 & 33.5 & 21.1 & 26.6 & 0.3 & 26.2 & 37.9 & 18.5 & 7.09 & 1.2 & 29 & 32.5
\end{tabular}

\begin{tabular}{rrrrrrrrrrrr|}
46.2 & 33.5 & 21.1 & 26.6 & 10.3 & 26.2 & 37.9 & 18.5 & 7.09 & 1.2 & 29 & 32.5 \\
\hline & 12.5 & 50.5 & 18.4 & 34.6 & 38.7 & 31.1 & 16.4 & 10.1 & 28 & 10.4 & 29.2
\end{tabular}

\begin{tabular}{lllllllllllll}
34 & 12.5 & 50.5 & 18.4 & 34.6 & 38.7 & 31.1 & 16.4 & 10.1 & 28 & 10.4 & 29.2 \\
\hline
\end{tabular}

$\begin{array}{llllllllllll}59.4 & 41.8 & 58.1 & 58.6 & 44.5 & 54.4 & 58.6 & 51.2 & 45.2 & 41 & 53 & 53.7\end{array}$

\begin{tabular}{|r|r|r|r|r|rrrrrrrr}
65.1 & 47.6 & 81.8 & 52.7 & 42.4 & 49.6 & 48.2 & 55.7 & 15.2 & 44 & 47.3 & 73.7 \\
\hline
\end{tabular}

$\%$ yield $\mathbf{3 0}$

\begin{tabular}{|rrrrrrrrrrrr|}
\hline 0 & 0 & 0 & 0 & 0 & 0 & 0 & 0 & 0 & 0 & 0 & 0 \\
0 & 0 & 5.94 & 0 & 0 & 0 & 0 & 0 & 0 & 0 & 0 & 0 \\
0 & 7.31 & 0 & 0 & 0 & 0 & 0 & 0 & 0 & 0 & 0 & 0 \\
0 & 0 & 9.64 & 6.13 & 0 & 0 & 0 & 0 & 0 & 0 & 0 & 0 \\
\hline 0 & 0 & 0 & 0 & 0 & 0 & 0 & 0 & 0 & 0 & 0 & 0 \\
0 & 0 & 0 & 0 & 12.3 & 0 & 0 & 0 & 0 & 0 & 0 & 0 \\
0 & 0 & 0 & 0 & 0 & 8.67 & 7.45 & 10.1 & 7.52 & 0 & 0 & 5.77 \\
5.83 & 0 & 9.91 & 0 & 0 & 0 & 5.07 & 7.82 & 10.4 & 0 & 4.11 & 3.3 \\
\hline
\end{tabular}

Yield and conversions determined by UPLC/MS ditbubiphenyl as internal standard. No 28 was detected in any case. Reactions set up according to general procedure for Mizoroki-Heck screening A. 
Table S3: Mizoroki-Heck Optimization: $\mathrm{Pd}(\mathrm{OAc})_{2}$ Selected Ligands

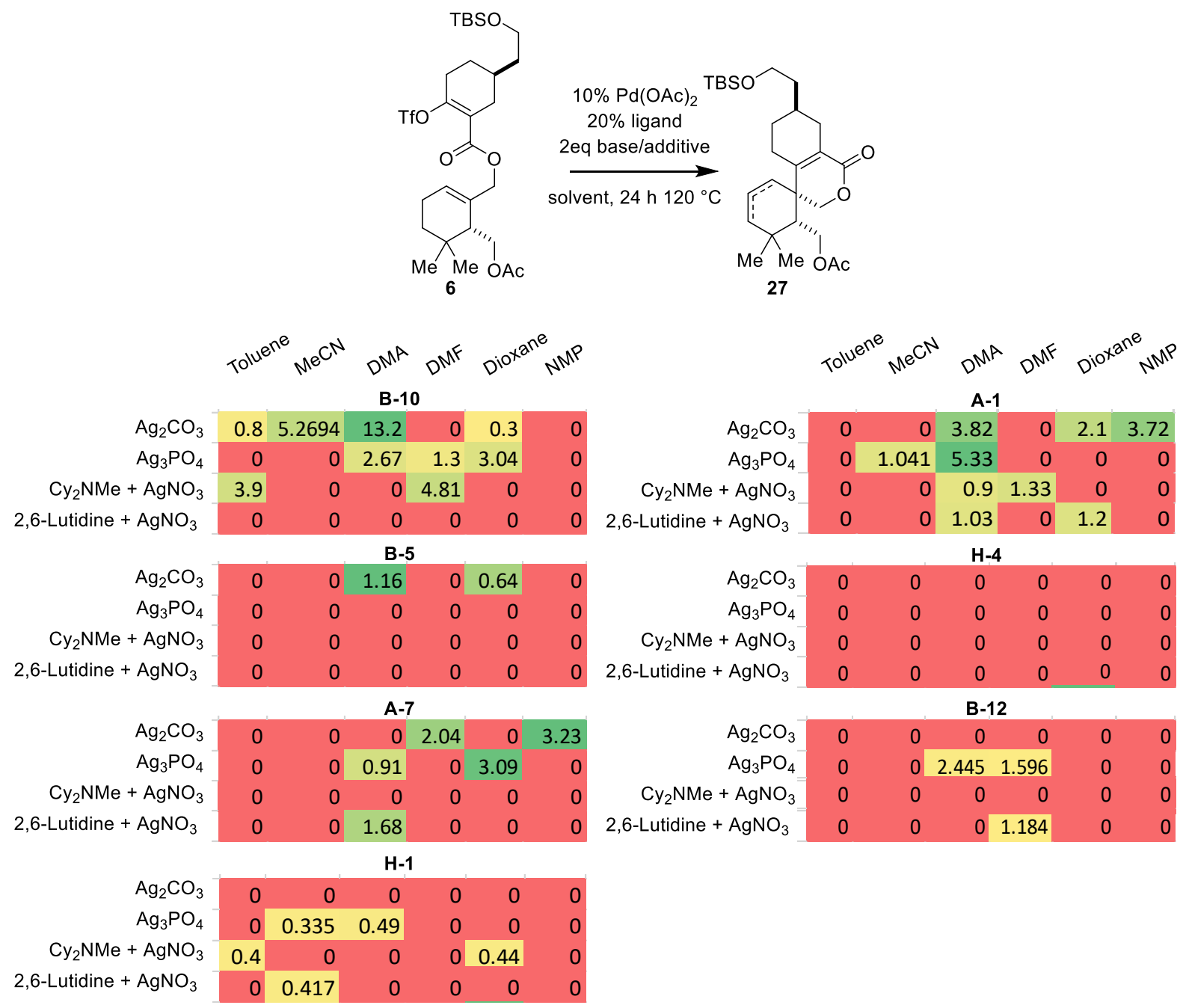

\% Yield as determined by UPLC/MS, 4,4'-di-tert-butylbiphenyl as internal standard. Reactions set up according to general procedure for
Mizoroki-Heck screening B.

Note: attempts to perform these reactions on larger scale to isolate product from those reactions that afforded $>4 \%$ yield in this assay proved to be unsuccessful and no desired product was obtained when using ligand B-10 with $\mathrm{Ag}_{2} \mathrm{CO}_{3}$ in DMA or when using ligand A-1 in DMA with $\mathrm{Ag}_{3} \mathrm{PO}_{4}$ as base. Using A-1 with $\mathrm{Ag}_{2} \mathrm{CO}_{3}$ in DMA was therefore the most encouraging result and is included in manuscript Table 1 , entry 4. 
Table S4: Mizoroki-Heck Optimization: [(allyl)PdCl $]_{2}$ Ligand evaluation with $\mathrm{Ag}_{2} \mathrm{CO}_{3}$ (Manuscript Table 2, entry 1)

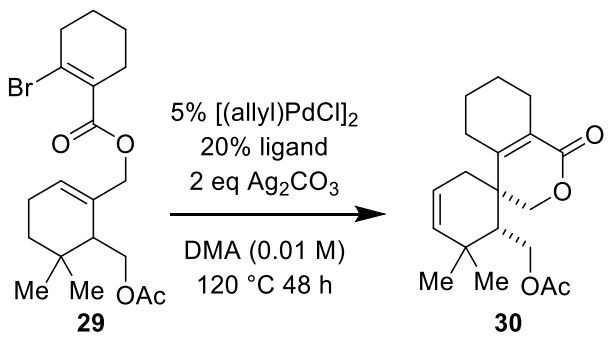

\begin{tabular}{|c|c|c|c|c|c|}
\hline $\mathrm{Pd}\left(\mathrm{PPh}_{3}\right)_{4}{ }^{\mathrm{a}}$ & $\mathrm{PPh}_{3}$ & $\mathrm{PCy}_{3}$ & $\mathrm{tBu}_{3} \mathrm{P}$ & $\mathrm{H}-9$ & H-5 \\
\hline $\mathrm{dppm}$ & $\mathrm{dppp}$ & $\mathrm{B}-3$ & $\mathrm{~B}-4$ & $\mathrm{~B}-6$ & BIPHEP \\
\hline A-2 & A-1 & B-10 & A-6 & A-8 & B-12 \\
\hline S-1 & A-9 & S-2 & S-3 & H-1 & H-16 \\
\hline
\end{tabular}

\% Conversion
\begin{tabular}{|cccccc|}
\hline 10.0 & 13.3 & 23.6 & 28.0 & 64.2 & 68.9 \\
24.6 & 28.9 & 30.2 & 33.6 & 67.4 & 62.2 \\
30.1 & 32.3 & 35.4 & 29.6 & 27.8 & 51.2 \\
22.5 & 44.1 & 53.6 & 27.3 & 44.7 & 70.2 \\
\hline
\end{tabular}

$\%$ yield 32

\begin{tabular}{|llllll|}
\hline 5.3 & 0.0 & 0.0 & 0.0 & 12.0 & 4.3 \\
0.0 & 0.0 & 0.0 & 0.0 & 0.0 & 3.1 \\
0.0 & 0.0 & 0.0 & 0.0 & 0.0 & 6.5 \\
0.0 & 0.0 & 0.0 & 5.9 & 0.5 & 0.5 \\
\hline
\end{tabular}

Yield and conversions determined by GC/FID using dodecane as internal standard. Reactions set up according to general procedure for Mizoroki-Heck screening $B$. a) No $\mathrm{Pd}$ dimer added to this reaction. 
Table S5: Mizoroki-Heck Optimization: [(allyl)PdCl $]_{2}$ Ligand evaluation with $\mathrm{Cy}_{2} \mathrm{NMe}$ and $\mathrm{AgSbF}_{6}$ (Manuscript Table 2, entry 2)

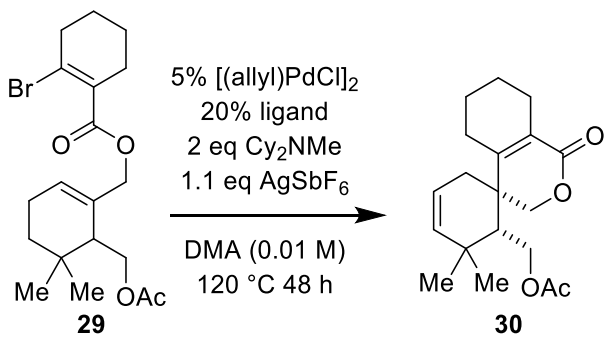

\begin{tabular}{|c|c|c|c|c|c|}
\hline $\mathrm{Pd}\left(\mathrm{PPh}_{3}\right)_{4}{ }^{\mathrm{a}}$ & $\mathrm{PPh}_{3}$ & $\mathrm{PC}_{3}$ & $\mathrm{tBu}_{3} \mathrm{P}$ & $\mathrm{H}-9$ & $\mathrm{H}-5$ \\
\hline $\mathrm{dppm}$ & $\mathrm{dppp}$ & $\mathrm{B}-3$ & $\mathrm{~B}-4$ & $\mathrm{~B}-6$ & BIPHEP \\
\hline $\mathrm{A}-2$ & $\mathrm{~A}-1$ & $\mathrm{~B}-10$ & $\mathrm{~A}-6$ & $\mathrm{~A}-8$ & $\mathrm{~B}-12$ \\
\hline $\mathrm{S}-1$ & $\mathrm{~A}-9$ & $\mathrm{~S}-2$ & $\mathrm{~S}-3$ & $\mathrm{H}-1$ & $\mathrm{H}-16$ \\
\hline
\end{tabular}

\begin{tabular}{|c|c|c|c|c|c|}
\hline 7.7 & 74.4 & 62.8 & 79.5 & 100.0 & 100.0 \\
\hline 51.2 & 34.6 & 53.1 & 71.7 & 100.0 & 61.7 \\
\hline 40.1 & 64.3 & 62.0 & 32.7 & 82.8 & 89.3 \\
\hline 3.3 & 38.7 & 43.2 & 0.0 & 96.1 & 68.7 \\
\hline \multicolumn{6}{|c|}{$\%$ yield 32} \\
\hline 1.1 & 3.1 & 1.2 & 2.5 & 4.2 & 7.4 \\
\hline 0.0 & 0.0 & 0.0 & 0.0 & 0.0 & 0.0 \\
\hline 0.9 & 5.7 & 2.0 & 1.0 & 5.8 & 12.4 \\
\hline 0.0 & 2.8 & 0.0 & 0.0 & 9.1 & 9.1 \\
\hline
\end{tabular}

Yield and conversions determined by GC/FID using dodecane as internal standard. Reactions set up according to general procedure for Mizoroki-Heck screening B. a) No $\mathrm{Pd}$ dimer added to this reaction. 
Table S6: Mizoroki-Heck Optimization: $[(\text { allyl }) \mathrm{PdCl}]_{2}$ Selected Ligands with $\mathrm{Cy}_{2} \mathrm{NMe}$ and Various Silver Salts (Manuscript Table 2, entry 3)

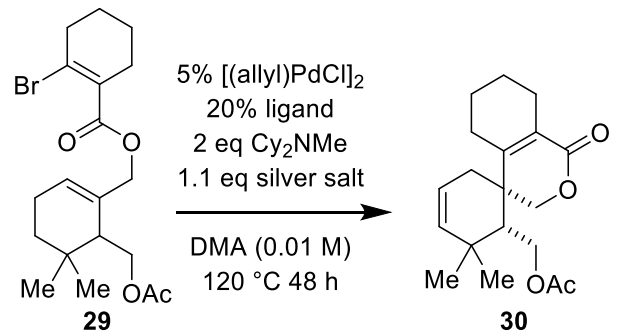

\begin{tabular}{|c|c|c|c|c|c|}
\multicolumn{1}{|c}{ AgOTs } & AgTFA & AgBF $_{4}$ & AgSbF $_{6}$ & AgPF $_{6}$ & AgOTf \\
\hline H-5 & H-5 & H-5 & H-5 & H-5 & H-5 \\
\hline H-9 & H-9 & H-9 & H-9 & H-9 & H-9 \\
\hline B-12 & B-12 & B-12 & B-12 & B-12 & B-12 \\
\hline H-1 & H-1 & H-1 & H-1 & H-1 & H-1 \\
\hline
\end{tabular}

\% conversion
\begin{tabular}{|c|c|c|c|c|c|}
\hline 98.4 & 99.2 & 100 & 99.6 & 97.7 & 99.7 \\
99.6 & 99.8 & 99.9 & 100 & 100 & 100 \\
91.8 & 85.7 & 98.4 & 91.6 & 80.1 & 90.6 \\
96.2 & 97.5 & 99.2 & 98.8 & 99.4 & 99.6 \\
\hline
\end{tabular}

$\%$ yield 32

\begin{tabular}{|c|c|c|c|c|c|}
\hline 14.7 & 9.8 & 9.7 & 16.4 & 15.2 & 15.1 \\
18.1 & 21.3 & 9.3 & 14.7 & 15.8 & 13.9 \\
13.2 & 6.8 & 11.8 & 12.3 & 8.3 & 10.8 \\
19.6 & 12.3 & 11.4 & 16.7 & 19.3 & 17.1 \\
\hline
\end{tabular}

Yield and conversions determined by GC/FID using dodecane as internal standard. Reactions set up according to general procedure for Mizoroki-Heck screening $B$. 
Table S7: Mizoroki-Heck Optimization: [(allyl)PdCl $]_{2}$ Selected Ligands with AgOTs and Varied Organic Bases (Manuscript Table 2, entry 4)

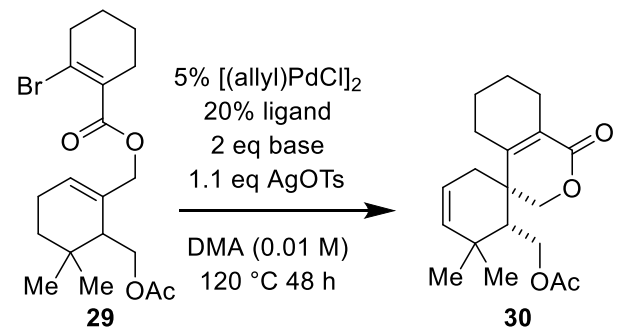

\begin{tabular}{|c|c|c|c|c|c|}
\hline H-5 & H-5 & H-5 & H-5 & H-5 & $\mathrm{H}-5$ \\
\hline H-9 & H-9 & H-9 & H-9 & H-9 & H-9 \\
\hline B-12 & B-12 & B-12 & B-12 & B-12 & B-12 \\
\hline $\mathrm{H}-1$ & H-1 & H-1 & $\mathrm{H}-1$ & $\mathrm{H}-1$ & $\mathrm{H}-1$ \\
\hline
\end{tabular}

\% conversion
\begin{tabular}{|c|c|c|c|c|c|}
\hline 100.0 & 70.0 & 90.4 & 28.5 & 90.3 & 78.4 \\
100.0 & 97.2 & 99.0 & 78.5 & 81.4 & 98.1 \\
86.0 & 50.9 & 63.4 & 16.4 & 98.5 & 79.7 \\
98.5 & 77.3 & 74.6 & 56.6 & 89.5 & 81.8 \\
\hline \% yield 32 & \multicolumn{5}{|l|}{} \\
\hline 1.5 & 27.1 & 23.5 & 4.9 & 0.0 & 44.9 \\
\hline 32.0 & 50.6 & 35.8 & 19.5 & 0.0 & 68.4 \\
25.4 & 26.5 & 16.4 & 2.3 & 0.0 & 47.7 \\
31.1 & 41.1 & 24.6 & 12.1 & 0.0 & 52.3 \\
\hline
\end{tabular}

Yield and conversions determined by GC/FID using dodecane as internal standard. Reactions set up according to general procedure for Mizoroki-Heck screening B. 
Table S8: Mizoroki-Heck Optimization: Concentration of Precatalyst and Silver Salt

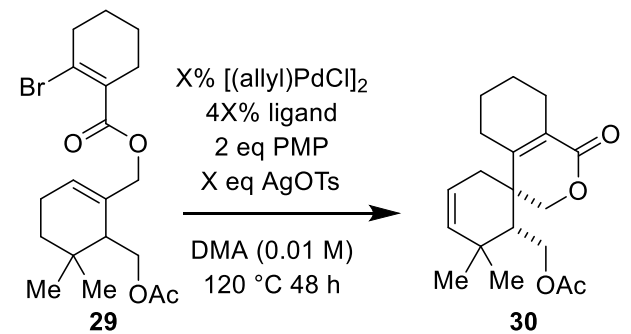

\begin{tabular}{|c|c|c|c|c|c|c|c|c|c|c|c|c|}
\hline \multirow{2}{*}{$\begin{array}{r}\text { catalyst. } \\
\text { loading (eq) }\end{array}$} & \multicolumn{3}{|c|}{0.5 eq AgOTs } & \multicolumn{3}{|c|}{1.1 eq AgOTs } & \multicolumn{3}{|c|}{2 eq AgOTs } & \multicolumn{3}{|c|}{4 eq AgOTs } \\
\hline & 0.025 & 50.05 & 0.1 & 0.025 & 0.05 & 0.1 & 0.025 & 0.05 & 0.1 & 0.025 & 50.05 & 0.1 \\
\hline & H-5 & H-5 & H-5 & H-5 & H-5 & H-5 & H-5 & H-5 & H-5 & H-5 & H-5 & H-5 \\
\hline & H-9 & H-9 & H-9 & H-9 & H-9 & H-9 & H-9 & H-9 & H-9 & H-9 & H-9 & H-9 \\
\hline & B-12 & B-12 & B-12 & B-12 & B-12 & B-12 & B-12 & B-12 & B-12 & B-12 & B-12 & B-12 \\
\hline & $\mathrm{H}-1$ & $\mathrm{H}-1$ & H-1 & $H-1$ & $H-1$ & $H-1$ & $\mathrm{H}-1$ & H-1 & $\mathrm{H}-1$ & $\mathrm{H}-1$ & $H-1$ & $H-1$ \\
\hline
\end{tabular}

\begin{tabular}{|c|c|c|c|c|c|c|c|c|c|c|c|}
\hline 68.1 & 82.3 & 92.3 & 70.0 & 94.2 & 99.1 & 74.1 & 100.0 & 100.0 & 68.4 & 81.6 & 100.0 \\
\hline 65.5 & 86.4 & 99.5 & 70.7 & 98.8 & 100.0 & 79.4 & 100.0 & 100.0 & 37.1 & 100.0 & 100.0 \\
\hline 69.9 & 95.5 & 100.0 & 83.7 & 100.0 & 100.0 & 79.4 & 100.0 & 100.0 & 100.0 & 100.0 & 100.0 \\
\hline 76.7 & 97.5 & 100.0 & 81.7 & 100.0 & 100.0 & 90.9 & 100.0 & 100.0 & 98.8 & 100.0 & 100.0 \\
\hline
\end{tabular}

$\%$ yield 32

\begin{tabular}{|l|l|l|l|l|l|l|l|l|lll|}
\hline 43.5 & 35.5 & 34.9 & 38.7 & 51.5 & 47.2 & 48.1 & 81.7 & 58.1 & 49.1 & 88.7 & 73.9 \\
\hline 25.3 & 32.3 & 33.6 & 29.4 & 46.2 & 38.4 & 41.8 & 51.5 & 43.0 & 25.9 & 55.9 & 52.4 \\
25.4 & 33.5 & 30.2 & 26.8 & 37.2 & 31.1 & 33.0 & 43.2 & 36.6 & 37.4 & 50.9 & 44.2 \\
23.3 & 28.7 & 26.1 & 23.9 & 34.7 & 29.4 & 33.1 & 38.1 & 32.5 & 39.5 & 48.5 & 41.5 \\
\hline
\end{tabular}

Yield and conversions determined by GC/FID using dodecane as internal standard. Reactions set up according to general procedure for Mizoroki-Heck screening B. 
Table S9: Mizoroki-Heck Optimization: Concentration of Silver Salt

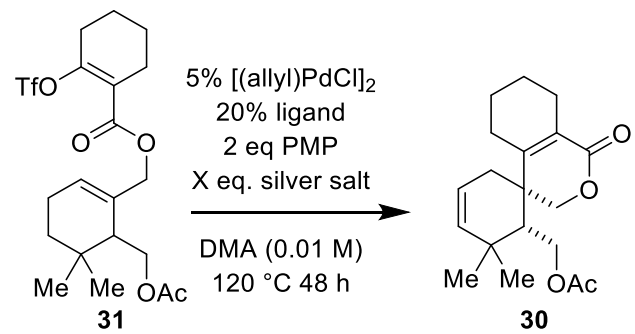

\begin{tabular}{|c|c|c|c|c|c|c|}
\hline \multirow{2}{*}{$\begin{array}{c}\text { silver salt } \\
\text { loading (eq.) }\end{array}$} & \multicolumn{3}{|c|}{ AgOTs } & \multicolumn{3}{|c|}{ AgOTf } \\
\hline & 0 & 1.1 & 4 & 0 & 1.1 & 4 \\
\hline & $\mathrm{H}-5$ & $\mathrm{H}-5$ & $H-5$ & $\mathrm{H}-5$ & $\mathrm{H}-5$ & H-5 \\
\hline & B-12 & B-12 & B-12 & B-12 & B-12 & B-12 \\
\hline & $\mathrm{H}-1$ & $\mathrm{H}-1$ & $\mathrm{H}-1$ & $\mathrm{H}-1$ & $\mathrm{H}-1$ & $H-1$ \\
\hline & H-9 & H-9 & H-9 & H-9 & $\mathrm{H}-9$ & H-9 \\
\hline & yield 3 & & & & & \\
\hline & 0.2 & 9.6 & 3.2 & 0.3 & 20.8 & 10.9 \\
\hline & 0.3 & 6.2 & 0.0 & 0.6 & 19.5 & 5.4 \\
\hline & 0.1 & 15.0 & 1.8 & 0.1 & 16.4 & 1.3 \\
\hline & 0.1 & 27.6 & 29.7 & 0.0 & 26.2 & 25.3 \\
\hline
\end{tabular}

Yield and conversions determined by GC/FID using dodecane as internal standard.Reactions set up according to general procedure for Mizoroki-Heck screening B. 
Table S10: Mizoroki-Heck Optimization: Reaction Concentration and Concentration of Silver Salt
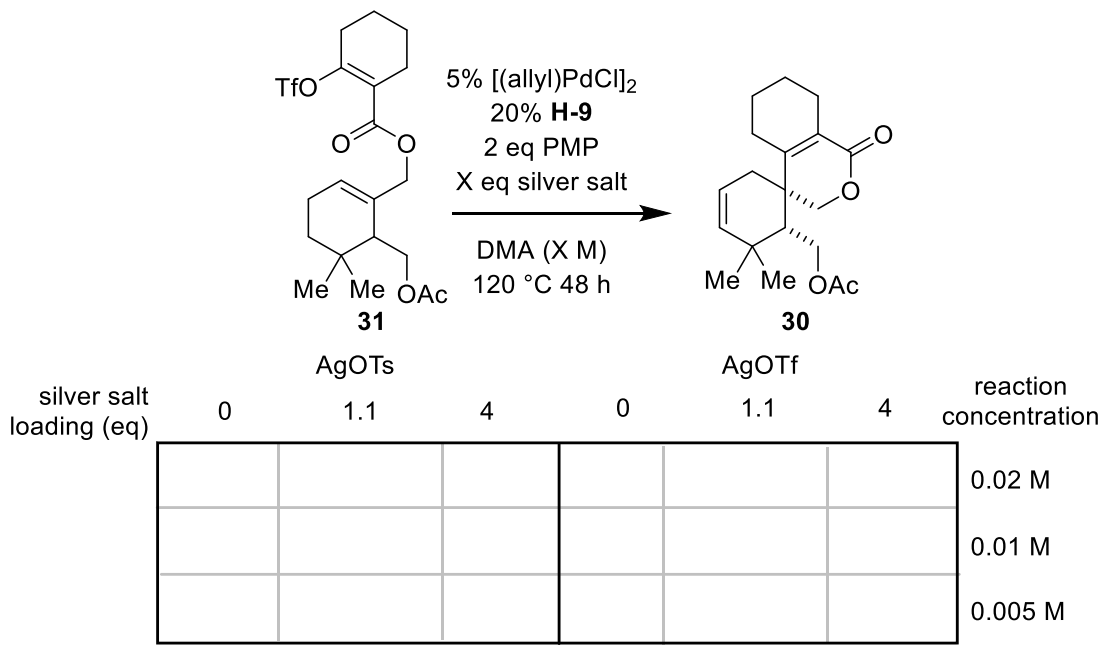

\% yield 32
\begin{tabular}{|c|c|c|c|c|c|}
\hline 0.3 & 34.3 & 0.0 & 0.0 & 37.7 & 22.2 \\
0.2 & 43.6 & 24.1 & 0.0 & 43.1 & 28.3 \\
0.0 & 40.3 & 11.4 & 0.0 & 53.4 & 43.2 \\
\hline
\end{tabular}

Yield and conversions determined by GC/FID using dodecane as internal standard.Reactions set up according to general procedure for Mizoroki-Heck screening $B$. 
Table S11: Mizoroki-Heck Optimization: Concentration of Silver Salt

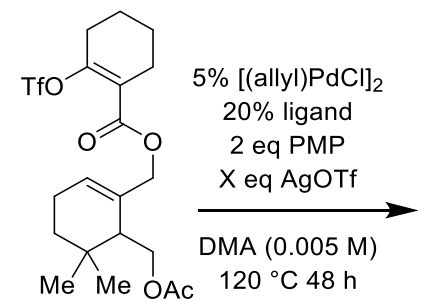

31

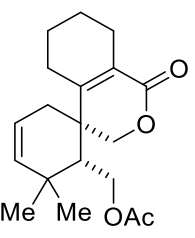

30

\begin{tabular}{lcccccc|}
\multicolumn{3}{c}{ AgOTs } & \multicolumn{3}{c}{ AgOTf } \\
\multirow{2}{*}{$\begin{array}{c}\text { eq. } \mathrm{Ag} \\
\text { salt }\end{array}$} & 0.05 & 0.1 & 0.5 & 1 & 2 & 3 \\
\cline { 2 - 7 } & H-9 & H-9 & H-9 & H-9 & H-9 & H-9 \\
\hline
\end{tabular}

$\%$ yield 32

\begin{tabular}{|l|l|l|l|l|l|}
\hline 0.5 & 0.5 & 0.0 & 0.0 & 47.4 & 51.6 \\
\hline
\end{tabular}

Yield and conversions determined by GC/FID using dodecane as internal standard. Reactions set up according to general procedure for Mizoroki-Heck screening $B$. 


\section{X-Ray Crystallographic Data}

\section{Structure Determination of para-bromobenzoate derivative of 19.}

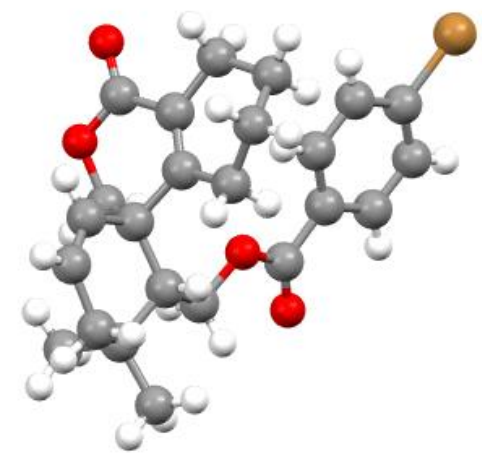

\section{(CCDC 1996075)}

Colorless blocks of para-bromobenzoate derivative of $\mathbf{1 9}$ were grown from an acetonitrile solution of the compound at $22 \mathrm{deg}$. C. A crystal of dimensions $0.15 \times 0.14 \times 0.09 \mathrm{~mm}$ was mounted on a Rigaku AFC10K Saturn 944+ CCD-based X-ray diffractometer equipped with a low temperature device and Micromax-007HF Cu-target micro-focus rotating anode $(\lambda=1.54187 \mathrm{~A})$ operated at $1.2 \mathrm{~kW}$ power $(40 \mathrm{kV}, 30 \mathrm{~mA})$. The X-ray intensities were measured at $85(1) \mathrm{K}$ with the detector placed at a distance $42.00 \mathrm{~mm}$ from the crystal. A total of 2028 images were collected with an oscillation width of $1.0^{\circ}$ in $\mathrm{w}$. The exposure times were $1 \mathrm{sec}$. for the low angle images, 4 sec. for high angle. Rigaku d*trek images were exported to CrysAlisPro for processing and corrected for absorption. The integration of the data yielded a total of 30622 reflections to a maximum $2 \mathrm{q}$ value of $138.36^{\circ}$ of which 3744 were independent and 3731 were greater than $2 \sigma(\mathrm{I})$. The final cell constants (Table 1) were based on the xyz centroids 23539 reflections above $10 \sigma(\mathrm{I})$. Analysis of the data showed negligible decay during data collection. The structure was solved and refined with the Bruker SHELXTL (version 2016/6) software package, using the space group $\mathrm{P} 2(1) / c$ with $\mathrm{Z}=4$ for the formula $\mathrm{C}_{2} 4 \mathrm{H}_{2} 7 \mathrm{O} 4 \mathrm{Br}$. All non-hydrogen atoms were refined anisotropically with the hydrogen atoms placed in idealized positions. Full matrix least-squares refinement based on $\mathrm{F}^{2}$ converged at R1 $=0.0292$ and $\mathrm{wR} 2=0.0772$ [based on $\mathrm{I}>2$ sigma(I)], R1 $=0.0294$ and $\mathrm{wR} 2=0.0774$ for all data. Additional details are presented in Table 1 and are given as Supporting Information in a CIF file. Acknowledgement is made for funding from NSF grant CHE-0840456 for X-ray instrumentation.

G.M. Sheldrick (2015) "Crystal structure refinement with SHELXL", Acta Cryst., C71, 3-8 (Open Access).

CrystalClear Expert 2.0 r16, Rigaku Americas and Rigaku Corporation (2014), Rigaku Americas, 9009, TX, USA 77381-5209, Rigaku Tokyo, 196-8666, Japan.

CrysAlisPro 1.171.38.41 (Rigaku Oxford Diffraction, 2015). 


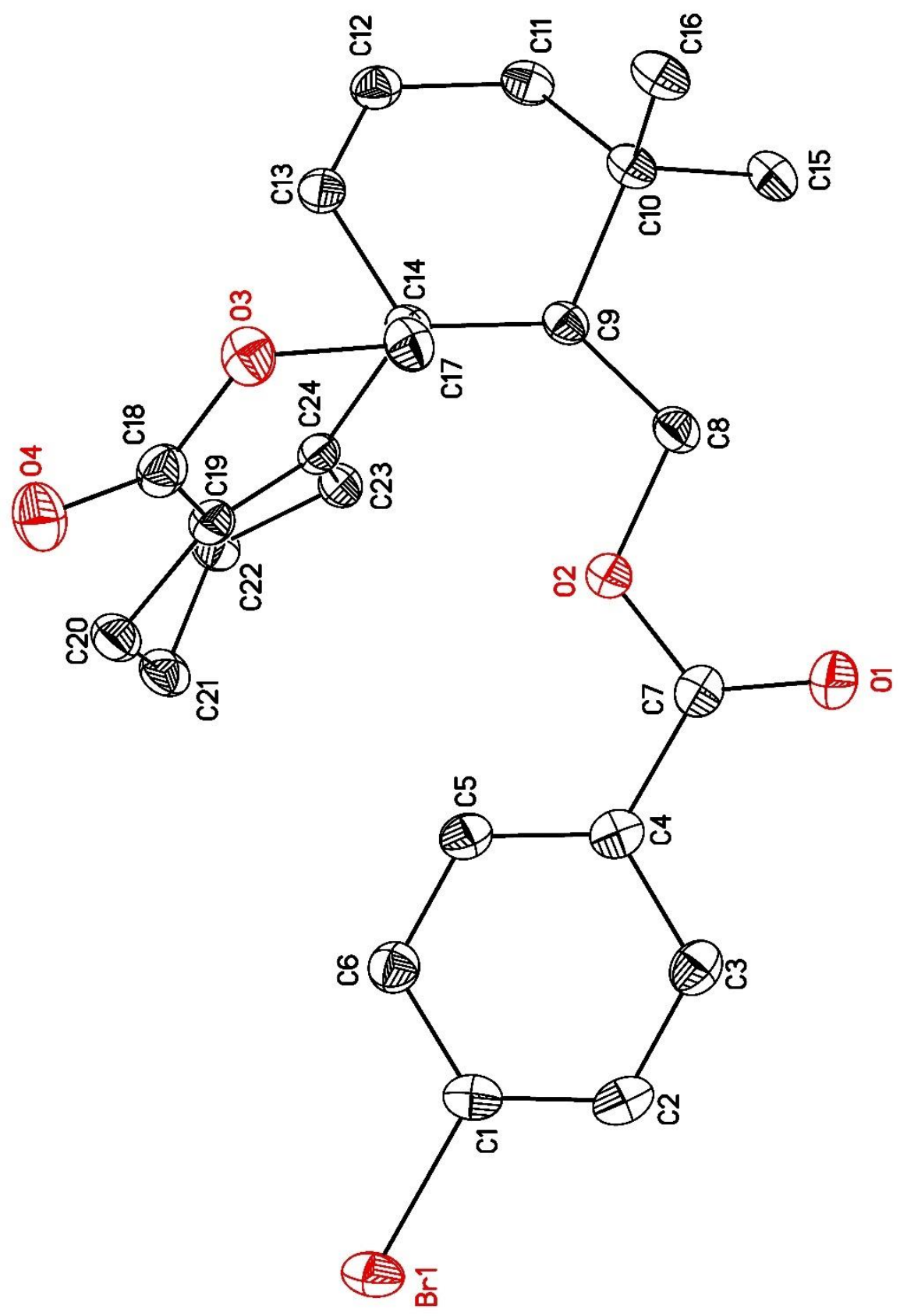

Figure S7. X-ray crystal structure thermal ellipsoid plot at the 50\% probability level. 
Table S12. Crystal data and structure refinement.

Identification code $\quad \mathbf{2 0}$

Empirical formula $\quad \mathrm{C} 24 \mathrm{H} 27 \mathrm{Br} \mathrm{O} 4$

Formula weight $\quad 459.36$

Temperature $\quad 85(2) \mathrm{K}$

Wavelength $\quad 1.54184 \mathrm{~A}$

Crystal system, space group Monoclinic, P2(1)/c

Unit cell dimensions $\quad \mathrm{a}=10.79064(8) \mathrm{A} \quad$ alpha $=90 \mathrm{deg}$.

$\mathrm{b}=25.34034(17) \mathrm{A} \quad$ beta $=111.0121(9) \mathrm{deg}$.

$\mathrm{c}=7.96505(7) \mathrm{A}$ gamma $=90 \mathrm{deg}$.

Volume 2033.13(3) $\mathrm{A}^{\wedge} 3$

Z, Calculated density $\quad 4,1.501 \mathrm{Mg} / \mathrm{m}^{\wedge} 3$

Absorption coefficient $2.989 \mathrm{~mm}^{\wedge}-1$

$\mathrm{F}(000) \quad 952$

Crystal size $\quad 0.150 \times 0.140 \times 0.090 \mathrm{~mm}$

Theta range for data collection 4.389 to $69.182 \mathrm{deg}$.

Limiting indices $\quad-13<=\mathrm{h}<=13,-30<=\mathrm{k}<=30,-8<=\mathrm{l}<=9$

Reflections collected / unique 30622 / 3744 [R(int) $=0.0397]$

Completeness to theta $=67.684 \quad 99.3 \%$

Absorption correction Semi-empirical from equivalents

Max. and min. transmission $\quad 1.00000$ and 0.71503

Refinement method Full-matrix least-squares on $\mathrm{F}^{\wedge} 2$

Data / restraints / parameters 3744 / 0 / 265

Goodness-of-fit on $\mathrm{F}^{\wedge} 2 \quad 1.108$

Final R indices $[\mathrm{I}>2 \operatorname{sigma}(\mathrm{I})] \quad \mathrm{R} 1=0.0292, \mathrm{wR} 2=0.0772$

$\mathrm{R}$ indices (all data) $\quad \mathrm{R} 1=0.0294, \mathrm{wR} 2=0.0774$ 
Extinction coefficient $\quad 0.00143(18)$

Largest diff. peak and hole $\quad 0.525$ and -0.655 e. $\mathrm{A}^{\wedge}-3$ 
Table S13. Atomic coordinates ( x 10^4) and equivalent isotropic displacement parameters $\left(\mathrm{A}^{\wedge} 2 \times 10^{\wedge} 3\right)$.

$\mathrm{U}(\mathrm{eq})$ is defined as one third of the trace of the orthogonalized Uij tensor.

\begin{tabular}{lcccc}
\hline \multicolumn{5}{c}{} \\
$\mathrm{x}$ & $\mathrm{y}$ & $\mathrm{U}(\mathrm{eq})$ & \\
& & & \\
$\mathrm{B}(1)$ & $8787(1)$ & $7887(1)$ & $11711(1)$ & $26(1)$ \\
$\mathrm{O}(1)$ & $2572(1)$ & $7832(1)$ & $5046(2)$ & $29(1)$ \\
$\mathrm{O}(2)$ & $3032(1)$ & $6970(1)$ & $5625(2)$ & $19(1)$ \\
$\mathrm{O}(3)$ & $2007(1)$ & $5817(1)$ & $8719(2)$ & $21(1)$ \\
$\mathrm{O}(4)$ & $3755(1)$ & $5631(1)$ & $11115(2)$ & $27(1)$ \\
$\mathrm{C}(1)$ & $7097(2)$ & $7766(1)$ & $9909(2)$ & $21(1)$ \\
$\mathrm{C}(2)$ & $6345(2)$ & $8188(1)$ & $8981(3)$ & $24(1)$ \\
$\mathrm{C}(3)$ & $5110(2)$ & $8092(1)$ & $7684(3)$ & $23(1)$ \\
$\mathrm{C}(4)$ & $4632(2)$ & $7577(1)$ & $7316(2)$ & $19(1)$ \\
$\mathrm{C}(5)$ & $5400(2)$ & $7160(1)$ & $8275(3)$ & $21(1)$ \\
$\mathrm{C}(6)$ & $6639(2)$ & $7251(1)$ & $9577(2)$ & $23(1)$ \\
$\mathrm{C}(7)$ & $3308(2)$ & $7486(1)$ & $5885(2)$ & $20(1)$ \\
$\mathrm{C}(8)$ & $1742(2)$ & $6836(1)$ & $4299(2)$ & $20(1)$ \\
$\mathrm{C}(9)$ & $1721(2)$ & $6244(1)$ & $3958(2)$ & $17(1)$ \\
$\mathrm{C}(10)$ & $388(2)$ & $6073(1)$ & $2477(2)$ & $18(1)$ \\
$\mathrm{C}(11)$ & $551(2)$ & $5497(1)$ & $1981(2)$ & $21(1)$ \\
$\mathrm{C}(12)$ & $1054(2)$ & $5148(1)$ & $3597(2)$ & $21(1)$ \\
$\mathrm{C}(13)$ & $1707(2)$ & $5326(1)$ & $5248(2)$ & $19(1)$ \\
$\mathrm{C}(14)$ & $2131(2)$ & $5898(1)$ & $5703(2)$ & $17(1)$ \\
$\mathrm{C}(15)$ & $116(2)$ & $6409(1)$ & $780(2)$ & $23(1)$ \\
$\mathrm{C}(16)$ & $-822(2)$ & $6115(1)$ & $3061(2)$ & $22(1)$ \\
$\mathrm{C}(17)$ & $1577(2)$ & $6122(1)$ & $7073(2)$ & $19(1)$ \\
$\mathrm{C}(18)$ & $3330(2)$ & $5757(1)$ & $9542(2)$ & $21(1)$ \\
$\mathrm{C}(19)$ & $4178(2)$ & $5814(1)$ & $8451(2)$ & $18(1)$ \\
$\mathrm{C}(20)$ & $5647(2)$ & $5740(1)$ & $9494(2)$ & $23(1)$ \\
$\mathrm{C}(21)$ & $6499(2)$ & $5779(1)$ & $8323(2)$ & $24(1)$ \\
$\mathrm{C}(22)$ & $5789(2)$ & $5531(1)$ & $6493(2)$ & $23(1)$ \\
$\mathrm{C}(23)$ & $4488(2)$ & $5819(1)$ & $5518(2)$ & $19(1)$ \\
$\mathrm{C}(24)$ & $3641(2)$ & $5861(1)$ & $6653(2)$ & $16(1)$ \\
& & & & \\
\hline & & &
\end{tabular}


Table S14. Bond lengths [A] and angles [deg].

\begin{tabular}{|c|c|}
\hline $\operatorname{Br}(1)-C(1)$ & $1.8988(18)$ \\
\hline $\mathrm{O}(1)-\mathrm{C}(7)$ & $1.212(2)$ \\
\hline $\mathrm{O}(2)-\mathrm{C}(7)$ & $1.340(2)$ \\
\hline $\mathrm{O}(2)-\mathrm{C}(8)$ & $1.453(2)$ \\
\hline $\mathrm{O}(3)-\mathrm{C}(18)$ & $1.349(2)$ \\
\hline $\mathrm{O}(3)-\mathrm{C}(17)$ & $1.447(2)$ \\
\hline $\mathrm{O}(4)-\mathrm{C}(18)$ & $1.211(2)$ \\
\hline$C(1)-C(6)$ & $1.386(3)$ \\
\hline $\mathrm{C}(1)-\mathrm{C}(2)$ & $1.386(3)$ \\
\hline $\mathrm{C}(2)-\mathrm{C}(3)$ & $1.384(3)$ \\
\hline $\mathrm{C}(2)-\mathrm{H}(2)$ & 0.9500 \\
\hline$C(3)-C(4)$ & $1.395(2)$ \\
\hline $\mathrm{C}(3)-\mathrm{H}(3)$ & 0.9500 \\
\hline$C(4)-C(5)$ & $1.390(3)$ \\
\hline$C(4)-C(7)$ & $1.492(2)$ \\
\hline $\mathrm{C}(5)-\mathrm{C}(6)$ & $1.386(3)$ \\
\hline $\mathrm{C}(5)-\mathrm{H}(5)$ & 0.9500 \\
\hline $\mathrm{C}(6)-\mathrm{H}(6)$ & 0.9500 \\
\hline $\mathrm{C}(8)-\mathrm{C}(9)$ & $1.525(2)$ \\
\hline $\mathrm{C}(8)-\mathrm{H}(8 \mathrm{~A})$ & 0.9900 \\
\hline $\mathrm{C}(8)-\mathrm{H}(8 \mathrm{~B})$ & 0.9900 \\
\hline C(9)-C(10) & $1.559(2)$ \\
\hline C(9)-C(14) & $1.567(2)$ \\
\hline $\mathrm{C}(9)-\mathrm{H}(9)$ & 1.0000 \\
\hline$C(10)-C(15)$ & $1.534(2)$ \\
\hline$C(10)-C(16)$ & $1.538(2)$ \\
\hline $\mathrm{C}(10)-\mathrm{C}(11)$ & $1.540(2)$ \\
\hline $\mathrm{C}(11)-\mathrm{C}(12)$ & $1.494(2)$ \\
\hline $\mathrm{C}(11)-\mathrm{H}(11 \mathrm{~A})$ & 0.9900 \\
\hline $\mathrm{C}(11)-\mathrm{H}(11 \mathrm{~B})$ & 0.9900 \\
\hline $\mathrm{C}(12)-\mathrm{C}(13)$ & $1.328(2)$ \\
\hline $\mathrm{C}(12)-\mathrm{H}(12)$ & 0.9500 \\
\hline $\mathrm{C}(13)-\mathrm{C}(14)$ & $1.523(2)$ \\
\hline $\mathrm{C}(13)-\mathrm{H}(13)$ & 0.9500 \\
\hline $\mathrm{C}(14)-\mathrm{C}(17)$ & $1.529(2)$ \\
\hline $\mathrm{C}(14)-\mathrm{C}(24)$ & $1.534(2)$ \\
\hline $\mathrm{C}(15)-\mathrm{H}(15 \mathrm{~A})$ & 0.9800 \\
\hline $\mathrm{C}(15)-\mathrm{H}(15 \mathrm{~B})$ & 0.9800 \\
\hline $\mathrm{C}(15)-\mathrm{H}(15 \mathrm{C})$ & 0.9800 \\
\hline $\mathrm{C}(16)-\mathrm{H}(16 \mathrm{~A})$ & 0.9800 \\
\hline $\mathrm{C}(16)-\mathrm{H}(16 \mathrm{~B})$ & 0.9800 \\
\hline $\mathrm{C}(16)-\mathrm{H}(16 \mathrm{C})$ & 0.9800 \\
\hline $\mathrm{C}(17)-\mathrm{H}(17 \mathrm{~A})$ & 0.9900 \\
\hline $\mathrm{C}(17)-\mathrm{H}(17 \mathrm{~B})$ & 0.9900 \\
\hline C(18)-C(19) & $1.478(2)$ \\
\hline$C(19)-C(24)$ & $1.344(2)$ \\
\hline C(19)-C(20) & $1.514(2)$ \\
\hline $\mathrm{C}(20)-\mathrm{C}(21)$ & $1.530(3)$ \\
\hline
\end{tabular}




$\begin{array}{lc} & \\ \mathrm{C}(20)-\mathrm{H}(20 \mathrm{~A}) & 0.9900 \\ \mathrm{C}(20)-\mathrm{H}(20 \mathrm{~B}) & 0.9900 \\ \mathrm{C}(21)-\mathrm{C}(22) & 1.518(2) \\ \mathrm{C}(21)-\mathrm{H}(21 \mathrm{~A}) & 0.9900 \\ \mathrm{C}(21)-\mathrm{H}(21 \mathrm{~B}) & 0.9900 \\ \mathrm{C}(22)-\mathrm{C}(23) & 1.524(2) \\ \mathrm{C}(22)-\mathrm{H}(22 \mathrm{~A}) & 0.9900 \\ \mathrm{C}(22)-\mathrm{H}(22 \mathrm{~B}) & 0.9900 \\ \mathrm{C}(23)-\mathrm{C}(24) & 1.502(2) \\ \mathrm{C}(23)-\mathrm{H}(23 \mathrm{~A}) & 0.9900 \\ \mathrm{C}(23)-\mathrm{H}(23 \mathrm{~B}) & 0.9900 \\ & \\ \mathrm{C}(7)-\mathrm{O}(2)-\mathrm{C}(8) & 116.19(13) \\ \mathrm{C}(18)-\mathrm{O}(3)-\mathrm{C}(17) & 116.11(13) \\ \mathrm{C}(6)-\mathrm{C}(1)-\mathrm{C}(2) & 121.66(17) \\ \mathrm{C}(6)-\mathrm{C}(1)-\mathrm{Br}(1) & 118.50(14) \\ \mathrm{C}(2)-\mathrm{C}(1)-\mathrm{Br}(1) & 119.83(13) \\ \mathrm{C}(3)-\mathrm{C}(2)-\mathrm{C}(1) & 118.95(16) \\ \mathrm{C}(3)-\mathrm{C}(2)-\mathrm{H}(2) & 120.5 \\ \mathrm{C}(1)-\mathrm{C}(2)-\mathrm{H}(2) & 120.5 \\ \mathrm{C}(2)-\mathrm{C}(3)-\mathrm{C}(4) & 120.42(17) \\ \mathrm{C}(2)-\mathrm{C}(3)-\mathrm{H}(3) & 119.8 \\ \mathrm{C}(4)-\mathrm{C}(3)-\mathrm{H}(3) & 119.8 \\ \mathrm{C}(5)-\mathrm{C}(4)-\mathrm{C}(3) & 119.53(16) \\ \mathrm{C}(5)-\mathrm{C}(4)-\mathrm{C}(7) & 121.43(15) \\ \mathrm{C}(3)-\mathrm{C}(4)-\mathrm{C}(7) & 119.04(15) \\ \mathrm{C}(6)-\mathrm{C}(5)-\mathrm{C}(4) & 120.65(16) \\ \mathrm{C}(6)-\mathrm{C}(5)-\mathrm{H}(5) & 119.7 \\ \mathrm{C}(4)-\mathrm{C}(5)-\mathrm{H}(5) & 119.7 \\ \mathrm{C}(5)-\mathrm{C}(6)-\mathrm{C}(1) & 118.78(17) \\ \mathrm{C}(5)-\mathrm{C}(6)-\mathrm{H}(6) & 120.6 \\ \mathrm{C}(1)-\mathrm{C}(6)-\mathrm{H}(6) & 120.6 \\ \mathrm{O}(1)-\mathrm{C}(7)-\mathrm{O}(2) & 123.80(16) \\ \mathrm{O}(1)-\mathrm{C}(7)-\mathrm{C}(4) & 124.62(16) \\ \mathrm{O}(2)-\mathrm{C}(7)-\mathrm{C}(4) & 111.58(14) \\ \mathrm{O}(2)-\mathrm{C}(8)-\mathrm{C}(9) & 107.96(13) \\ \mathrm{O}(2)-\mathrm{C}(8)-\mathrm{H}(8 \mathrm{~A}) & 110.1 \\ \mathrm{C}(9)-\mathrm{C}(8)-\mathrm{H}(8 \mathrm{~A}) & 110.1 \\ \mathrm{O}(2)-\mathrm{C}(8)-\mathrm{H}(8 \mathrm{~B}) & 110.1 \\ \mathrm{C}(9)-\mathrm{C}(8)-\mathrm{H}(8 \mathrm{~B}) & 110.1 \\ \mathrm{H}(8 \mathrm{~A})-\mathrm{C}(8)-\mathrm{H}(8 \mathrm{~B}) & 108.4 \\ \mathrm{C}(8)-\mathrm{C}(9)-\mathrm{C}(10) & 111.05(13) \\ \mathrm{C}(8)-\mathrm{C}(9)-\mathrm{C}(14) & 114.29(13) \\ \mathrm{C}(10)-\mathrm{C}(9)-\mathrm{C}(14) & 113.98(13) \\ \mathrm{C}(8)-\mathrm{C}(9)-\mathrm{H}(9) & 105.5 \\ \mathrm{C}(10)-\mathrm{C}(9)-\mathrm{H}(9) & 105.5 \\ \mathrm{C}(14)-\mathrm{C}(9)-\mathrm{H}(9) & 105.5 \\ \mathrm{C}(15)-\mathrm{C}(10)-\mathrm{C}(16) & 108.27(14) \\ \mathrm{C}(15)-\mathrm{C}(10)-\mathrm{C}(11) & 107.67(14) \\ \mathrm{C}(16)-\mathrm{C}(10)-\mathrm{C}(11) & 109.24(14) \\ \mathrm{C}(15)-\mathrm{C}(10)-\mathrm{C}(9) & 110.23(14) \\ & \end{array}$




$\begin{array}{lc}\mathrm{C}(16)-\mathrm{C}(10)-\mathrm{C}(9) & 113.91(14) \\ \mathrm{C}(11)-\mathrm{C}(10)-\mathrm{C}(9) & 107.34(13) \\ \mathrm{C}(12)-\mathrm{C}(11)-\mathrm{C}(10) & 112.31(14) \\ \mathrm{C}(12)-\mathrm{C}(11)-\mathrm{H}(11 \mathrm{~A}) & 109.1 \\ \mathrm{C}(10)-\mathrm{C}(11)-\mathrm{H}(11 \mathrm{~A}) & 109.1 \\ \mathrm{C}(12)-\mathrm{C}(11)-\mathrm{H}(11 \mathrm{~B}) & 109.1 \\ \mathrm{C}(10)-\mathrm{C}(11)-\mathrm{H}(11 \mathrm{~B}) & 109.1 \\ \mathrm{H}(11 \mathrm{~A})-\mathrm{C}(11)-\mathrm{H}(11 \mathrm{~B}) & 107.9 \\ \mathrm{C}(13)-\mathrm{C}(12)-\mathrm{C}(11) & 123.44(16) \\ \mathrm{C}(13)-\mathrm{C}(12)-\mathrm{H}(12) & 118.3 \\ \mathrm{C}(11)-\mathrm{C}(12)-\mathrm{H}(12) & 118.3 \\ \mathrm{C}(12)-\mathrm{C}(13)-\mathrm{C}(14) & 124.52(16) \\ \mathrm{C}(12)-\mathrm{C}(13)-\mathrm{H}(13) & 117.7 \\ \mathrm{C}(14)-\mathrm{C}(13)-\mathrm{H}(13) & 117.7 \\ \mathrm{C}(13)-\mathrm{C}(14)-\mathrm{C}(17) & 111.18(13) \\ \mathrm{C}(13)-\mathrm{C}(14)-\mathrm{C}(24) & 103.60(13) \\ \mathrm{C}(17)-\mathrm{C}(14)-\mathrm{C}(24) & 106.75(13) \\ \mathrm{C}(13)-\mathrm{C}(14)-\mathrm{C}(9) & 110.84(13) \\ \mathrm{C}(17)-\mathrm{C}(14)-\mathrm{C}(9) & 111.57(13) \\ \mathrm{C}(24)-\mathrm{C}(14)-\mathrm{C}(9) & 112.58(13) \\ \mathrm{C}(10)-\mathrm{C}(15)-\mathrm{H}(15 \mathrm{~A}) & 109.5 \\ \mathrm{C}(10)-\mathrm{C}(15)-\mathrm{H}(15 \mathrm{~B}) & 109.5 \\ \mathrm{H}(15 \mathrm{~A})-\mathrm{C}(15)-\mathrm{H}(15 \mathrm{~B}) & 109.5 \\ \mathrm{C}(10)-\mathrm{C}(15)-\mathrm{H}(15 \mathrm{C}) & 109.5 \\ \mathrm{H}(15 \mathrm{~A})-\mathrm{C}(15)-\mathrm{H}(15 \mathrm{C}) & 109.5 \\ \mathrm{H}(15 \mathrm{~B})-\mathrm{C}(15)-\mathrm{H}(15 \mathrm{C}) & 109.5 \\ \mathrm{C}(10)-\mathrm{C}(16)-\mathrm{H}(16 \mathrm{~A}) & 109.5 \\ \mathrm{C}(10)-\mathrm{C}(16)-\mathrm{H}(16 \mathrm{~B}) & 109.5 \\ \mathrm{H}(16 \mathrm{~A})-\mathrm{C}(16)-\mathrm{H}(16 \mathrm{~B}) & 109.5 \\ \mathrm{C}(10)-\mathrm{C}(16)-\mathrm{H}(16 \mathrm{C}) & 109.5 \\ \mathrm{H}(16 \mathrm{~A})-\mathrm{C}(16)-\mathrm{H}(16 \mathrm{C}) & 109.5 \\ \mathrm{H}(16 \mathrm{~B})-\mathrm{C}(16)-\mathrm{H}(16 \mathrm{C}) & 109.5 \\ \mathrm{O}(3)-\mathrm{C}(17)-\mathrm{C}(14) & 111.61(13) \\ \mathrm{O}(3)-\mathrm{C}(17)-\mathrm{H}(17 \mathrm{~A}) & 109.3 \\ \mathrm{C}(14)-\mathrm{C}(17)-\mathrm{H}(17 \mathrm{~A}) & 109.3 \\ \mathrm{O}(3)-\mathrm{C}(17)-\mathrm{H}(17 \mathrm{~B}) & 109.3 \\ \mathrm{C}(14)-\mathrm{C}(17)-\mathrm{H}(17 \mathrm{~B}) & 109.3 \\ \mathrm{H}(17 \mathrm{~A})-\mathrm{C}(17)-\mathrm{H}(17 \mathrm{~B}) & 108.0 \\ \mathrm{O}(4)-\mathrm{C}(18)-\mathrm{O}(3) & 118.30(16) \\ \mathrm{O}(4)-\mathrm{C}(18)-\mathrm{C}(19) & 123.34(16) \\ \mathrm{O}(3)-\mathrm{C}(18)-\mathrm{C}(19) & 118.25(15) \\ \mathrm{C}(24)-\mathrm{C}(19)-\mathrm{C}(18) & 120.93(15) \\ \mathrm{C}(24)-\mathrm{C}(19)-\mathrm{C}(20) & 124.26(16) \\ \mathrm{C}(18)-\mathrm{C}(19)-\mathrm{C}(20) & 114.31(15) \\ \mathrm{C}(19)-\mathrm{C}(20)-\mathrm{C}(21) & 113.39(15) \\ \mathrm{C}(19)-\mathrm{C}(20)-\mathrm{H}(20 \mathrm{~A}) & 108.9 \\ \mathrm{C}(21)-\mathrm{C}(20)-\mathrm{H}(20 \mathrm{~A}) & 108.9 \\ \mathrm{C}(19)-\mathrm{C}(20)-\mathrm{H}(20 \mathrm{~B}) & 108.9 \\ \mathrm{C}(21)-\mathrm{C}(20)-\mathrm{H}(20 \mathrm{~B}) & 108.9 \\ \mathrm{H}(20 \mathrm{~A})-\mathrm{C}(20)-\mathrm{H}(20 \mathrm{~B}) & 107.7 \\ \mathrm{C}(22)-\mathrm{C}(21)-\mathrm{C}(20) & 110.60(15) \\ & \\ & \end{array}$




$\begin{array}{lc}\mathrm{C}(22)-\mathrm{C}(21)-\mathrm{H}(21 \mathrm{~A}) & 109.5 \\ \mathrm{C}(20)-\mathrm{C}(21)-\mathrm{H}(21 \mathrm{~A}) & 109.5 \\ \mathrm{C}(22)-\mathrm{C}(21)-\mathrm{H}(21 \mathrm{~B}) & 109.5 \\ \mathrm{C}(20)-\mathrm{C}(21)-\mathrm{H}(21 \mathrm{~B}) & 109.5 \\ \mathrm{H}(21 \mathrm{~A})-\mathrm{C}(21)-\mathrm{H}(21 \mathrm{~B}) & 108.1 \\ \mathrm{C}(21)-\mathrm{C}(22)-\mathrm{C}(23) & 110.30(14) \\ \mathrm{C}(21)-\mathrm{C}(22)-\mathrm{H}(22 \mathrm{~A}) & 109.6 \\ \mathrm{C}(23)-\mathrm{C}(22)-\mathrm{H}(22 \mathrm{~A}) & 109.6 \\ \mathrm{C}(21)-\mathrm{C}(22)-\mathrm{H}(22 \mathrm{~B}) & 109.6 \\ \mathrm{C}(23)-\mathrm{C}(22)-\mathrm{H}(22 \mathrm{~B}) & 109.6 \\ \mathrm{H}(22 \mathrm{~A})-\mathrm{C}(22)-\mathrm{H}(22 \mathrm{~B}) & 108.1 \\ \mathrm{C}(24)-\mathrm{C}(23)-\mathrm{C}(22) & 112.08(14) \\ \mathrm{C}(24)-\mathrm{C}(23)-\mathrm{H}(23 \mathrm{~A}) & 109.2 \\ \mathrm{C}(22)-\mathrm{C}(23)-\mathrm{H}(23 \mathrm{~A}) & 109.2 \\ \mathrm{C}(24)-\mathrm{C}(23)-\mathrm{H}(23 \mathrm{~B}) & 109.2 \\ \mathrm{C}(22)-\mathrm{C}(23)-\mathrm{H}(23 \mathrm{~B}) & 109.2 \\ \mathrm{H}(23 \mathrm{~A})-\mathrm{C}(23)-\mathrm{H}(23 \mathrm{~B}) & 107.9 \\ \mathrm{C}(19)-\mathrm{C}(24)-\mathrm{C}(23) & 120.75(14) \\ \mathrm{C}(19)-\mathrm{C}(24)-\mathrm{C}(14) & 120.49(15) \\ \mathrm{C}(23)-\mathrm{C}(24)-\mathrm{C}(14) & 118.40(13) \\ \end{array}$

Symmetry transformations used to generate equivalent atoms: 
Table S15. Anisotropic displacement parameters ( $\left.A^{\wedge} 2 \times 10^{\wedge} 3\right)$.

The anisotropic displacement factor exponent takes the form:

$-2 \mathrm{pi}^{\wedge} 2\left[\mathrm{~h}^{\wedge} 2 \mathrm{a}^{* \wedge} \mathrm{U} \mathrm{U} 11+\ldots+2 \mathrm{hk} \mathrm{a*} \mathrm{b}^{*} \mathrm{U} 12\right]$

\begin{tabular}{lcccccc}
\hline & & & & \\
$\mathrm{U} 11$ & $\mathrm{U} 22$ & $\mathrm{U} 33$ & $\mathrm{U} 23$ & $\mathrm{U} 13$ & $\mathrm{U} 12$ & \\
& & & & & & \\
$\mathrm{Br}(1)$ & $22(1)$ & $31(1)$ & $23(1)$ & $-5(1)$ & $7(1)$ & $-9(1)$ \\
$\mathrm{O}(1)$ & $25(1)$ & $21(1)$ & $36(1)$ & $4(1)$ & $6(1)$ & $4(1)$ \\
$\mathrm{O}(2)$ & $17(1)$ & $18(1)$ & $21(1)$ & $1(1)$ & $4(1)$ & $-1(1)$ \\
$\mathrm{O}(3)$ & $22(1)$ & $26(1)$ & $18(1)$ & $3(1)$ & $10(1)$ & $1(1)$ \\
$\mathrm{O}(4)$ & $32(1)$ & $31(1)$ & $18(1)$ & $3(1)$ & $9(1)$ & $4(1)$ \\
$\mathrm{C}(1)$ & $19(1)$ & $26(1)$ & $21(1)$ & $-5(1)$ & $11(1)$ & $-5(1)$ \\
$\mathrm{C}(2)$ & $26(1)$ & $20(1)$ & $30(1)$ & $-6(1)$ & $15(1)$ & $-5(1)$ \\
$\mathrm{C}(3)$ & $24(1)$ & $20(1)$ & $28(1)$ & $0(1)$ & $13(1)$ & $1(1)$ \\
$\mathrm{C}(4)$ & $19(1)$ & $21(1)$ & $22(1)$ & $-3(1)$ & $13(1)$ & $-1(1)$ \\
$\mathrm{C}(5)$ & $20(1)$ & $20(1)$ & $24(1)$ & $-1(1)$ & $9(1)$ & $-3(1)$ \\
$\mathrm{C}(6)$ & $22(1)$ & $22(1)$ & $25(1)$ & $1(1)$ & $9(1)$ & $-1(1)$ \\
$\mathrm{C}(7)$ & $20(1)$ & $19(1)$ & $23(1)$ & $1(1)$ & $12(1)$ & $1(1)$ \\
$\mathrm{C}(8)$ & $14(1)$ & $22(1)$ & $20(1)$ & $2(1)$ & $4(1)$ & $0(1)$ \\
$\mathrm{C}(9)$ & $14(1)$ & $21(1)$ & $16(1)$ & $0(1)$ & $6(1)$ & $0(1)$ \\
$\mathrm{C}(10)$ & $15(1)$ & $24(1)$ & $17(1)$ & $-1(1)$ & $5(1)$ & $0(1)$ \\
$\mathrm{C}(11)$ & $19(1)$ & $24(1)$ & $19(1)$ & $-4(1)$ & $6(1)$ & $-2(1)$ \\
$\mathrm{C}(12)$ & $17(1)$ & $20(1)$ & $25(1)$ & $-1(1)$ & $9(1)$ & $-2(1)$ \\
$\mathrm{C}(13)$ & $15(1)$ & $20(1)$ & $22(1)$ & $2(1)$ & $8(1)$ & $-1(1)$ \\
$\mathrm{C}(14)$ & $14(1)$ & $19(1)$ & $17(1)$ & $0(1)$ & $6(1)$ & $0(1)$ \\
$\mathrm{C}(15)$ & $18(1)$ & $29(1)$ & $19(1)$ & $1(1)$ & $4(1)$ & $2(1)$ \\
$\mathrm{C}(16)$ & $14(1)$ & $31(1)$ & $21(1)$ & $-2(1)$ & $5(1)$ & $0(1)$ \\
$\mathrm{C}(17)$ & $17(1)$ & $24(1)$ & $18(1)$ & $2(1)$ & $7(1)$ & $2(1)$ \\
$\mathrm{C}(18)$ & $24(1)$ & $17(1)$ & $21(1)$ & $-1(1)$ & $8(1)$ & $1(1)$ \\
$\mathrm{C}(19)$ & $19(1)$ & $15(1)$ & $20(1)$ & $-1(1)$ & $6(1)$ & $0(1)$ \\
$\mathrm{C}(20)$ & $19(1)$ & $23(1)$ & $22(1)$ & $-1(1)$ & $3(1)$ & $2(1)$ \\
$\mathrm{C}(21)$ & $16(1)$ & $26(1)$ & $26(1)$ & $-2(1)$ & $3(1)$ & $3(1)$ \\
$\mathrm{C}(22)$ & $18(1)$ & $25(1)$ & $25(1)$ & $-1(1)$ & $7(1)$ & $3(1)$ \\
$\mathrm{C}(23)$ & $16(1)$ & $20(1)$ & $20(1)$ & $0(1)$ & $7(1)$ & $2(1)$ \\
$\mathrm{C}(24)$ & $16(1)$ & $14(1)$ & $20(1)$ & $0(1)$ & $6(1)$ & $-1(1)$ \\
& & & & & & \\
\hline & & & & & & \\
\end{tabular}


Table S16. Hydrogen coordinates ( x 10^4) and isotropic displacement parameters $\left(\mathrm{A}^{\wedge} 2 \times 10^{\wedge} 3\right)$.

\begin{tabular}{|c|c|c|c|c|}
\hline \multirow{2}{*}{$\begin{array}{l}\mathrm{x} \\
\mathrm{H}(2)\end{array}$} & \multirow{2}{*}{$\begin{array}{l}\mathrm{y} \\
\\
\end{array} 6672$} & $\begin{array}{l}\mathrm{Z} \\
\\
85\end{array}$ & $\mathrm{U}(\mathrm{eq})$ & \\
\hline & & 8539 & 9231 & 29 \\
\hline $\mathrm{H}(3)$ & 4585 & 8378 & 7039 & 27 \\
\hline $\mathrm{H}(5)$ & 5073 & 6809 & 8035 & 26 \\
\hline $\mathrm{H}(6)$ & 7164 & 6966 & 10231 & 27 \\
\hline $\mathrm{H}(8 \mathrm{~A})$ & 1596 & 7032 & 3168 & 24 \\
\hline $\mathrm{H}(8 \mathrm{~B})$ & 1030 & 6933 & 4752 & 24 \\
\hline $\mathrm{H}(9)$ & 2420 & 6181 & 3429 & 20 \\
\hline $\mathrm{H}(11 \mathrm{~A})$ & -316 & 5362 & 1164 & 25 \\
\hline $\mathrm{H}(11 \mathrm{~B})$ & 1178 & 5484 & 1329 & 25 \\
\hline $\mathrm{H}(12)$ & 899 & 4779 & 3437 & 25 \\
\hline $\mathrm{H}(13)$ & 1924 & 5081 & 6211 & 23 \\
\hline $\mathrm{H}(15 \mathrm{~A})$ & -595 & 6246 & -226 & 34 \\
\hline $\mathrm{H}(15 \mathrm{~B})$ & 924 & 6431 & 492 & 34 \\
\hline $\mathrm{H}(15 \mathrm{C})$ & -155 & 6765 & 991 & 34 \\
\hline $\mathrm{H}(16 \mathrm{~A})$ & -868 & 6471 & 3512 & 33 \\
\hline $\mathrm{H}(16 \mathrm{~B})$ & -735 & 5857 & 4014 & 33 \\
\hline $\mathrm{H}(16 \mathrm{C})$ & -1633 & 6042 & 2028 & 33 \\
\hline $\mathrm{H}(17 \mathrm{~A})$ & 1877 & 6491 & 7350 & 23 \\
\hline $\mathrm{H}(17 \mathrm{~B})$ & 595 & 6122 & 6546 & 23 \\
\hline $\mathrm{H}(20 \mathrm{~A})$ & 5788 & 5390 & 10086 & 27 \\
\hline $\mathrm{H}(20 \mathrm{~B})$ & 5945 & 6012 & 10449 & 27 \\
\hline $\mathrm{H}(21 \mathrm{~A})$ & 6691 & 6154 & 8169 & 29 \\
\hline $\mathrm{H}(21 \mathrm{~B})$ & 7355 & 5596 & 8926 & 29 \\
\hline $\mathrm{H}(22 \mathrm{~A})$ & 5605 & 5155 & 6645 & 27 \\
\hline $\mathrm{H}(22 \mathrm{~B})$ & 6364 & 5549 & 5766 & 27 \\
\hline $\mathrm{H}(23 \mathrm{~A})$ & 4685 & 6177 & 5187 & 22 \\
\hline $\mathrm{H}(23 \mathrm{~B})$ & 3990 & 5627 & 4394 & 22 \\
\hline
\end{tabular}


Table S17. Torsion angles [deg].

\begin{tabular}{|c|c|}
\hline $\mathrm{C}(6)-\mathrm{C}(1)-\mathrm{C}(2)-\mathrm{C}(3)$ & $-0.4(3)$ \\
\hline $\mathrm{Br}(1)-\mathrm{C}(1)-\mathrm{C}(2)-\mathrm{C}(3)$ & $-179.44(13)$ \\
\hline $\mathrm{C}(1)-\mathrm{C}(2)-\mathrm{C}(3)-\mathrm{C}(4)$ & $-0.1(3)$ \\
\hline $\mathrm{C}(2)-\mathrm{C}(3)-\mathrm{C}(4)-\mathrm{C}(5)$ & $0.6(3)$ \\
\hline $\mathrm{C}(2)-\mathrm{C}(3)-\mathrm{C}(4)-\mathrm{C}(7)$ & $-178.83(16)$ \\
\hline$C(3)-C(4)-C(5)-C(6)$ & $-0.6(3)$ \\
\hline$C(7)-C(4)-C(5)-C(6)$ & $178.81(16)$ \\
\hline $\mathrm{C}(4)-\mathrm{C}(5)-\mathrm{C}(6)-\mathrm{C}(1)$ & $0.1(3)$ \\
\hline $\mathrm{C}(2)-\mathrm{C}(1)-\mathrm{C}(6)-\mathrm{C}(5)$ & $0.4(3)$ \\
\hline $\mathrm{Br}(1)-\mathrm{C}(1)-\mathrm{C}(6)-\mathrm{C}(5)$ & $179.44(14)$ \\
\hline $\mathrm{C}(8)-\mathrm{O}(2)-\mathrm{C}(7)-\mathrm{O}(1)$ & $-2.5(2)$ \\
\hline $\mathrm{C}(8)-\mathrm{O}(2)-\mathrm{C}(7)-\mathrm{C}(4)$ & $177.97(13)$ \\
\hline $\mathrm{C}(5)-\mathrm{C}(4)-\mathrm{C}(7)-\mathrm{O}(1)$ & $177.30(18)$ \\
\hline $\mathrm{C}(3)-\mathrm{C}(4)-\mathrm{C}(7)-\mathrm{O}(1)$ & $-3.3(3)$ \\
\hline $\mathrm{C}(5)-\mathrm{C}(4)-\mathrm{C}(7)-\mathrm{O}(2)$ & $-3.2(2)$ \\
\hline $\mathrm{C}(3)-\mathrm{C}(4)-\mathrm{C}(7)-\mathrm{O}(2)$ & $176.25(14)$ \\
\hline $\mathrm{C}(7)-\mathrm{O}(2)-\mathrm{C}(8)-\mathrm{C}(9)$ & 169.87(13) \\
\hline $\mathrm{O}(2)-\mathrm{C}(8)-\mathrm{C}(9)-\mathrm{C}(10)$ & $-177.33(12)$ \\
\hline $\mathrm{O}(2)-\mathrm{C}(8)-\mathrm{C}(9)-\mathrm{C}(14)$ & $52.00(17)$ \\
\hline $\mathrm{C}(8)-\mathrm{C}(9)-\mathrm{C}(10)-\mathrm{C}(15)$ & $52.95(18)$ \\
\hline $\mathrm{C}(14)-\mathrm{C}(9)-\mathrm{C}(10)-\mathrm{C}(15)$ & $-176.23(13)$ \\
\hline $\mathrm{C}(8)-\mathrm{C}(9)-\mathrm{C}(10)-\mathrm{C}(16)$ & $-68.99(18)$ \\
\hline $\mathrm{C}(14)-\mathrm{C}(9)-\mathrm{C}(10)-\mathrm{C}(16)$ & $61.83(19)$ \\
\hline $\mathrm{C}(8)-\mathrm{C}(9)-\mathrm{C}(10)-\mathrm{C}(11)$ & $169.94(14)$ \\
\hline$C(14)-C(9)-C(10)-C(11)$ & $-59.23(17)$ \\
\hline $\mathrm{C}(15)-\mathrm{C}(10)-\mathrm{C}(11)-\mathrm{C}(12)$ & $169.96(14)$ \\
\hline $\mathrm{C}(16)-\mathrm{C}(10)-\mathrm{C}(11)-\mathrm{C}(12)$ & $-72.67(18)$ \\
\hline $\mathrm{C}(9)-\mathrm{C}(10)-\mathrm{C}(11)-\mathrm{C}(12)$ & $51.29(18)$ \\
\hline $\mathrm{C}(10)-\mathrm{C}(11)-\mathrm{C}(12)-\mathrm{C}(13)$ & $-21.7(2)$ \\
\hline $\mathrm{C}(11)-\mathrm{C}(12)-\mathrm{C}(13)-\mathrm{C}(14)$ & $-5.2(3)$ \\
\hline $\mathrm{C}(12)-\mathrm{C}(13)-\mathrm{C}(14)-\mathrm{C}(17)$ & $123.47(18)$ \\
\hline $\mathrm{C}(12)-\mathrm{C}(13)-\mathrm{C}(14)-\mathrm{C}(24)$ & $-122.22(17)$ \\
\hline $\mathrm{C}(12)-\mathrm{C}(13)-\mathrm{C}(14)-\mathrm{C}(9)$ & $-1.2(2)$ \\
\hline $\mathrm{C}(8)-\mathrm{C}(9)-\mathrm{C}(14)-\mathrm{C}(13)$ & $163.49(13)$ \\
\hline$C(10)-C(9)-C(14)-C(13)$ & $34.28(18)$ \\
\hline $\mathrm{C}(8)-\mathrm{C}(9)-\mathrm{C}(14)-\mathrm{C}(17)$ & $39.01(18)$ \\
\hline $\mathrm{C}(10)-\mathrm{C}(9)-\mathrm{C}(14)-\mathrm{C}(17)$ & $-90.20(16)$ \\
\hline $\mathrm{C}(8)-\mathrm{C}(9)-\mathrm{C}(14)-\mathrm{C}(24)$ & $-80.98(17)$ \\
\hline $\mathrm{C}(10)-\mathrm{C}(9)-\mathrm{C}(14)-\mathrm{C}(24)$ & $149.80(14)$ \\
\hline $\mathrm{C}(18)-\mathrm{O}(3)-\mathrm{C}(17)-\mathrm{C}(14)$ & $55.51(18)$ \\
\hline $\mathrm{C}(13)-\mathrm{C}(14)-\mathrm{C}(17)-\mathrm{O}(3)$ & $58.41(17)$ \\
\hline $\mathrm{C}(24)-\mathrm{C}(14)-\mathrm{C}(17)-\mathrm{O}(3)$ & $-53.92(17)$ \\
\hline $\mathrm{C}(9)-\mathrm{C}(14)-\mathrm{C}(17)-\mathrm{O}(3)$ & $-177.30(13)$ \\
\hline $\mathrm{C}(17)-\mathrm{O}(3)-\mathrm{C}(18)-\mathrm{O}(4)$ & $160.90(15)$ \\
\hline $\mathrm{C}(17)-\mathrm{O}(3)-\mathrm{C}(18)-\mathrm{C}(19)$ & $-23.0(2)$ \\
\hline $\mathrm{O}(4)-\mathrm{C}(18)-\mathrm{C}(19)-\mathrm{C}(24)$ & $168.14(17)$ \\
\hline $\mathrm{O}(3)-\mathrm{C}(18)-\mathrm{C}(19)-\mathrm{C}(24)$ & $-7.8(2)$ \\
\hline $\mathrm{O}(4)-\mathrm{C}(18)-\mathrm{C}(19)-\mathrm{C}(20)$ & $-4.1(2)$ \\
\hline
\end{tabular}


$\mathrm{O}(3)-\mathrm{C}(18)-\mathrm{C}(19)-\mathrm{C}(20)$

$\mathrm{C}(24)-\mathrm{C}(19)-\mathrm{C}(20)-\mathrm{C}(21)$

$\mathrm{C}(18)-\mathrm{C}(19)-\mathrm{C}(20)-\mathrm{C}(21)$

$\mathrm{C}(19)-\mathrm{C}(20)-\mathrm{C}(21)-\mathrm{C}(22)$

$\mathrm{C}(20)-\mathrm{C}(21)-\mathrm{C}(22)-\mathrm{C}(23)$

$\mathrm{C}(21)-\mathrm{C}(22)-\mathrm{C}(23)-\mathrm{C}(24)$

C(18)-C(19)-C(24)-C(23)

$\mathrm{C}(20)-\mathrm{C}(19)-\mathrm{C}(24)-\mathrm{C}(23)$

$\mathrm{C}(18)-\mathrm{C}(19)-\mathrm{C}(24)-\mathrm{C}(14)$

C(20)-C(19)-C(24)-C(14)

$\mathrm{C}(22)-\mathrm{C}(23)-\mathrm{C}(24)-\mathrm{C}(19)$

$\mathrm{C}(22)-\mathrm{C}(23)-\mathrm{C}(24)-\mathrm{C}(14)$

$\mathrm{C}(13)-\mathrm{C}(14)-\mathrm{C}(24)-\mathrm{C}(19)$

$\mathrm{C}(17)-\mathrm{C}(14)-\mathrm{C}(24)-\mathrm{C}(19)$

$\mathrm{C}(9)-\mathrm{C}(14)-\mathrm{C}(24)-\mathrm{C}(19)$

$\mathrm{C}(13)-\mathrm{C}(14)-\mathrm{C}(24)-\mathrm{C}(23)$

$\mathrm{C}(17)-\mathrm{C}(14)-\mathrm{C}(24)-\mathrm{C}(23)$

$\mathrm{C}(9)-\mathrm{C}(14)-\mathrm{C}(24)-\mathrm{C}(23)$
179.95(14)

5.7(2)

177.64(14)

$-37.1(2)$

$60.97(19)$

$-52.36(19)$

$-168.61(15)$

2.9(3)

$4.5(2)$

$175.95(15)$

$20.8(2)$

$-152.48(14)$

$-91.84(18)$

$25.6(2)$

148.35(15)

81.41(17)

$-161.14(14)$

$-38.40(19)$ 


\section{IV. ${ }^{1} \mathrm{H}$ and ${ }^{13} \mathrm{C}$ NMR for Synthetic Compounds}

1H NMR (CDCl3, $400 \mathrm{MHz})$ :

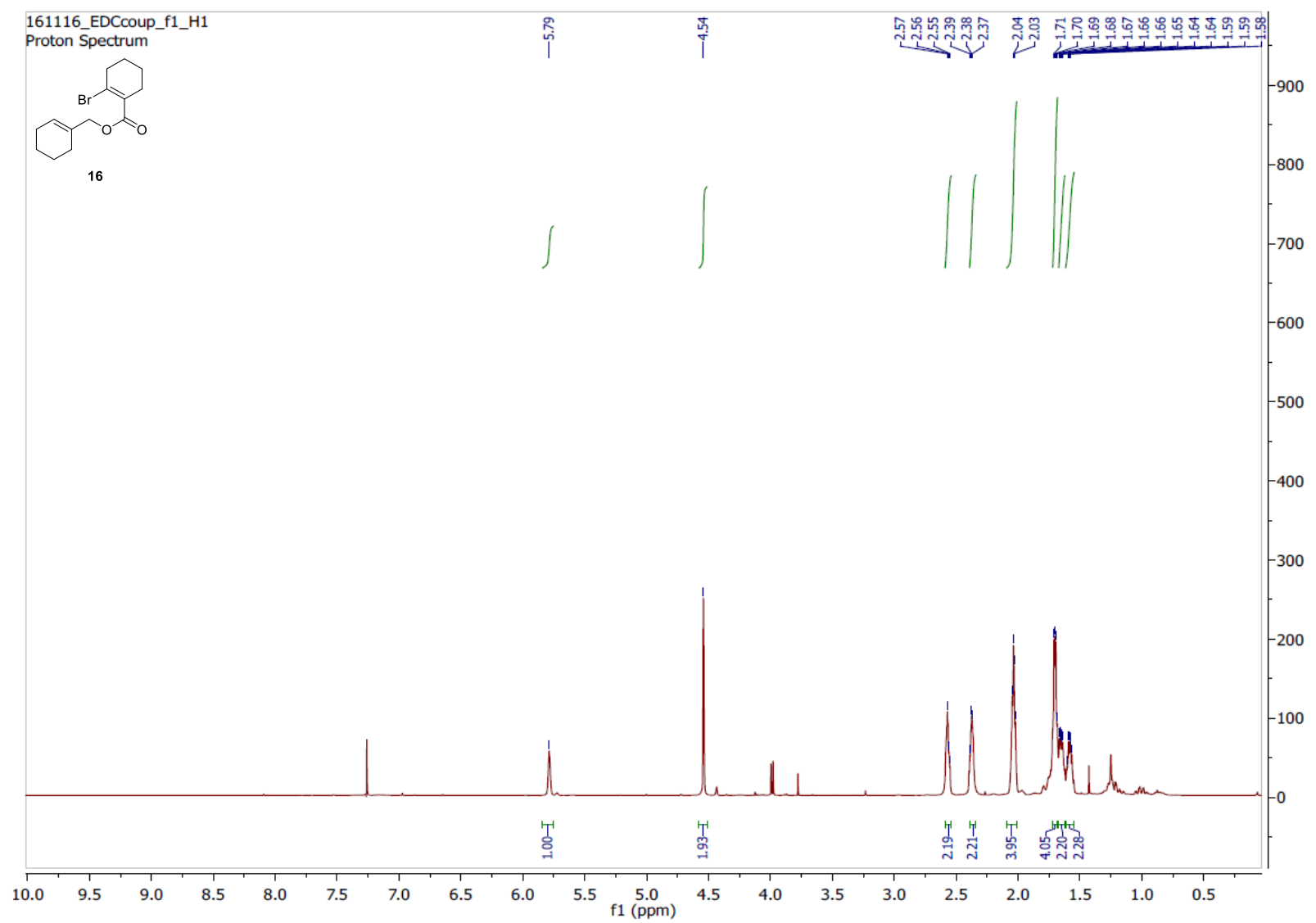


13C $\{1$ H $\}$ NMR (CDCl3, $100 \mathrm{MHz})$ :

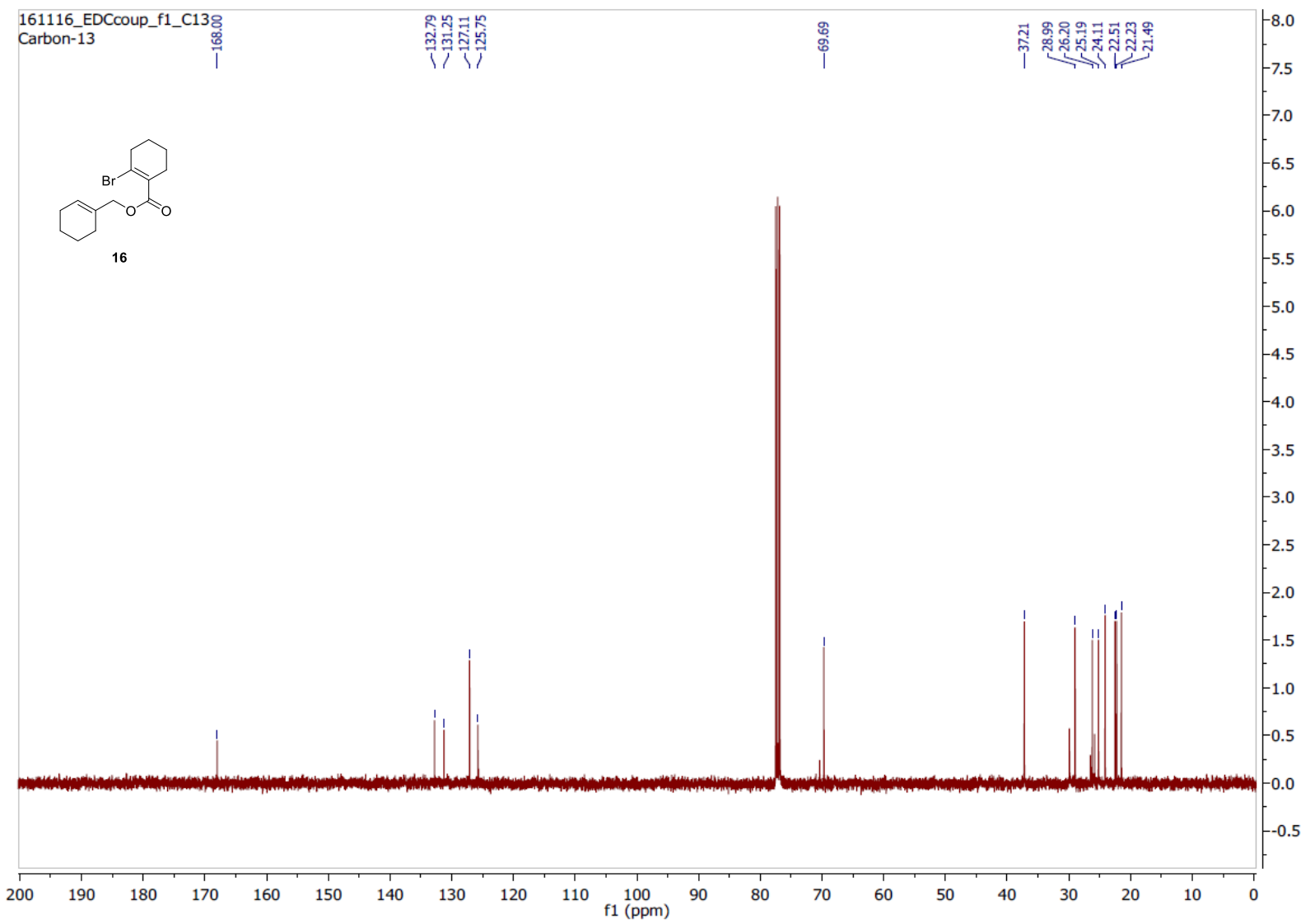


1H NMR (CDCl3, $500 \mathrm{MHz})$ :

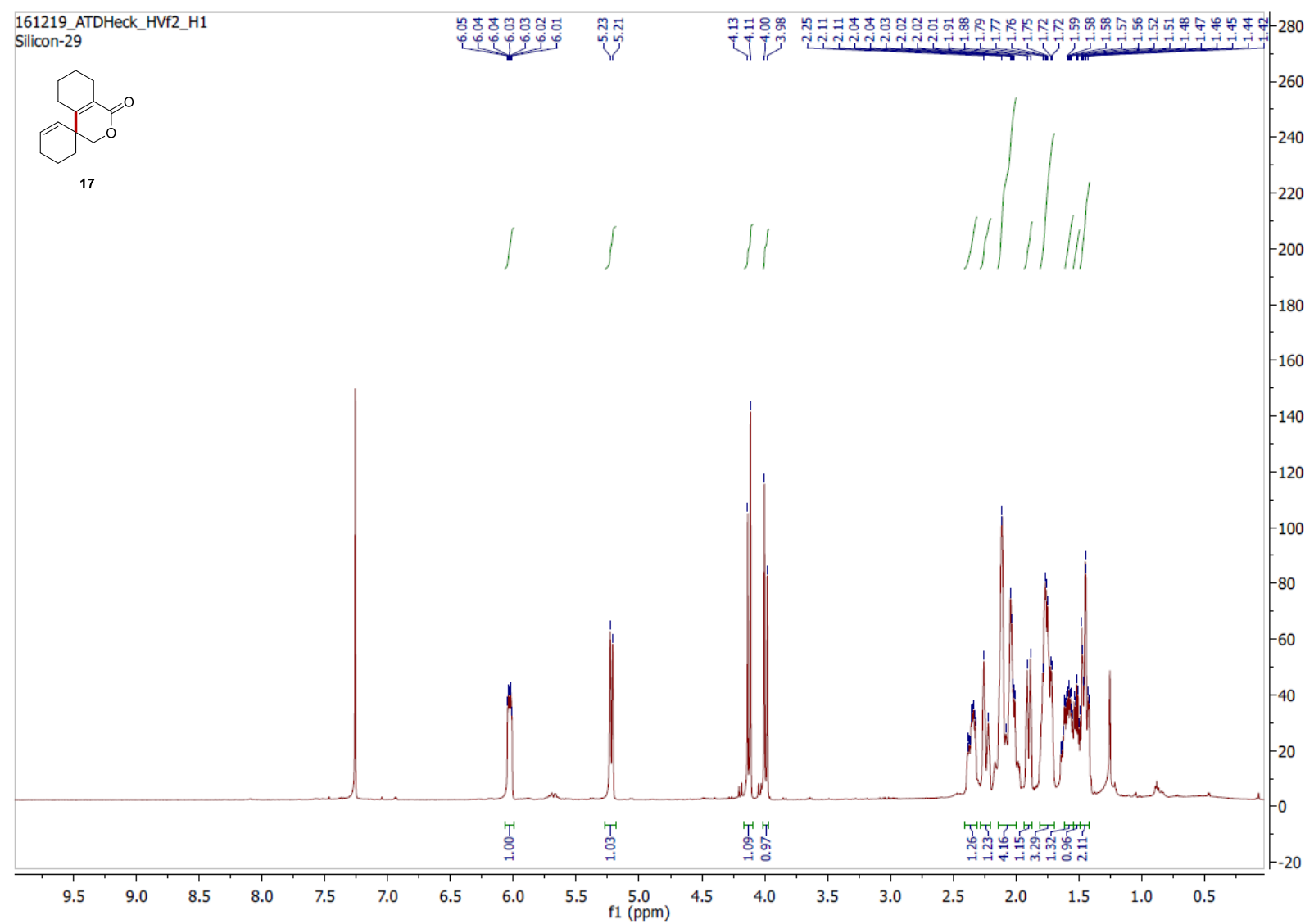


13C $\{1$ H $\}$ NMR (CDCl3, $126 \mathrm{MHz})$ :

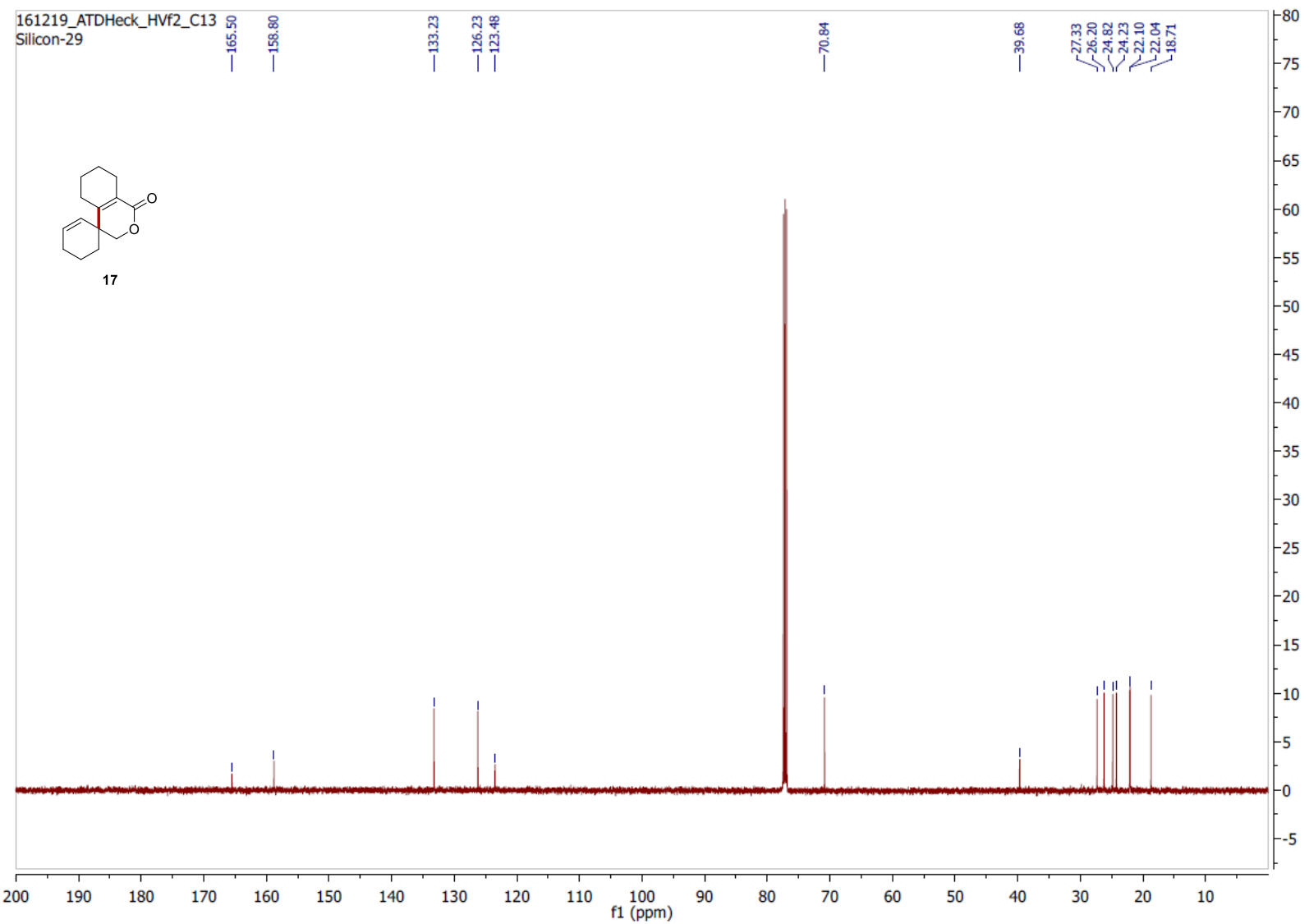


1H NMR (CDCl3, $400 \mathrm{MHz}$ ):

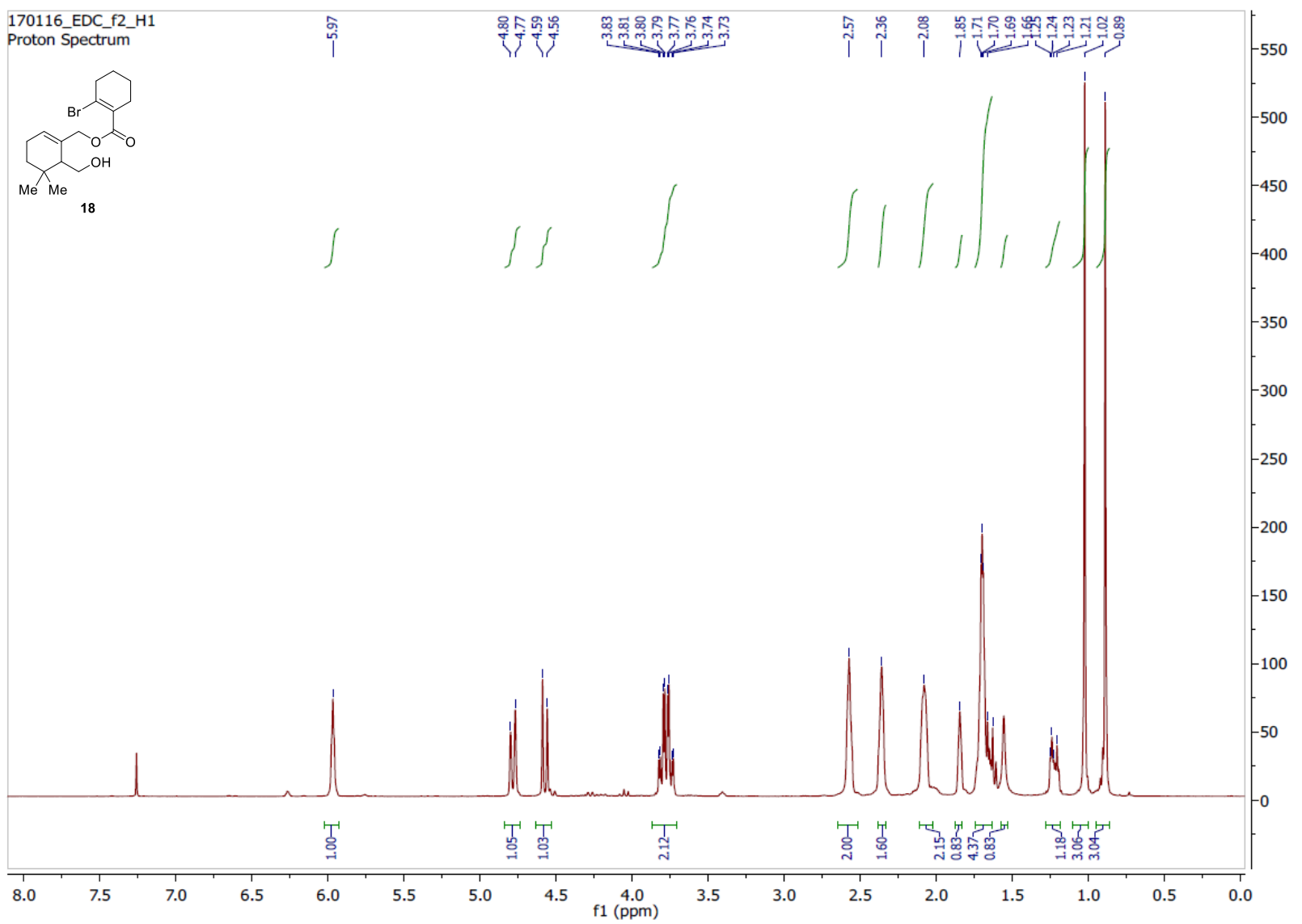


13C $\{1$ H $\}$ NMR (CDCl3, $100 \mathrm{MHz})$ :

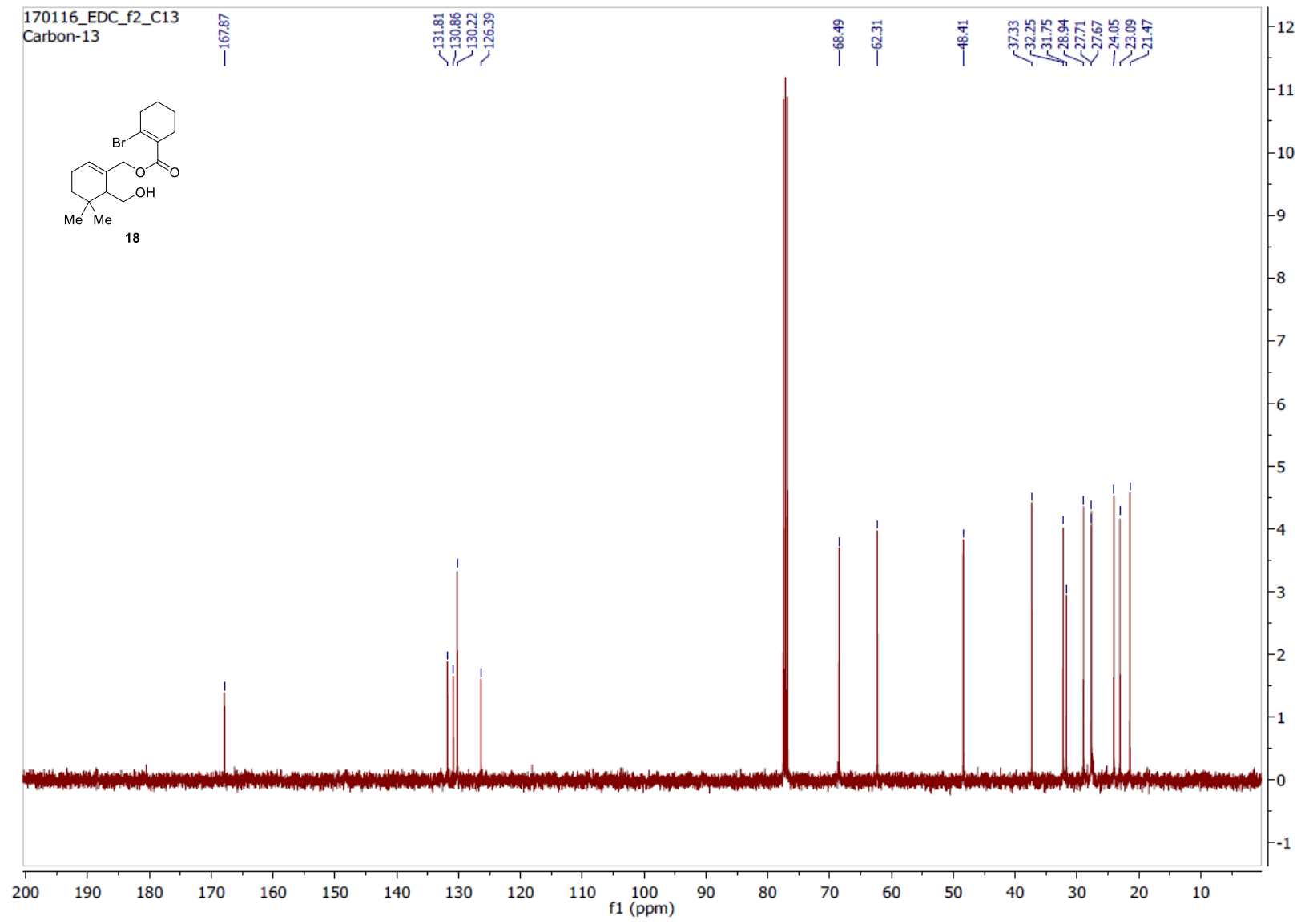


1H NMR (CDCl3, $400 \mathrm{MHz})$ :

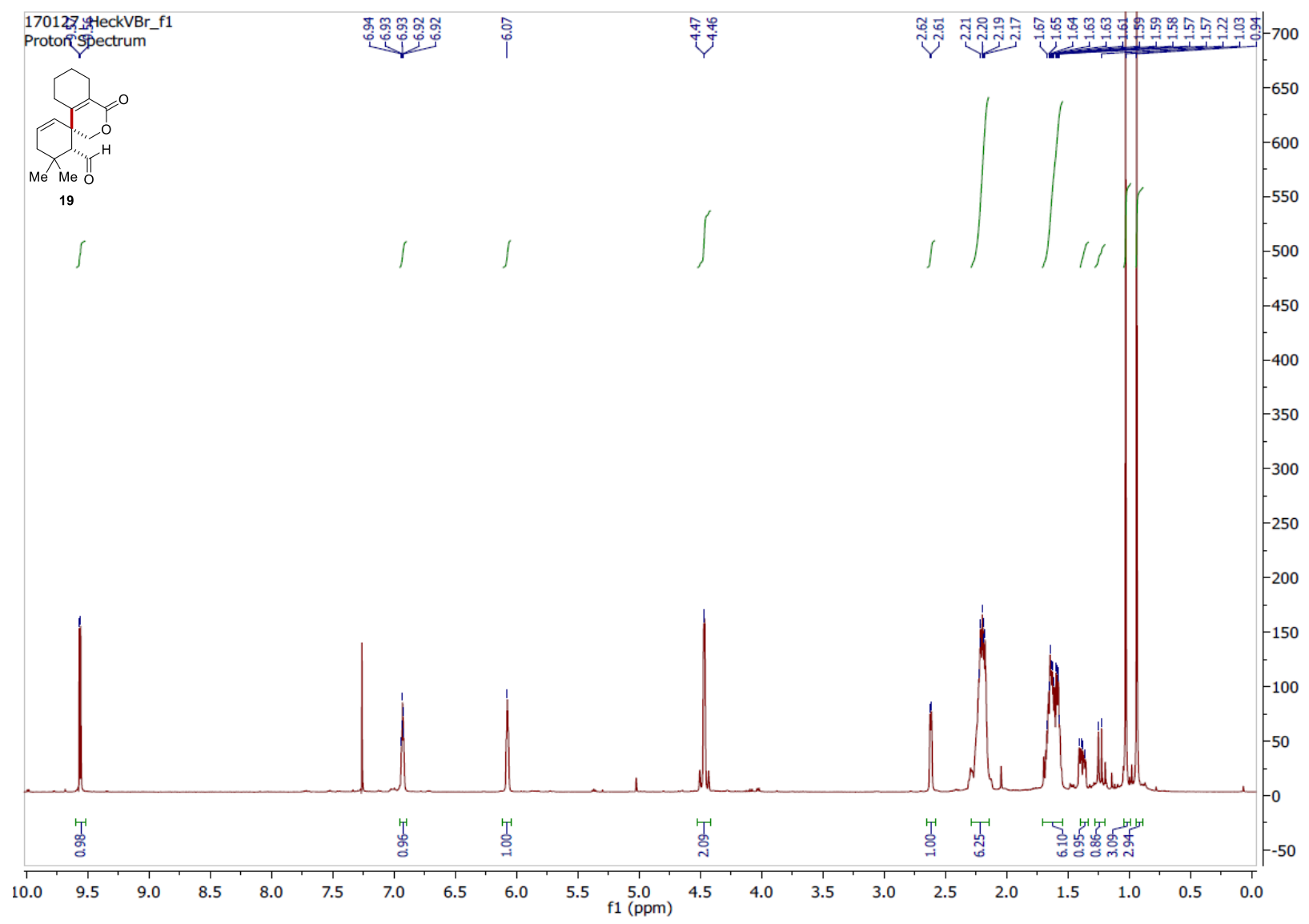


13C $\{1$ H $\}$ NMR (CDCl3, $100 \mathrm{MHz})$ :

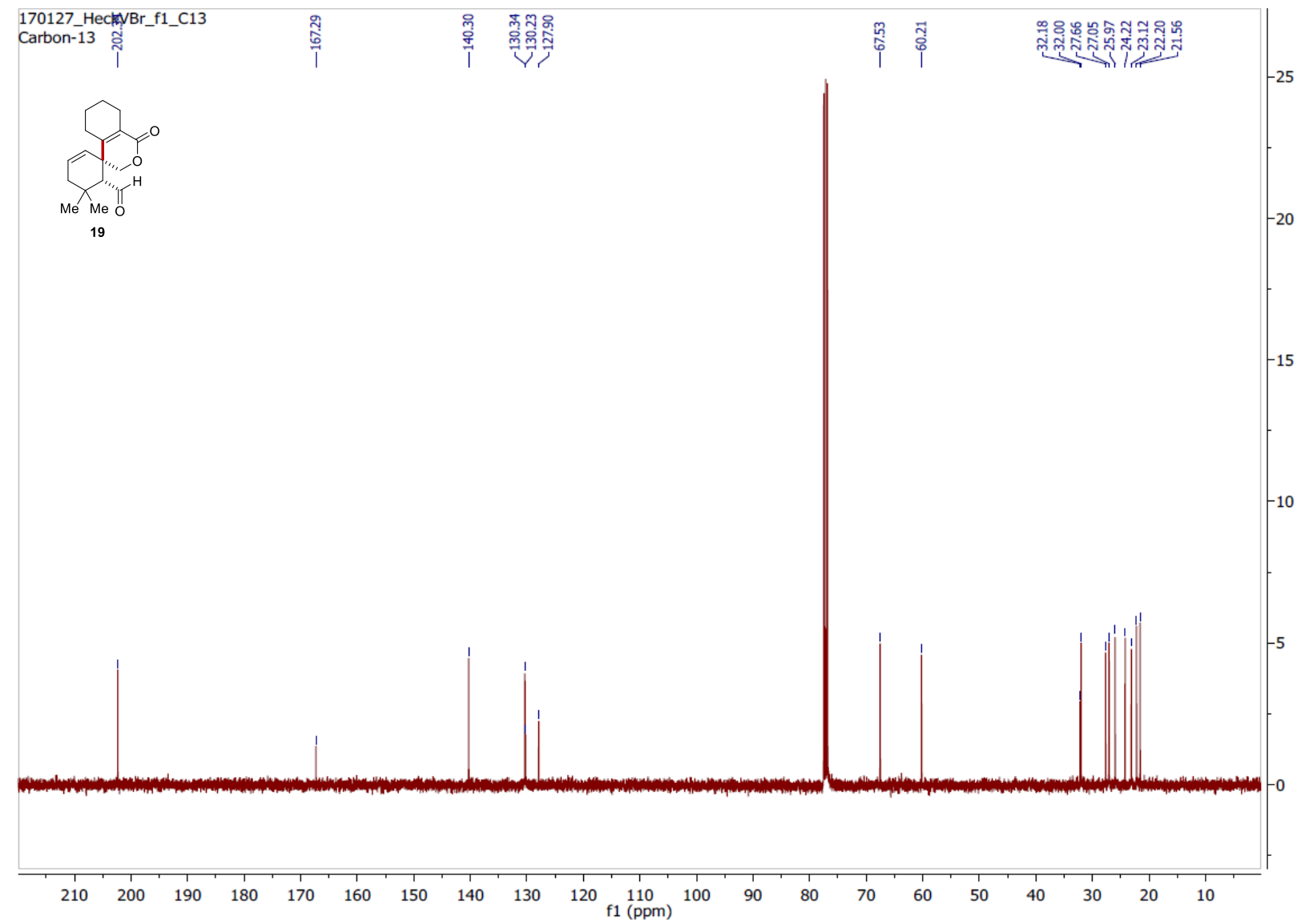


1H NMR (CDCl3, $500 \mathrm{MHz}$ ):

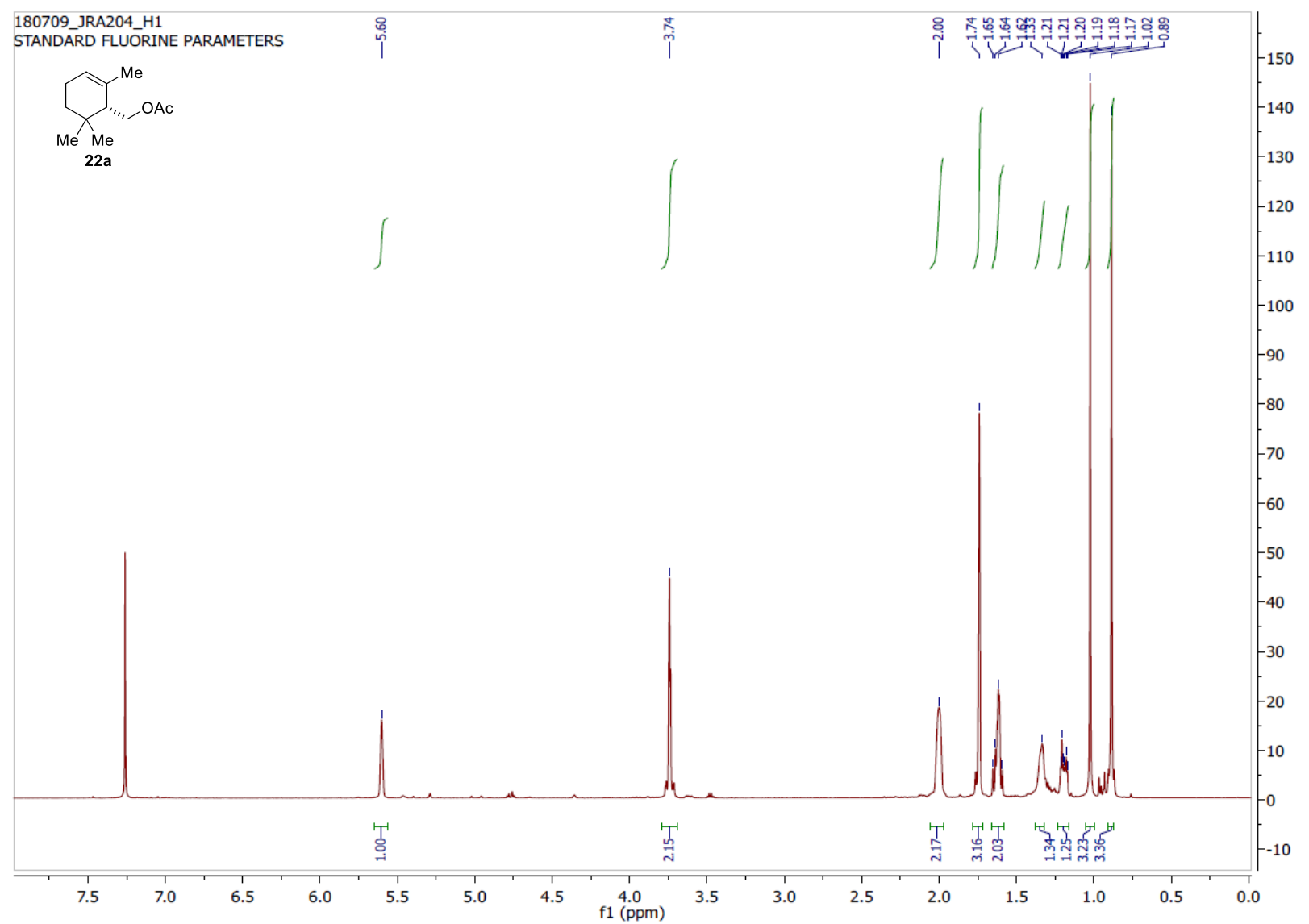


1H NMR (CDCl3, $700 \mathrm{MHz})$ :

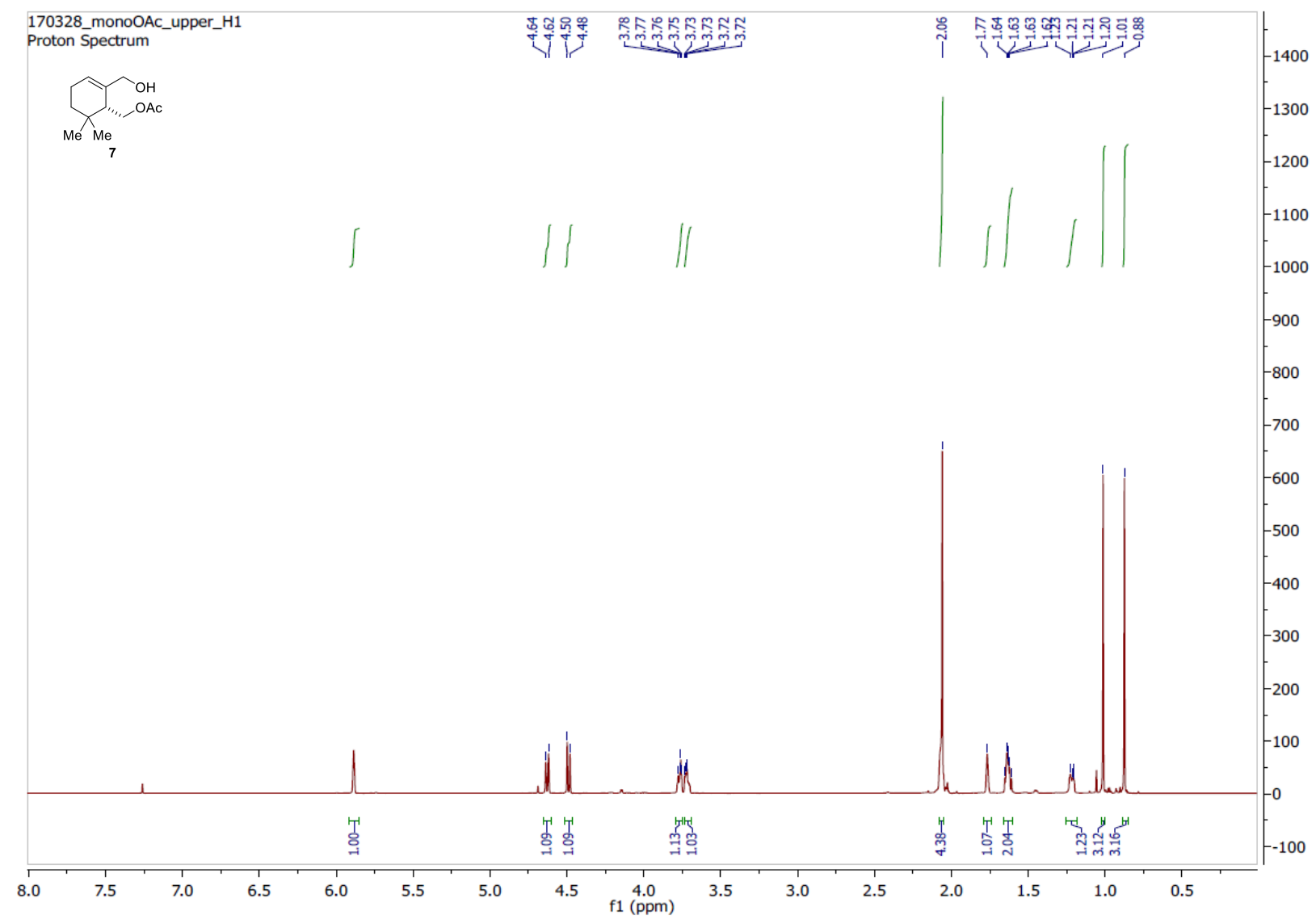


13C $\{1$ H $\}$ NMR $(\mathrm{CDCl} 3,176 \mathrm{MHz})$ :

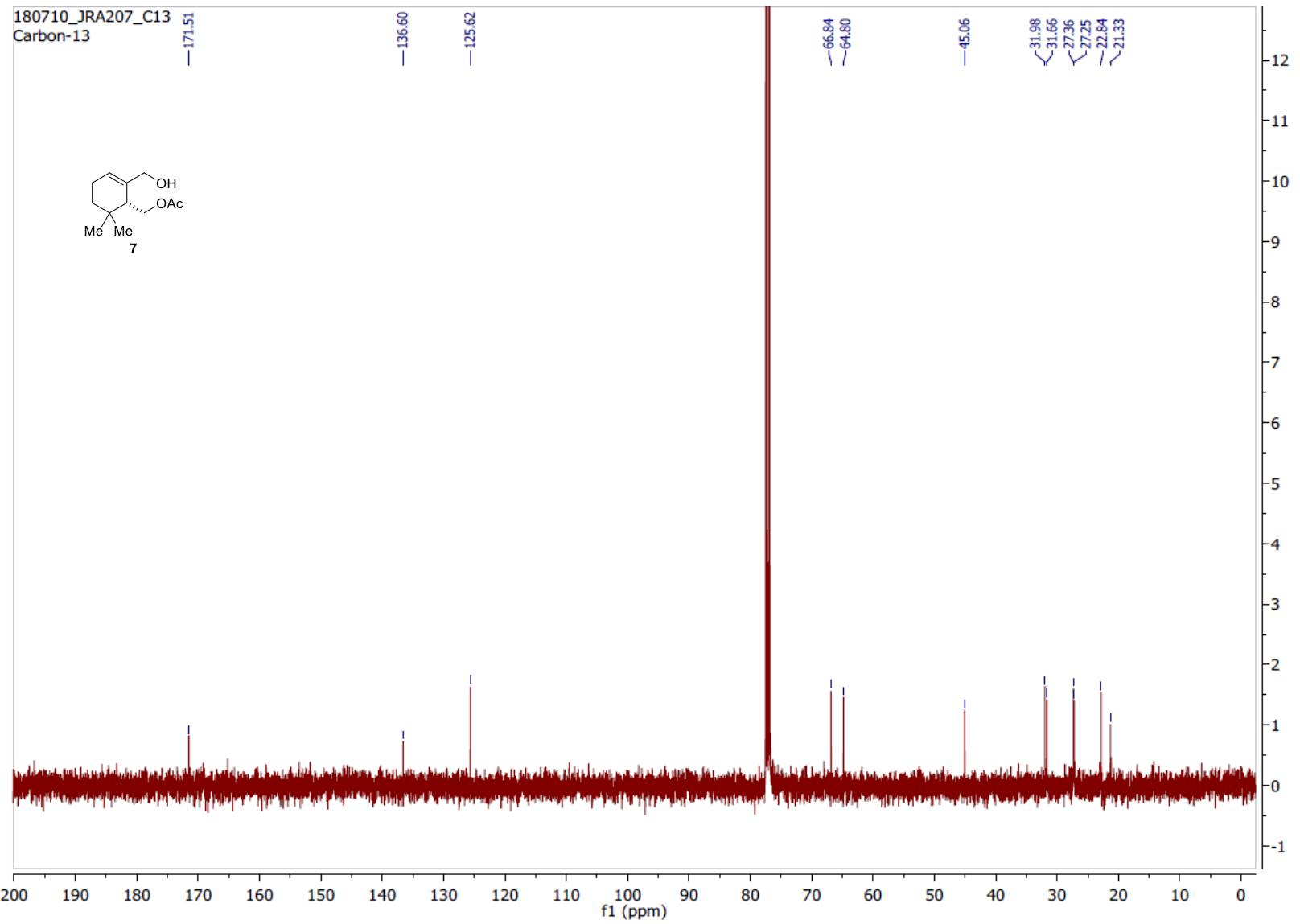


1H NMR (CDCl3, $700 \mathrm{MHz}$ ):

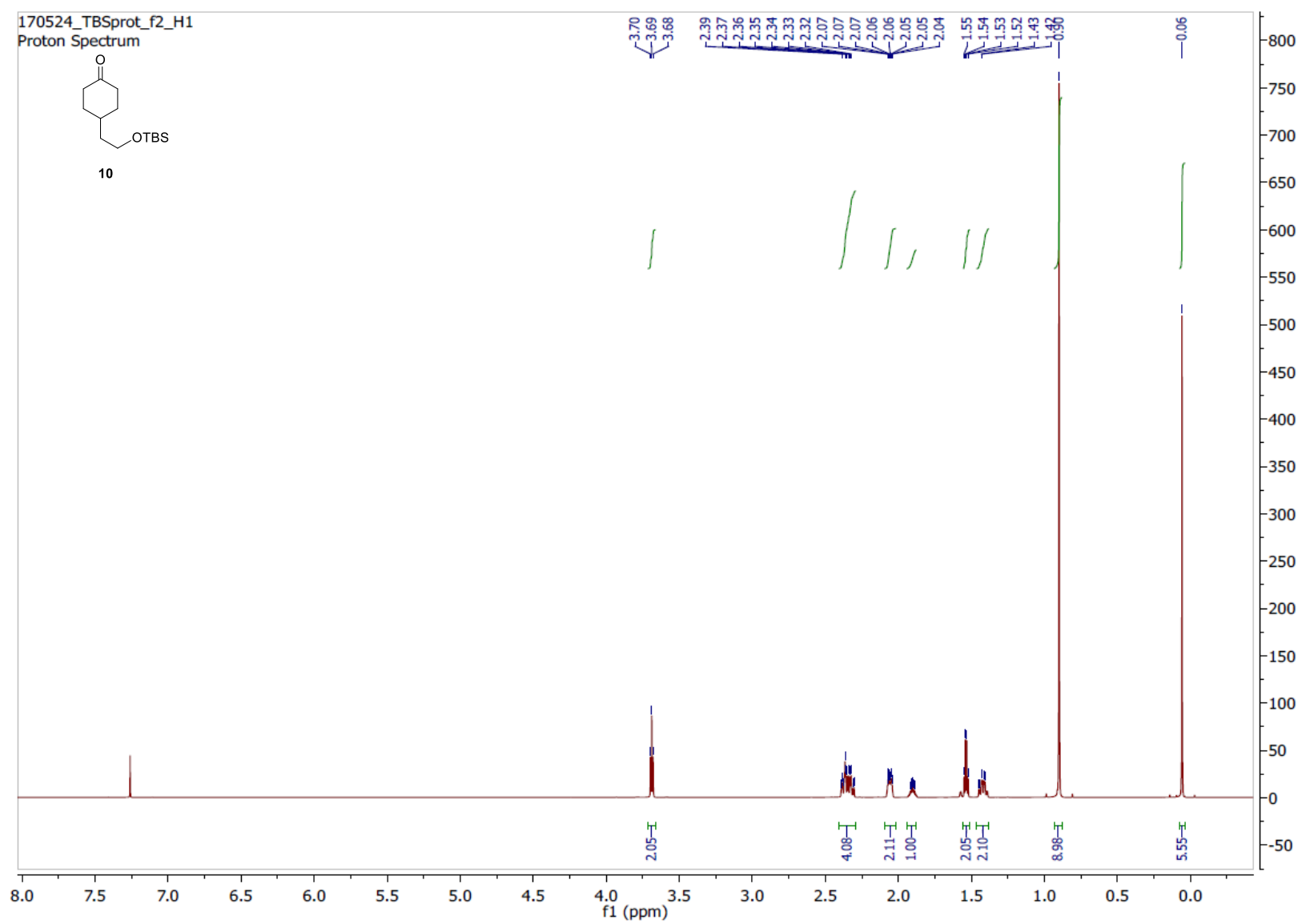


13C $\{1$ H $\}$ NMR (CDCl3, $176 \mathrm{MHz})$ :

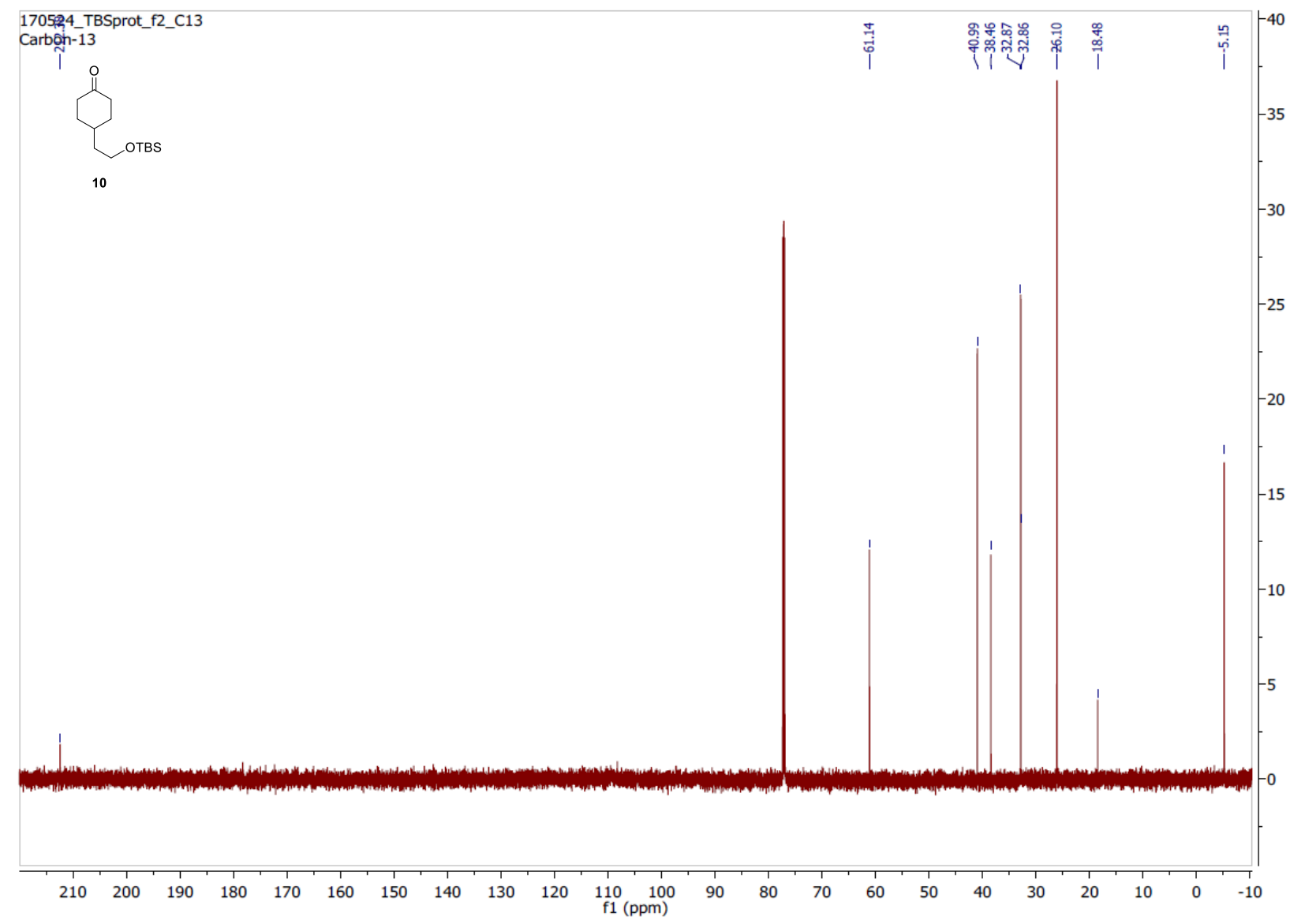


1H NMR (CD2Cl2, $400 \mathrm{MHz}$ ):

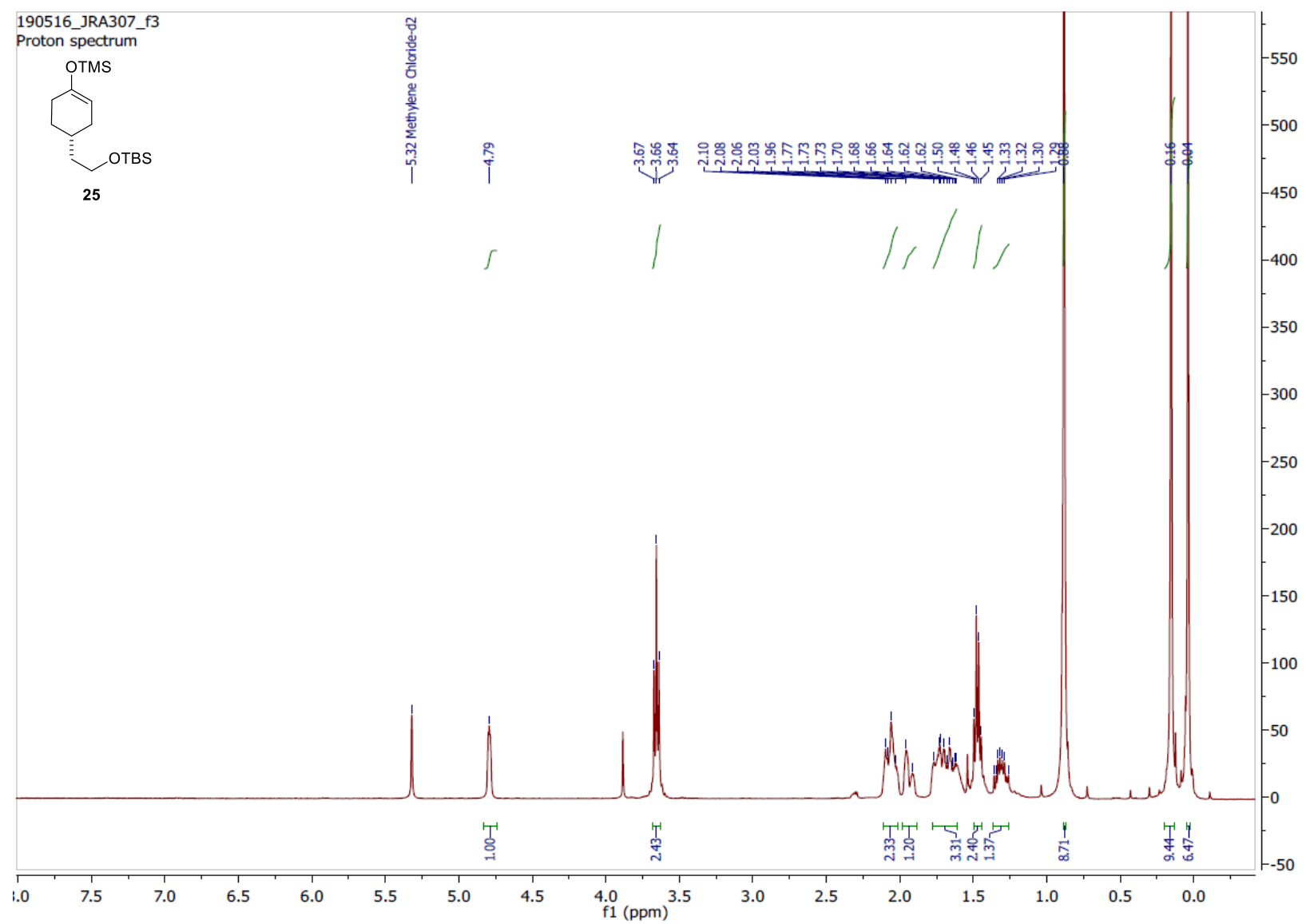


13C $\{1$ H $\}$ NMR (CD2Cl2, $176 \mathrm{MHz})$ :

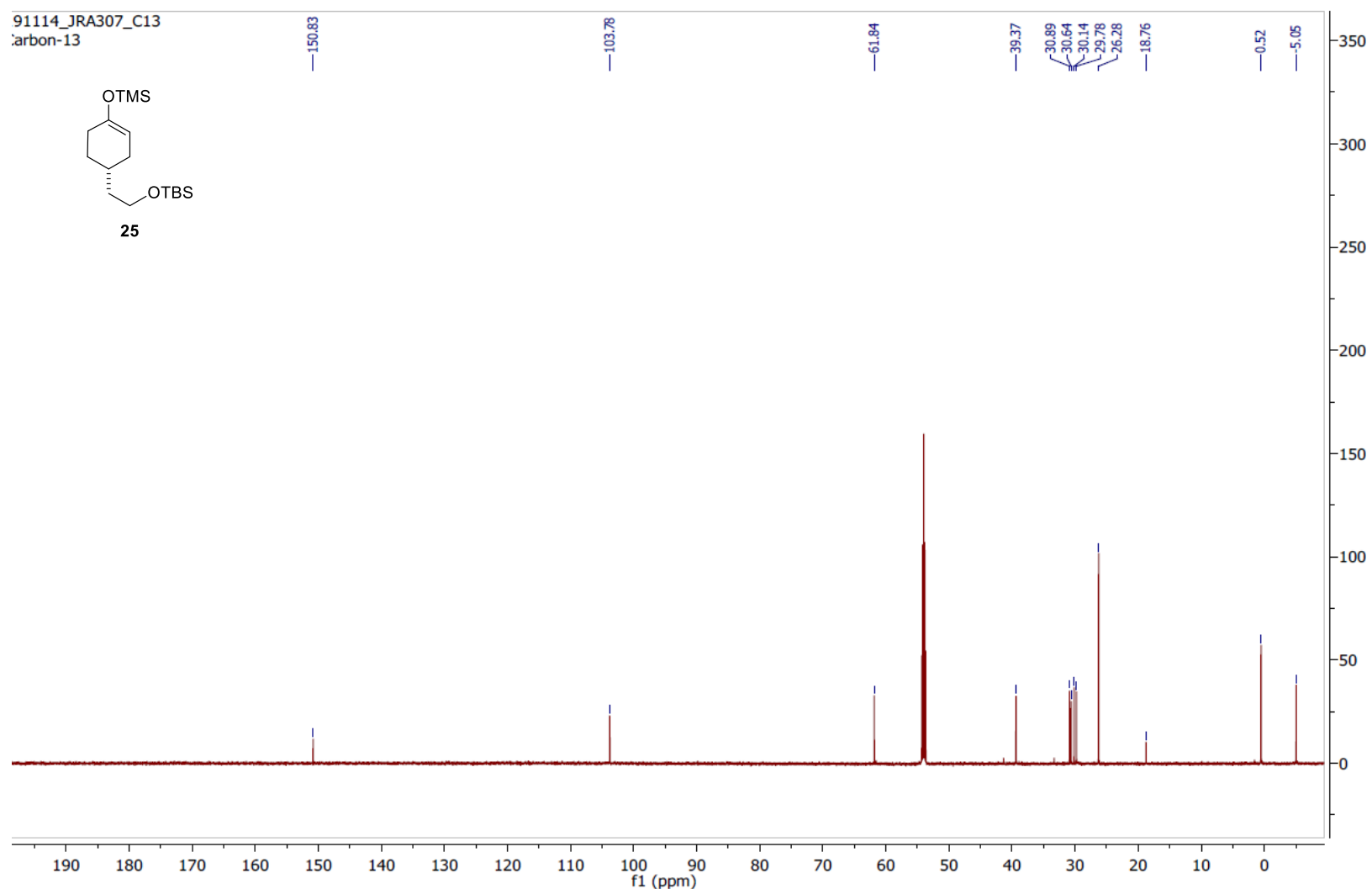


1H NMR (CDCl3, $400 \mathrm{MHz})$ :

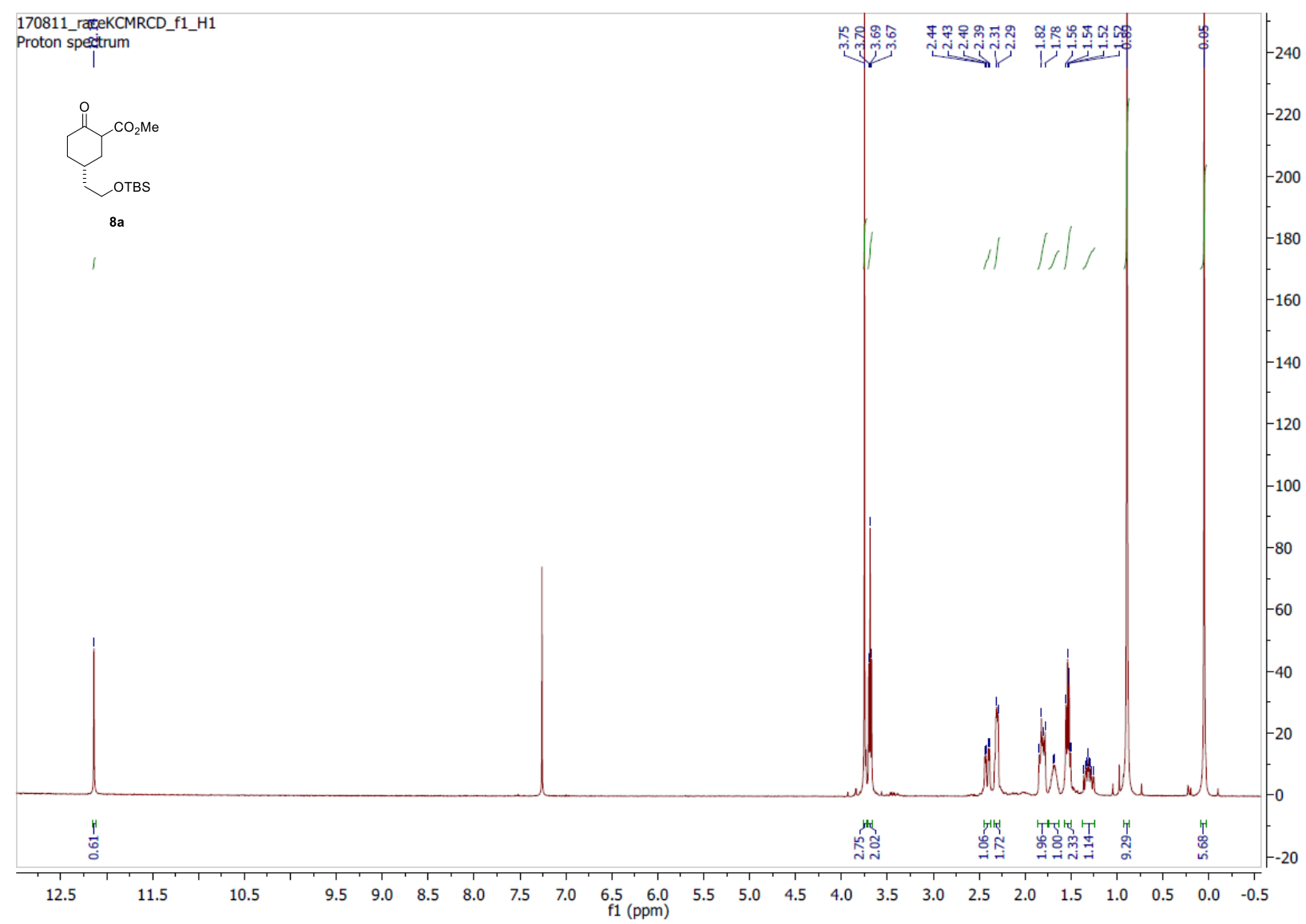


13C $\{1$ H $\}$ NMR (CDCl3, $101 \mathrm{MHz})$ :

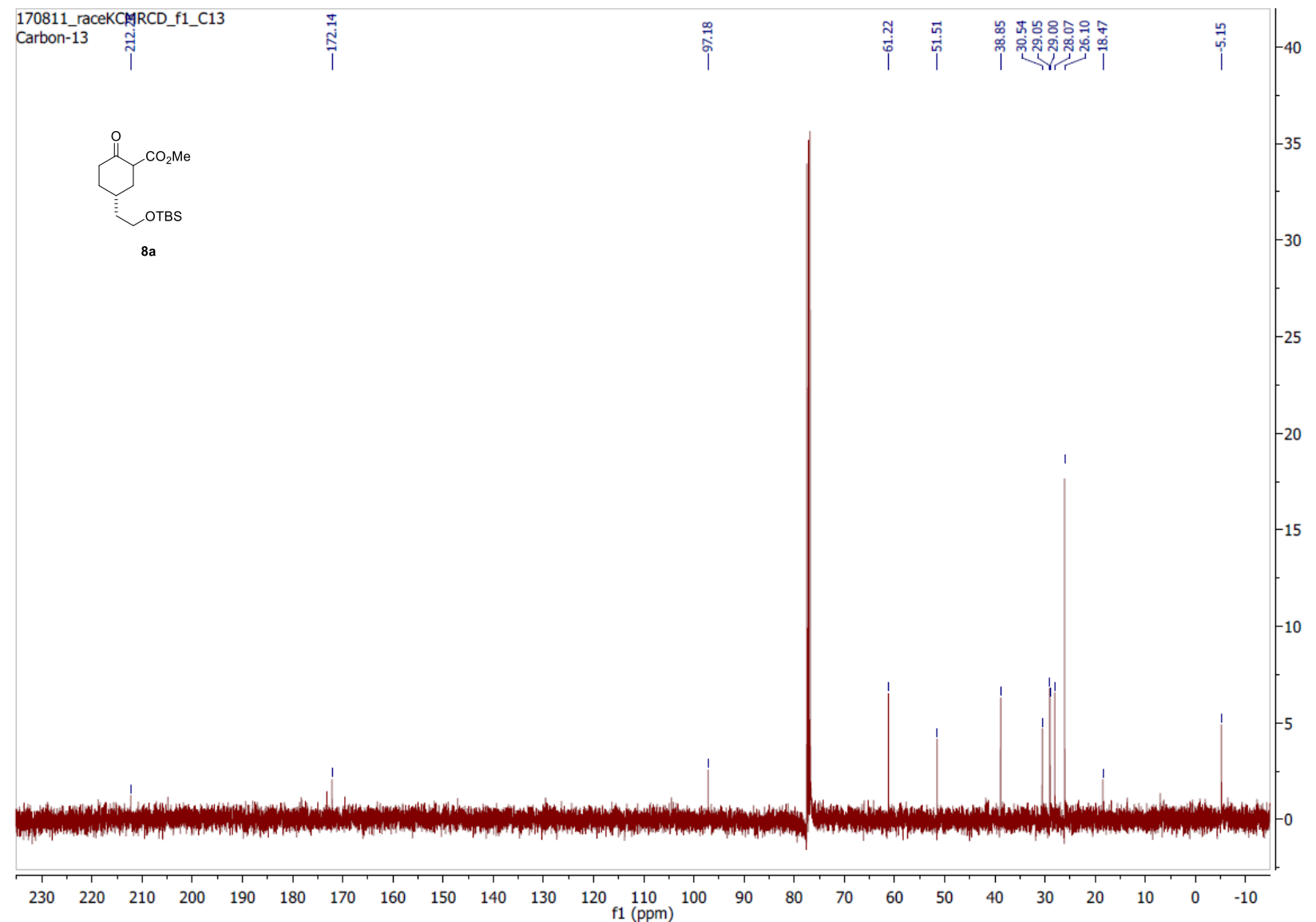


1H NMR (CDCl3, $401 \mathrm{MHz})$ :

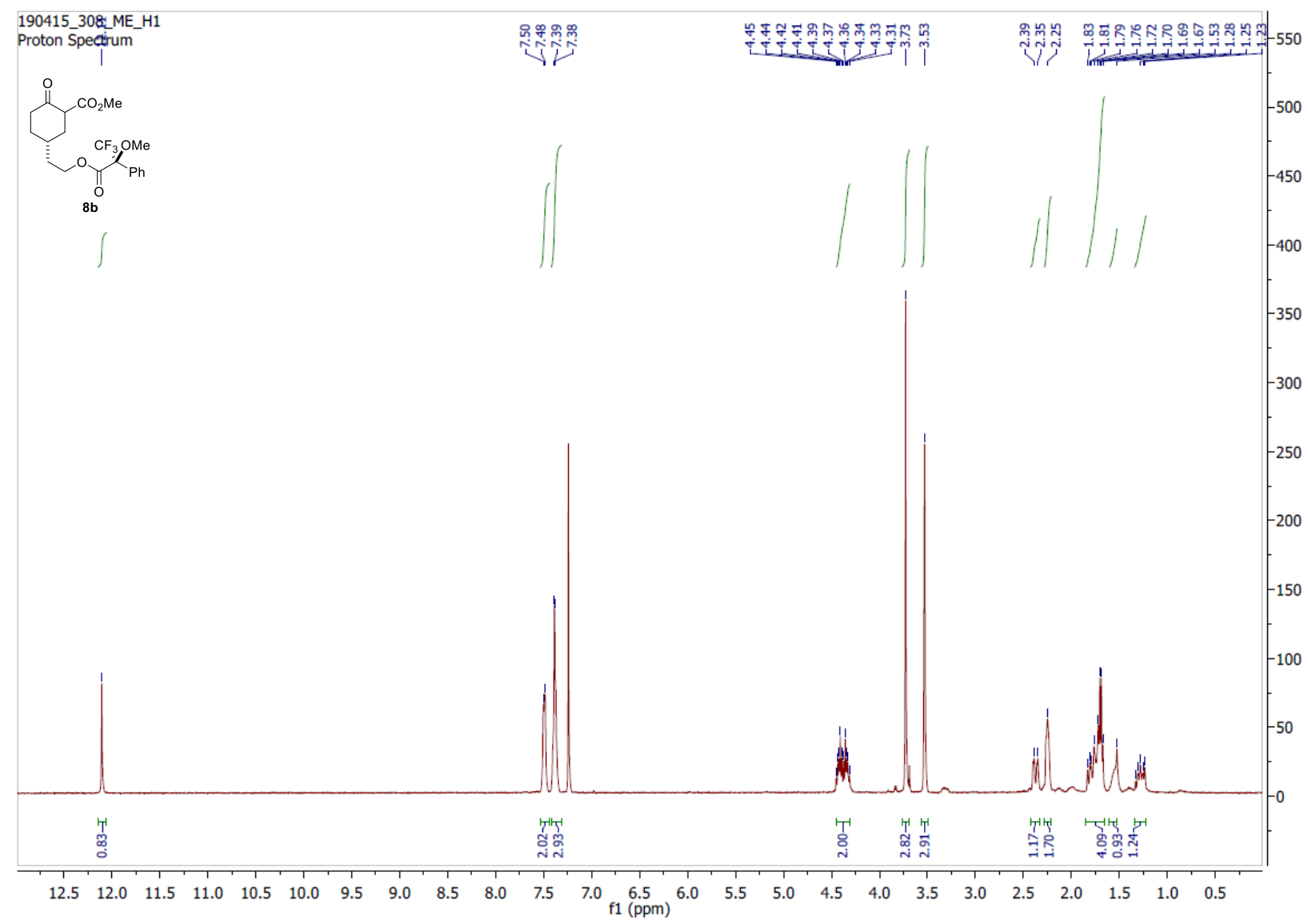


13C $\{1 \mathbf{H}\}$ NMR $(\mathrm{CDCl} 3,101 \mathrm{MHz}):$

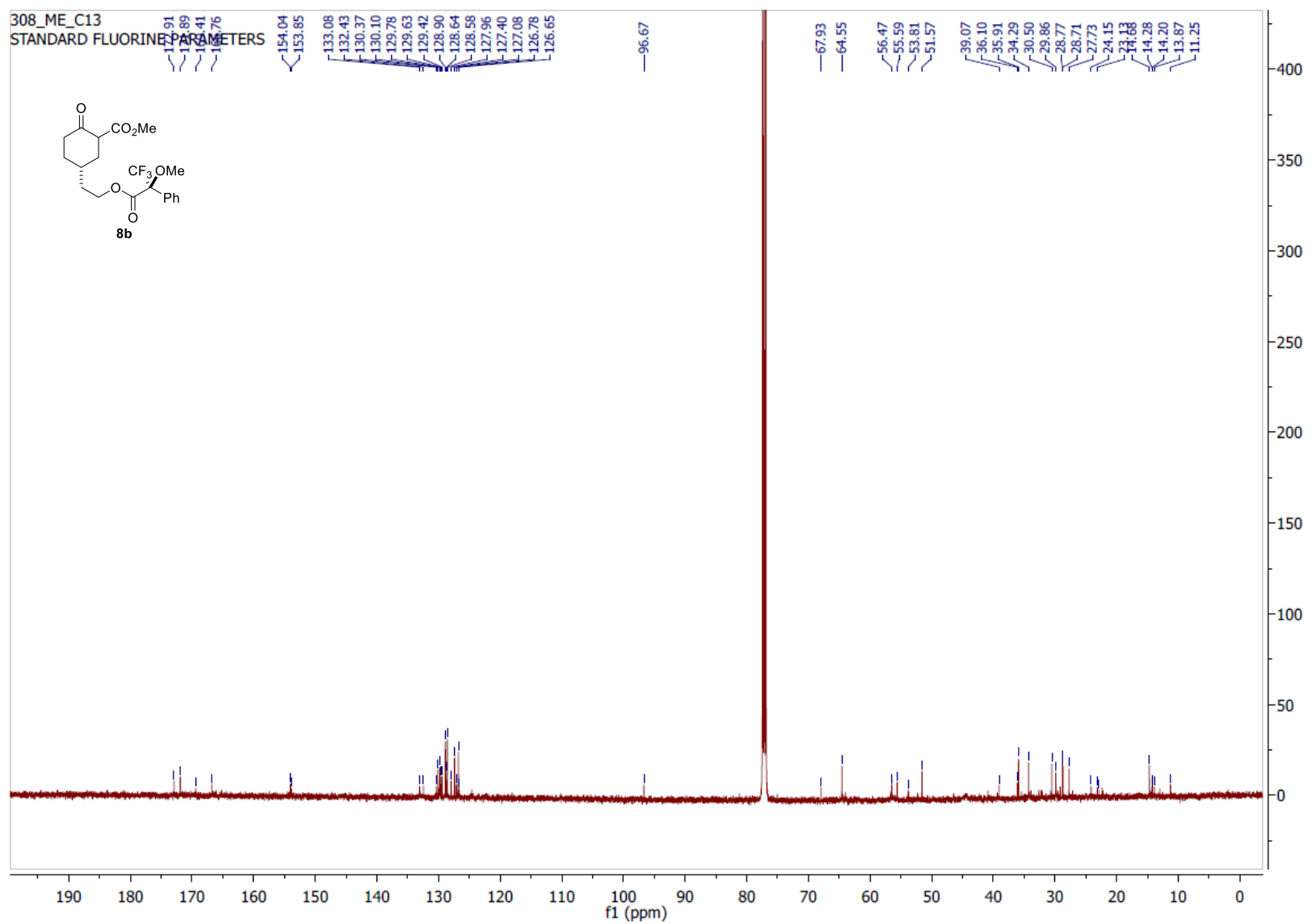


19F NMR (CDCl3, $377 \mathrm{MHz})$ :
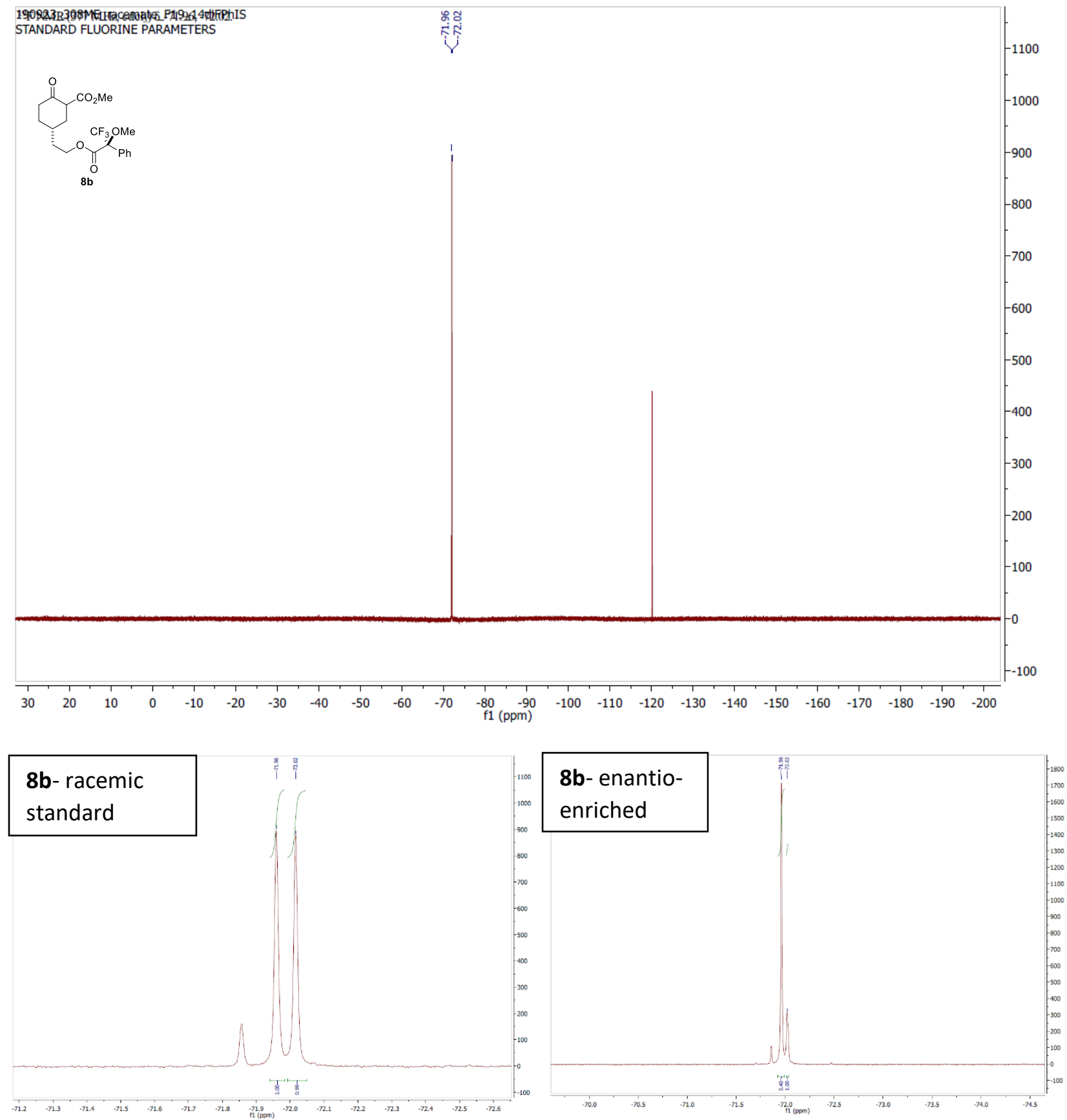
1H NMR (CDCl3, $700 \mathrm{MHz})$ :

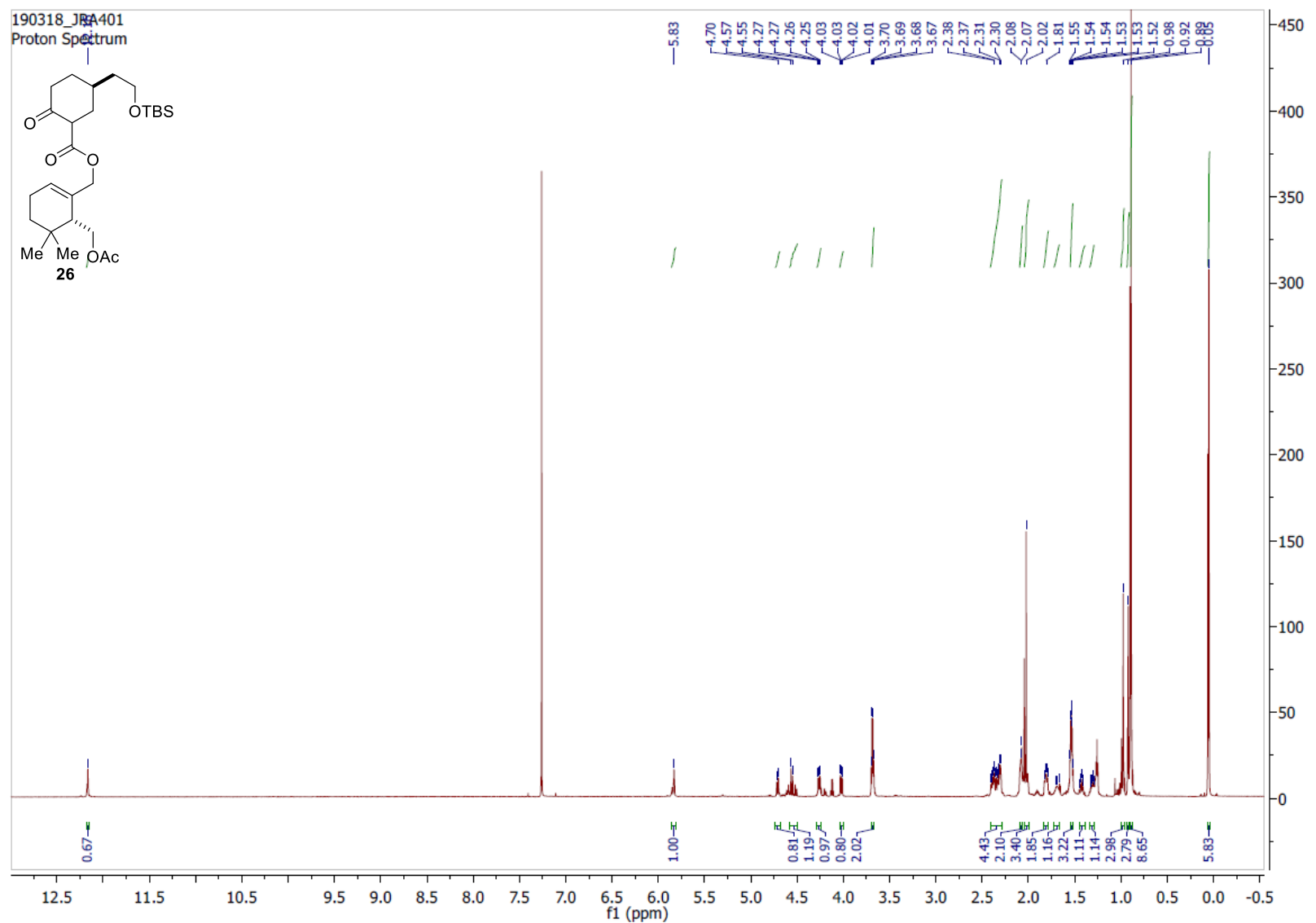


13C $\{1$ H $\}$ NMR (CDCl3, $126 \mathrm{MHz})$ :

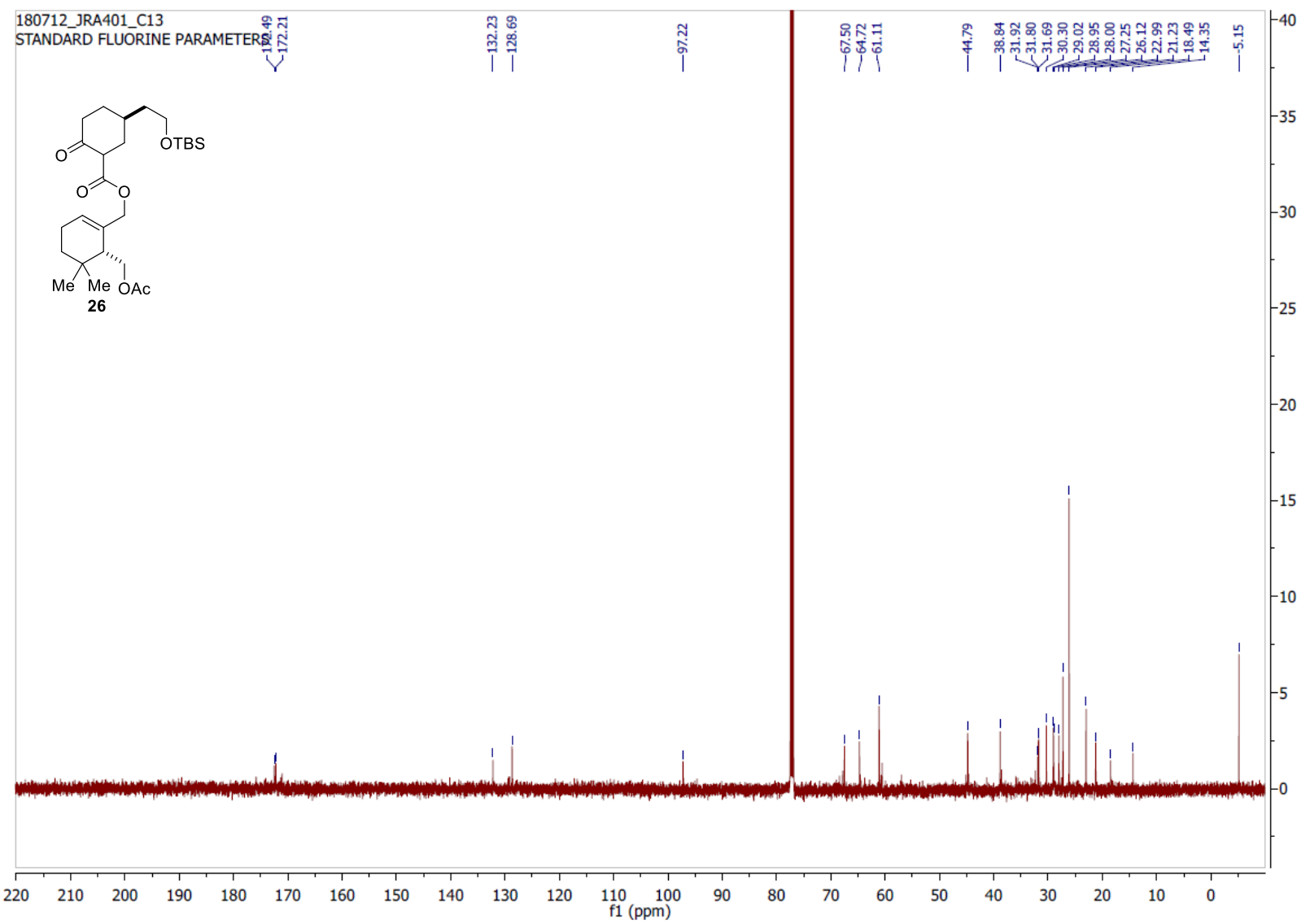


1H NMR (CDCl3, $401 \mathrm{MHz})$ :

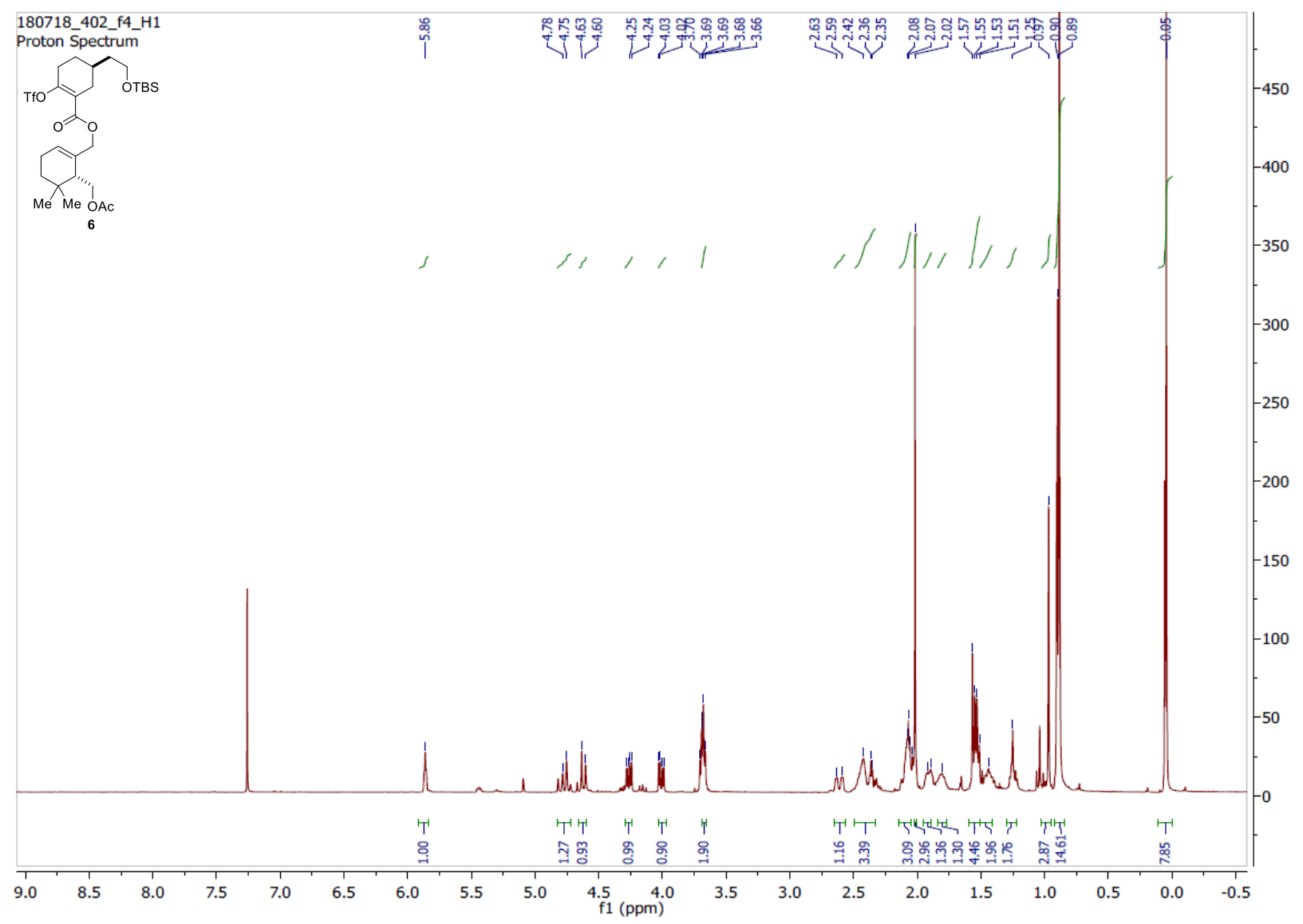


13C $\{1$ H $\}$ NMR (CDCl3, $126 \mathrm{MHz}):$

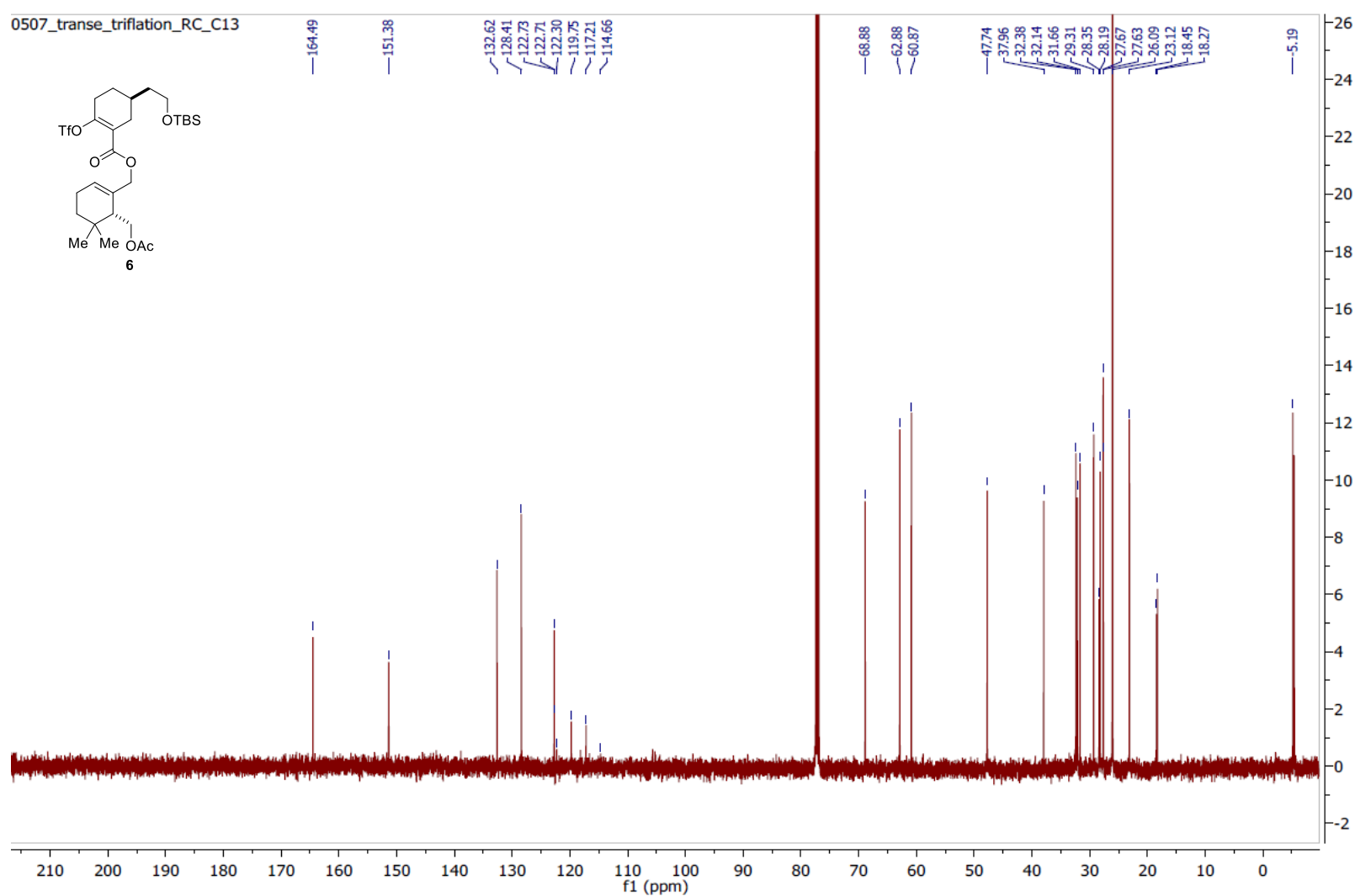


1H NMR (CDCl3, $400 \mathrm{MHz})$ :

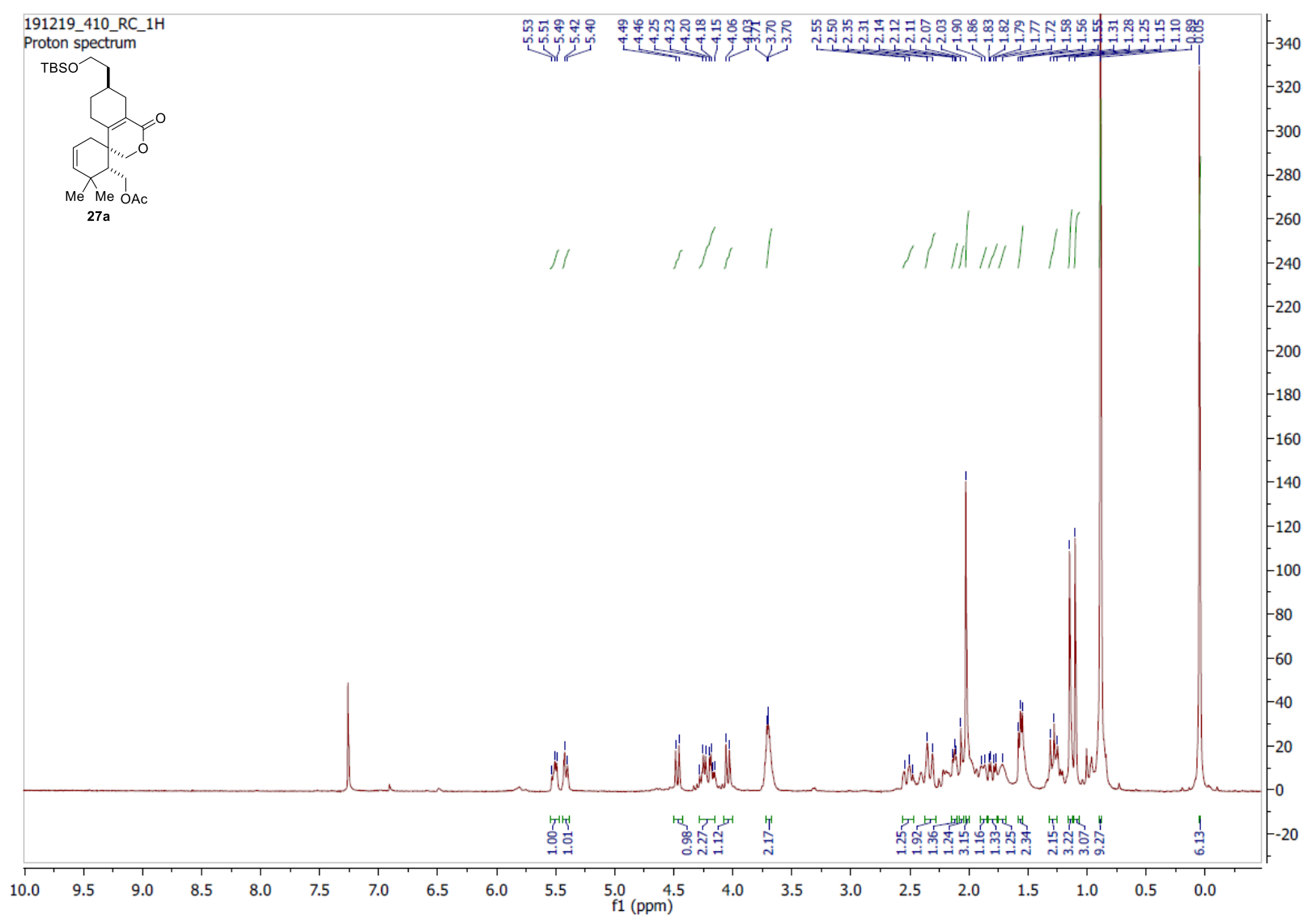


13C $\{1$ H $\}$ NMR (CDCl3, $126 \mathrm{MHz})$ :

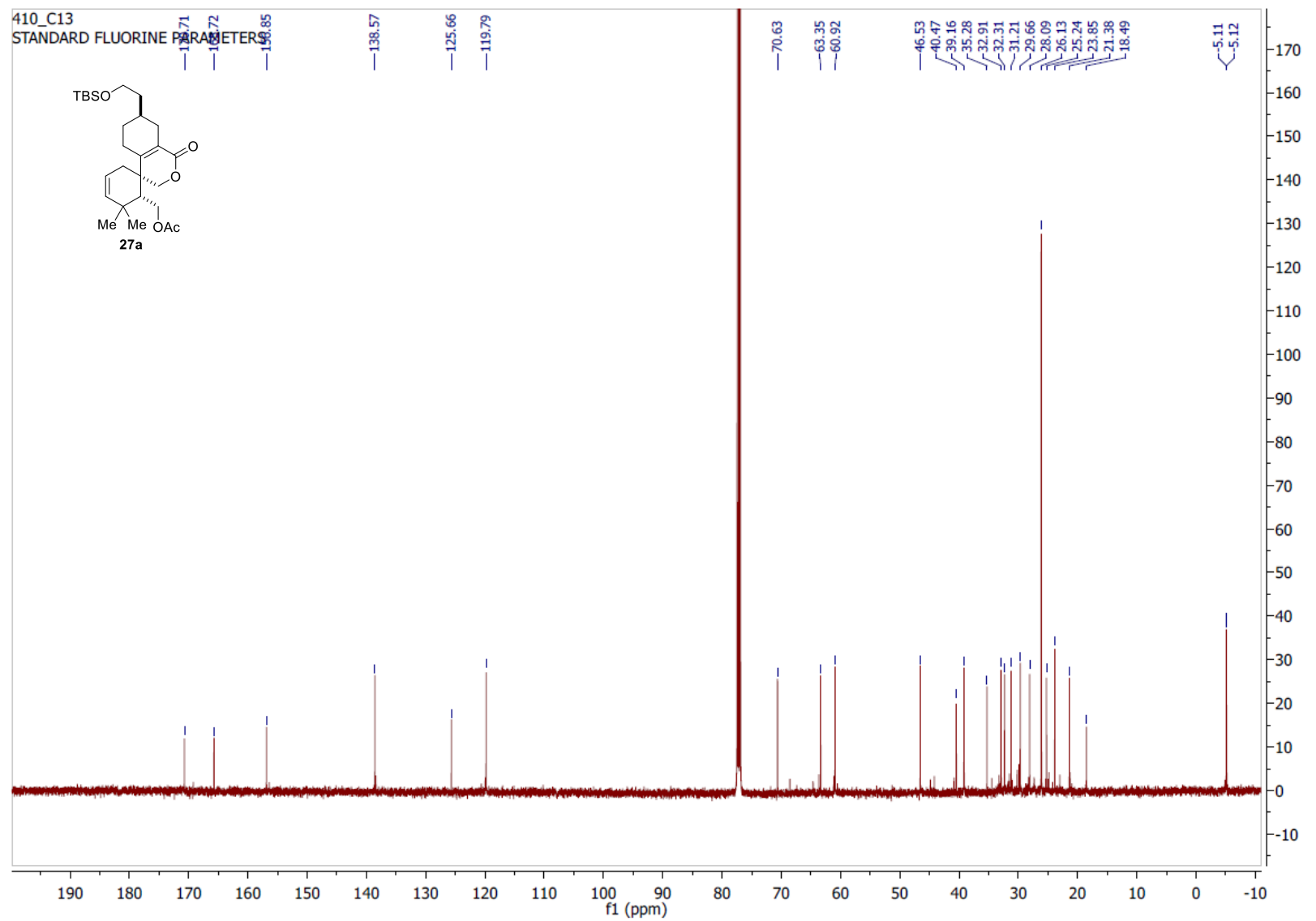


1H NMR (CDCl3, $700 \mathrm{MHz}$ ):

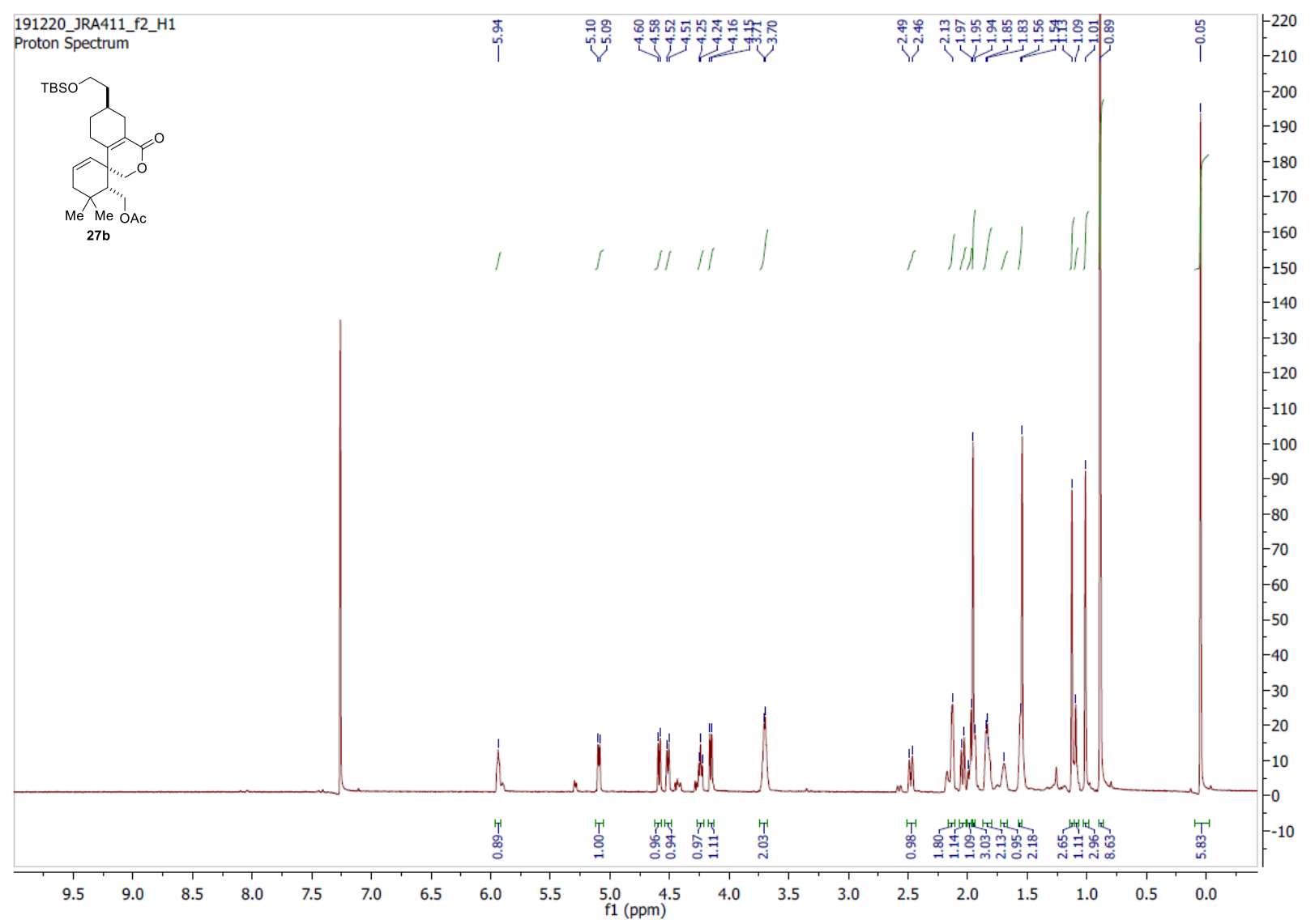


13C $\{1$ H $\}$ NMR (CDCl3, $176 \mathrm{MHz})$ :

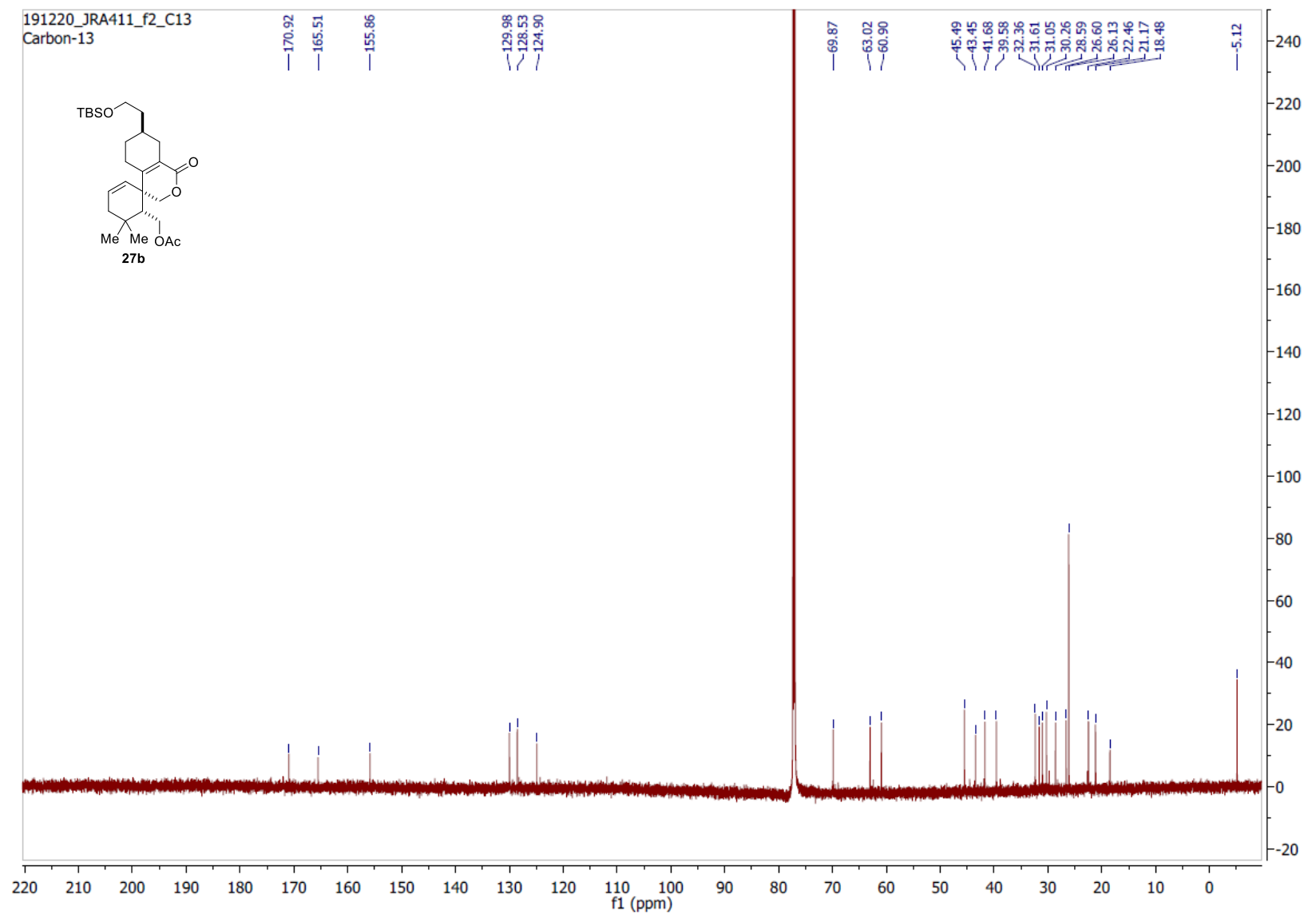


1H NMR (CDCl3, $700 \mathrm{MHz}$ ):

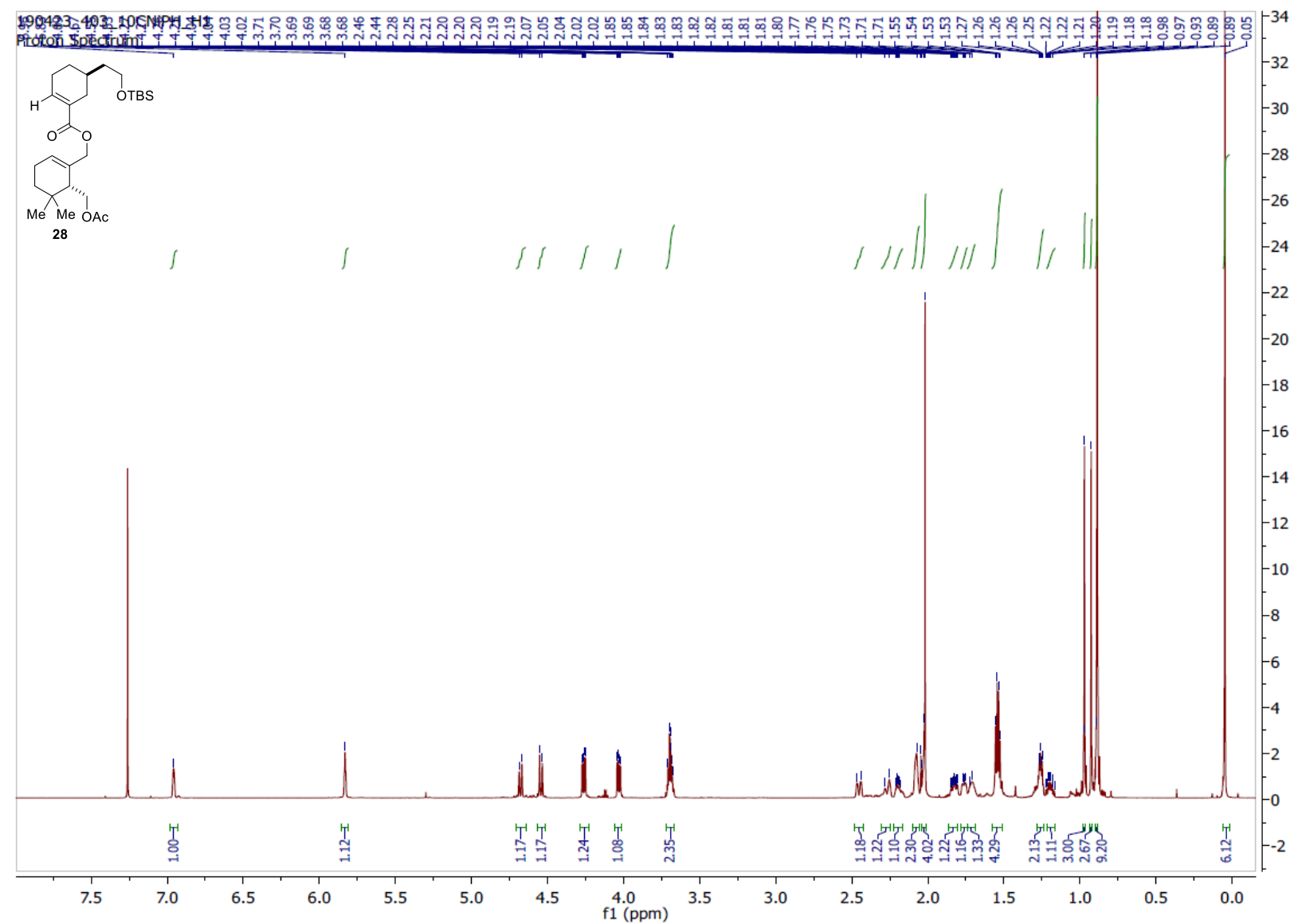


13C $\{1$ H $\}$ NMR (CDCl3, $176 \mathrm{MHz})$ :

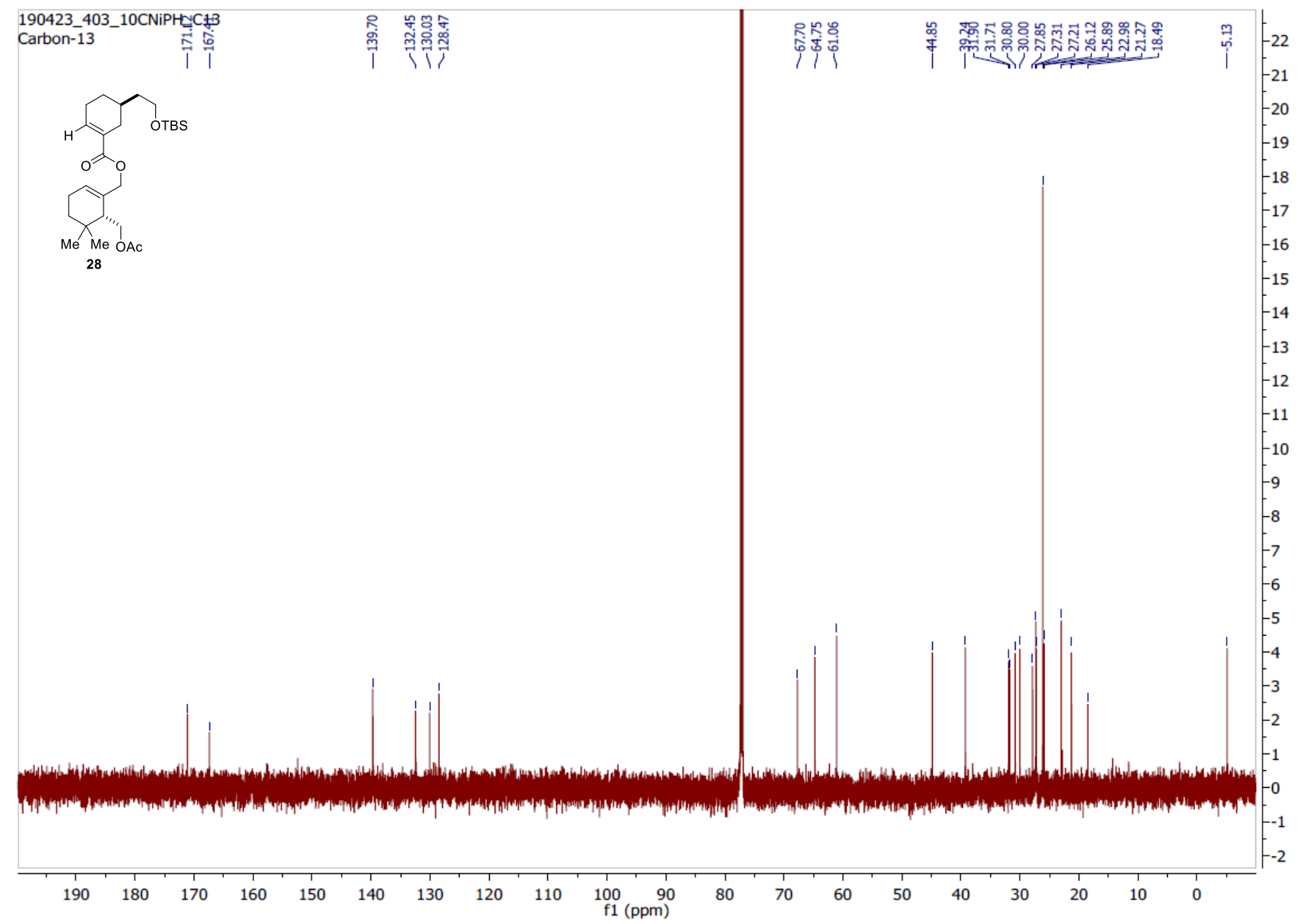


1H NMR (CDCl3, $400 \mathrm{MHz})$ :

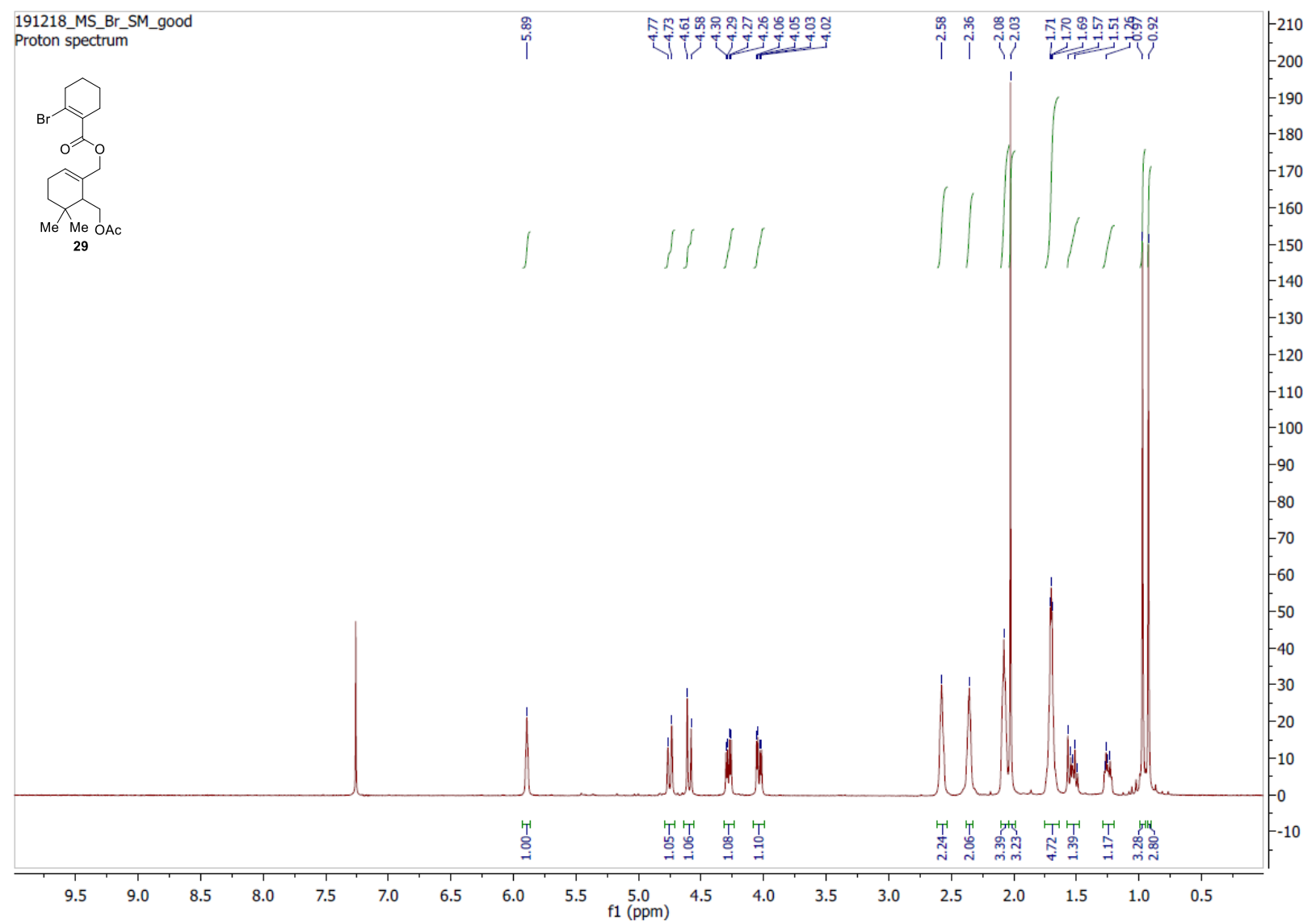


13C $\{1$ H $\}$ NMR (CDCl3, $176 \mathrm{MHz})$ :

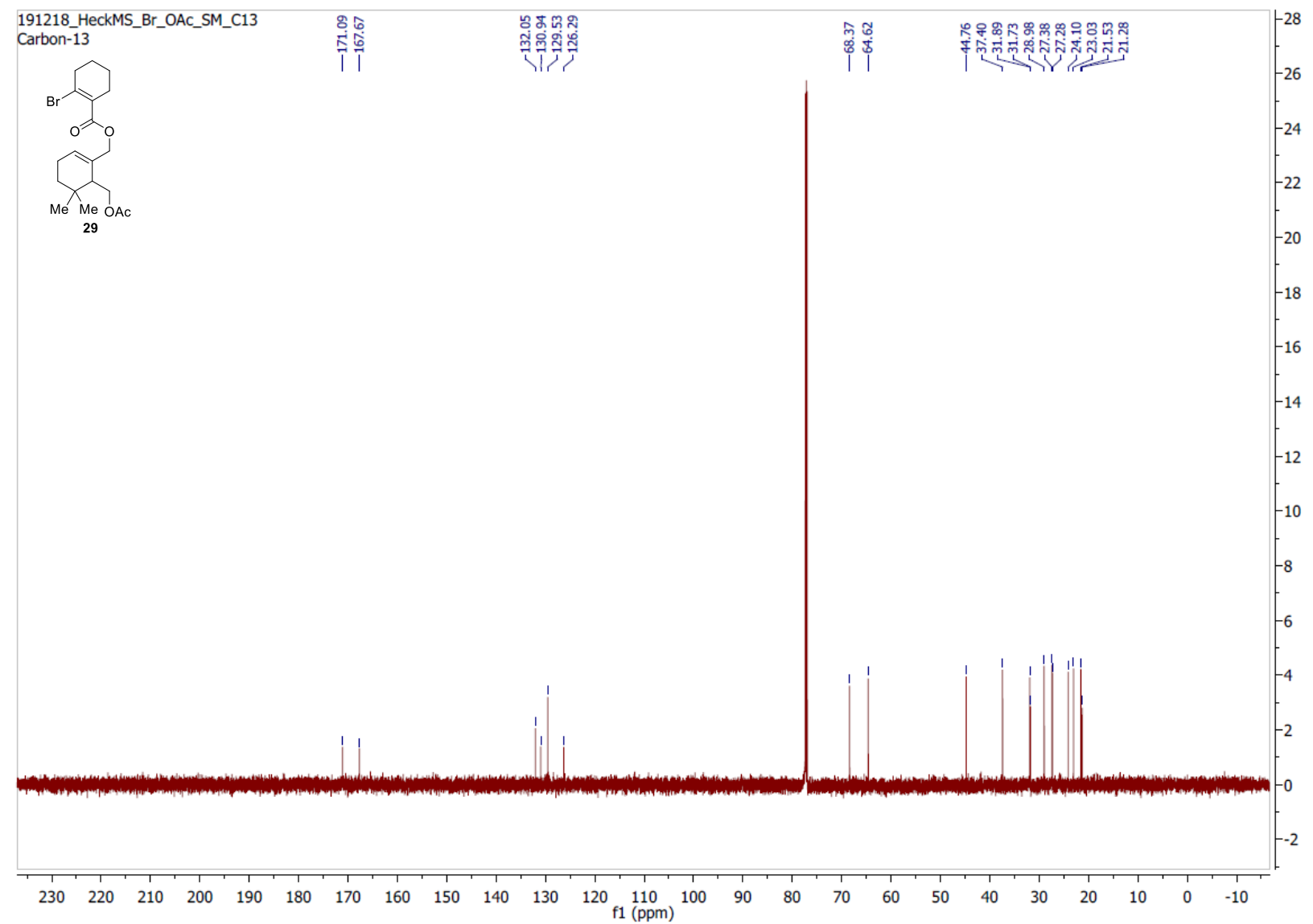


1H NMR (CDCl3, $700 \mathrm{MHz}$ ):

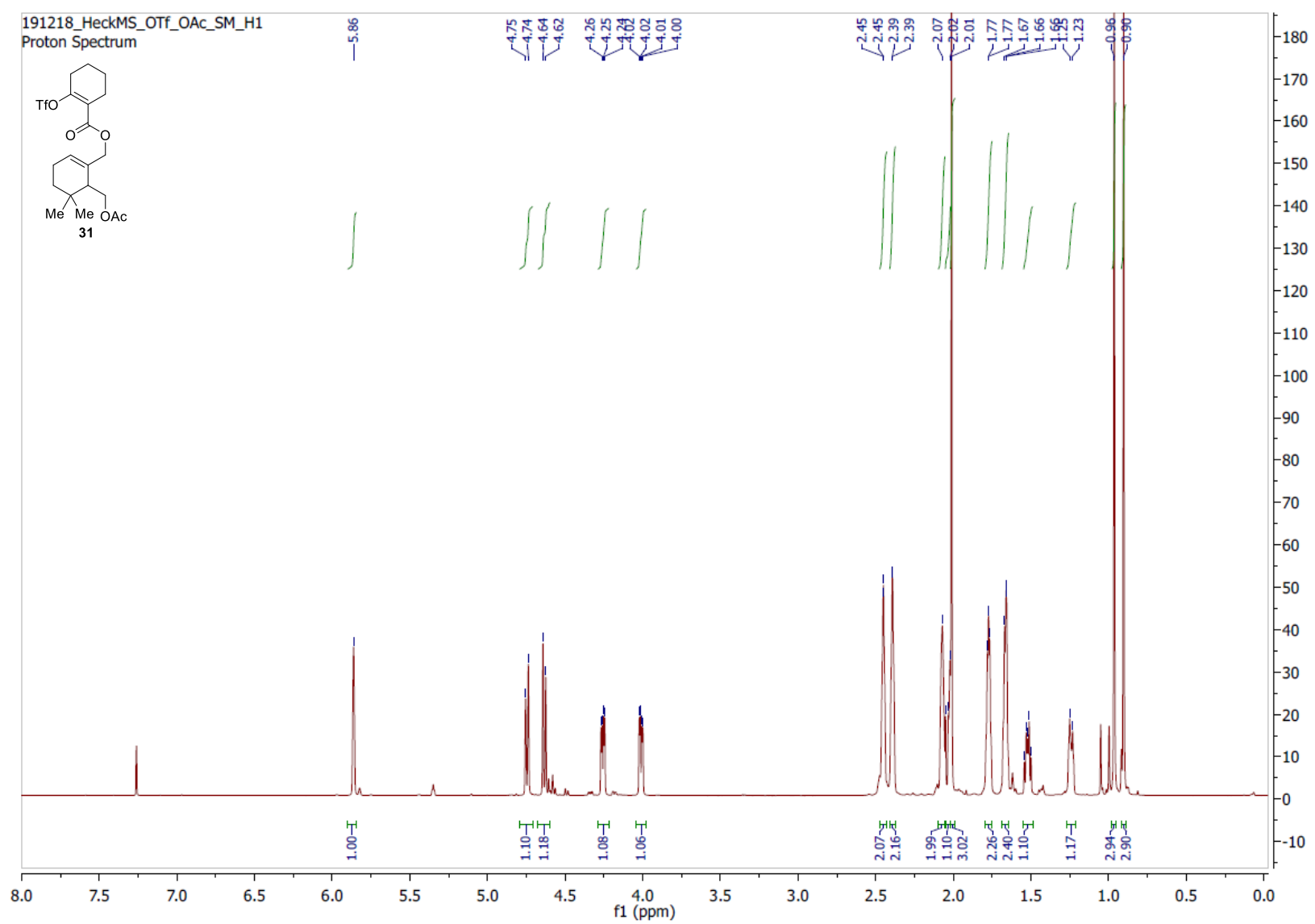


13C $\{1$ H $\}$ NMR (CDCl3, $176 \mathrm{MHz})$ :

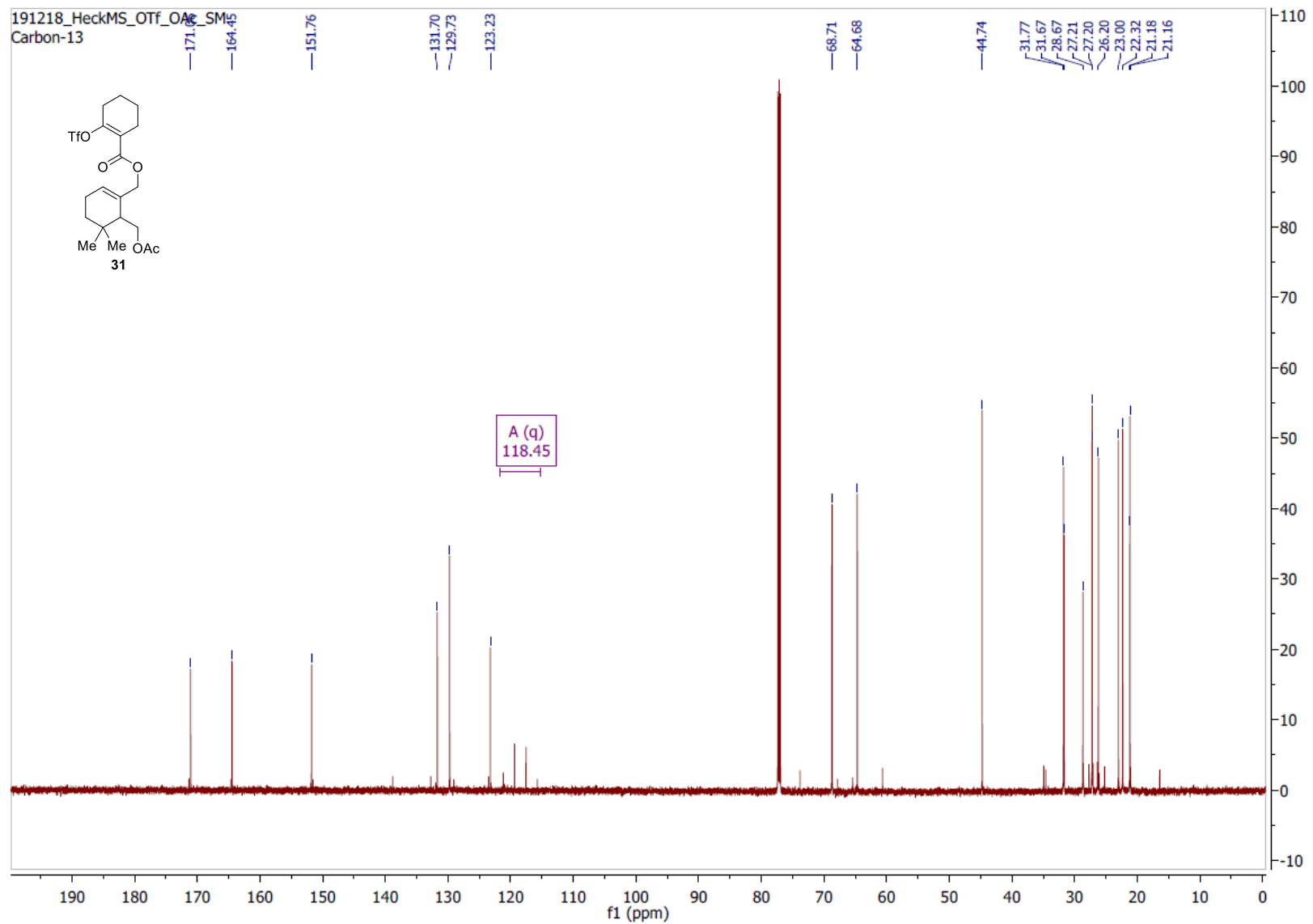


1H NMR (CDCl3, $700 \mathrm{MHz})$ :

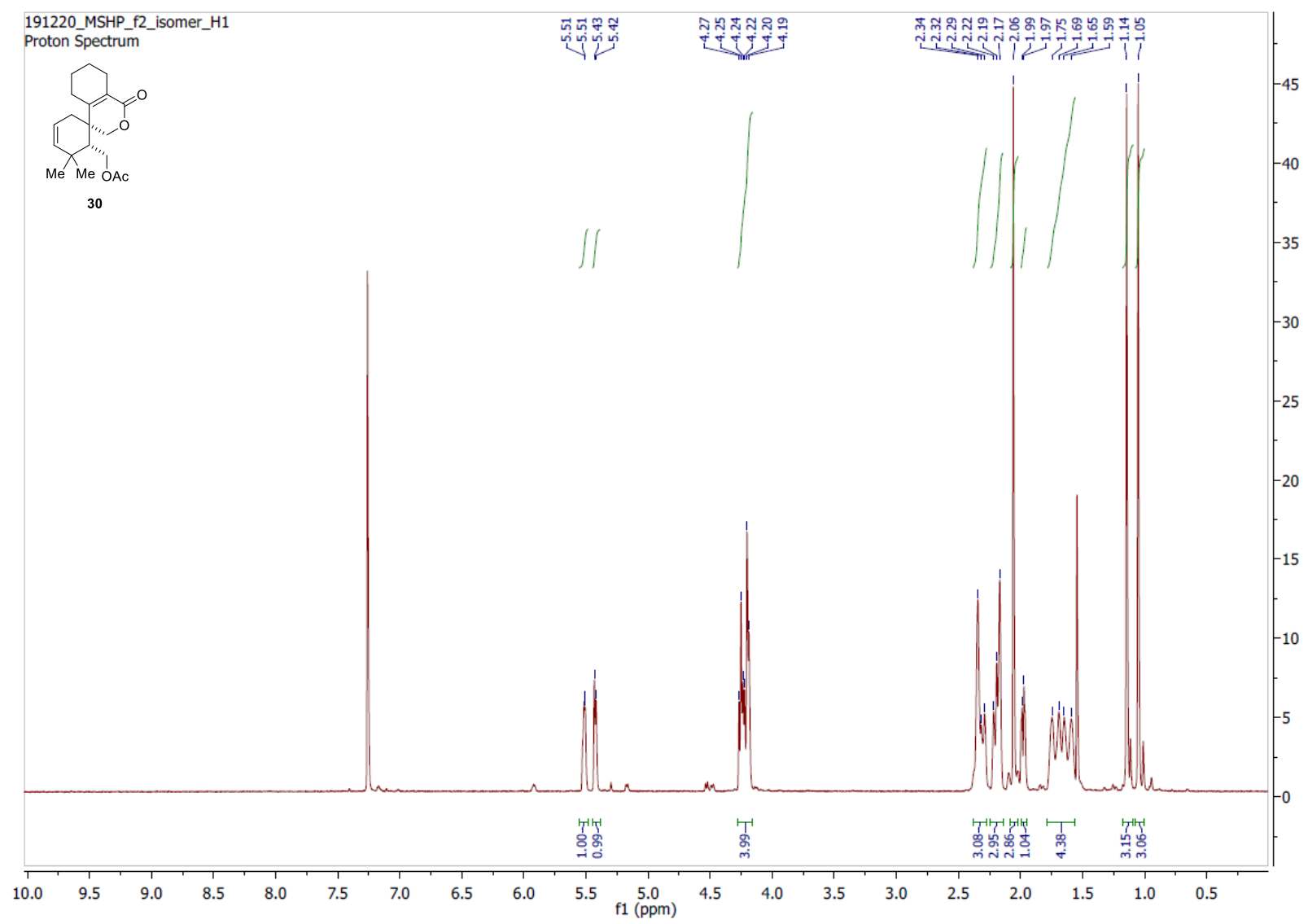


13C $\{1$ H $\}$ NMR (CDCl3, $176 \mathrm{MHz})$ :

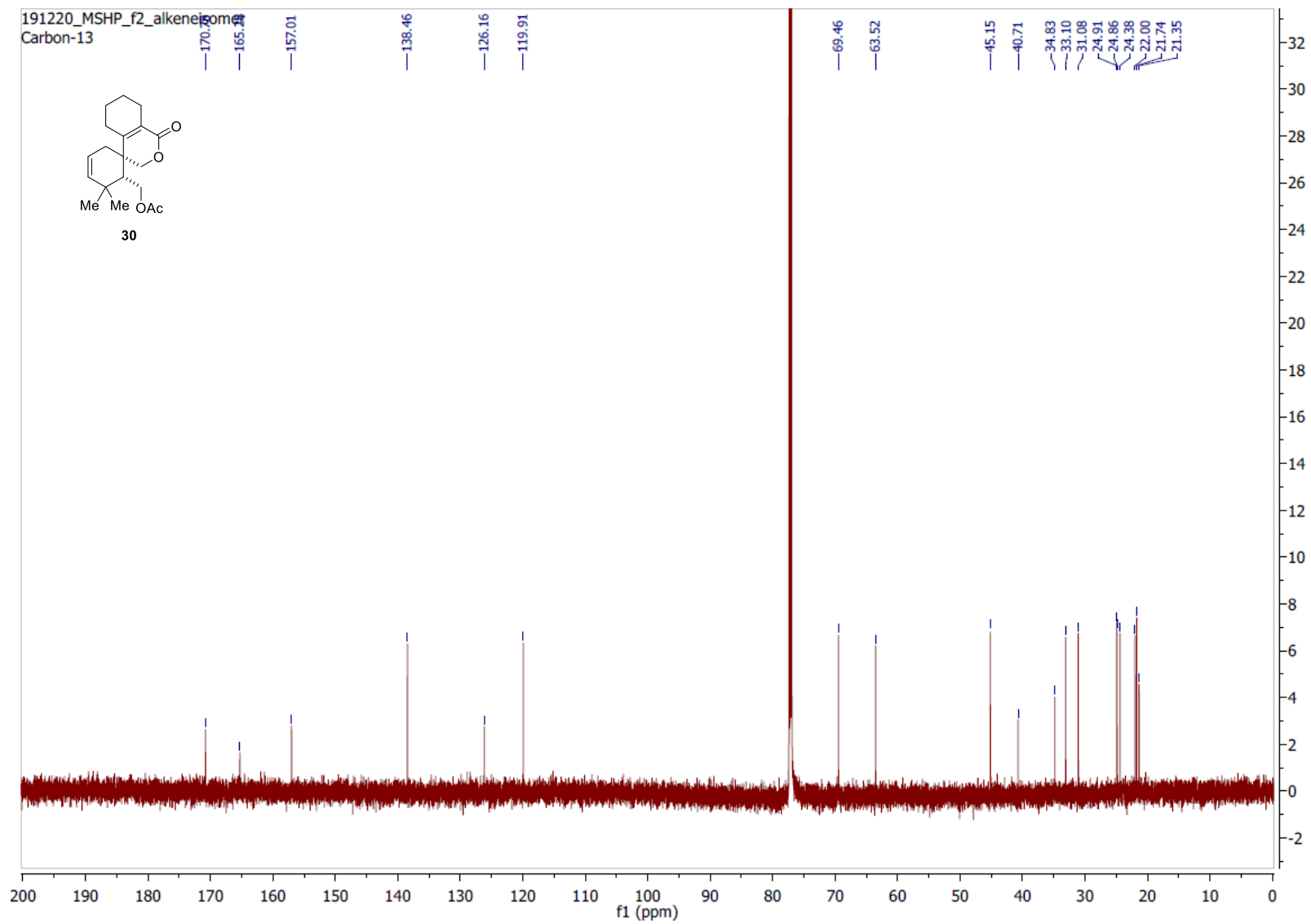




\section{Chiral HPLC Trace}<smiles>CC1=CCCC(C)(C)[C@H]1COC(C)(C)C</smiles>

(R)-(2,6,6-trimethylcyclohex-2-en-1-yl)methyl benzoate which was prepared by dissolving a small aliquot of crude cyclogeraniol (9) $(50 \mathrm{mg}, 0.19 \mathrm{mmol})$, triethyl amine (21 mg, $29 \mu \mathrm{L}, 0.21 \mathrm{mmol}, 1.1 \mathrm{equiv}$.), and benzoyl chloride ( $24 \mathrm{mg}, 20 \mu \mathrm{L}, 0.17 \mathrm{mmol}, 0.9$ equiv.) in DCM $(0.5 \mathrm{~mL})$ and the reaction was stirred at room temperature for $4 \mathrm{~h}$. The reaction mixture was quenched with saturated $\mathrm{NaHCO}_{3}(5 \mathrm{~mL})$, extracted with DCM (5 mL x 2), dried over anhydrous $\mathrm{Na}_{2} \mathrm{SO}_{4}$, concentrated, and purified by flash column chromatography on silica gel (10\% ethyl acetate/hexanes) to yield benzolated analog as a colorless oil (44 mg, 90\% yield). 1H NMR (CDCl3, $400 \mathrm{MHz}): \delta 8.03$ (d, $J=8.0 \mathrm{~Hz}, 2 \mathrm{H}), 7.55(\mathrm{t}, J=7.4 \mathrm{~Hz}, 1 \mathrm{H}), 7.44$ $(\mathrm{t}, J=7.7 \mathrm{~Hz}, 2 \mathrm{H}), 5.50(\mathrm{~s}, 1 \mathrm{H}), 4.38(\mathrm{~d}, J=4.6 \mathrm{~Hz}, 2 \mathrm{H}), 2.03(\mathrm{~s}, 2 \mathrm{H}), 1.95(\mathrm{~s}, 1 \mathrm{H}), 1.80(\mathrm{~s}, 3 \mathrm{H}), 1.57(\mathrm{dt}$, $J=13.3,8.7 \mathrm{~Hz}, 1 \mathrm{H}), 1.29-1.22(\mathrm{~m}, 1 \mathrm{H}), 1.03(\mathrm{~s}, 3 \mathrm{H}), 0.97(\mathrm{~s}, 3 \mathrm{H})$.

Racemic HPLC Trace (ChiralPak IA column, 4.6x250mm; gradient $0.1 \%-0.2 \%$ isopropanol in hexanes over 10 minutes):

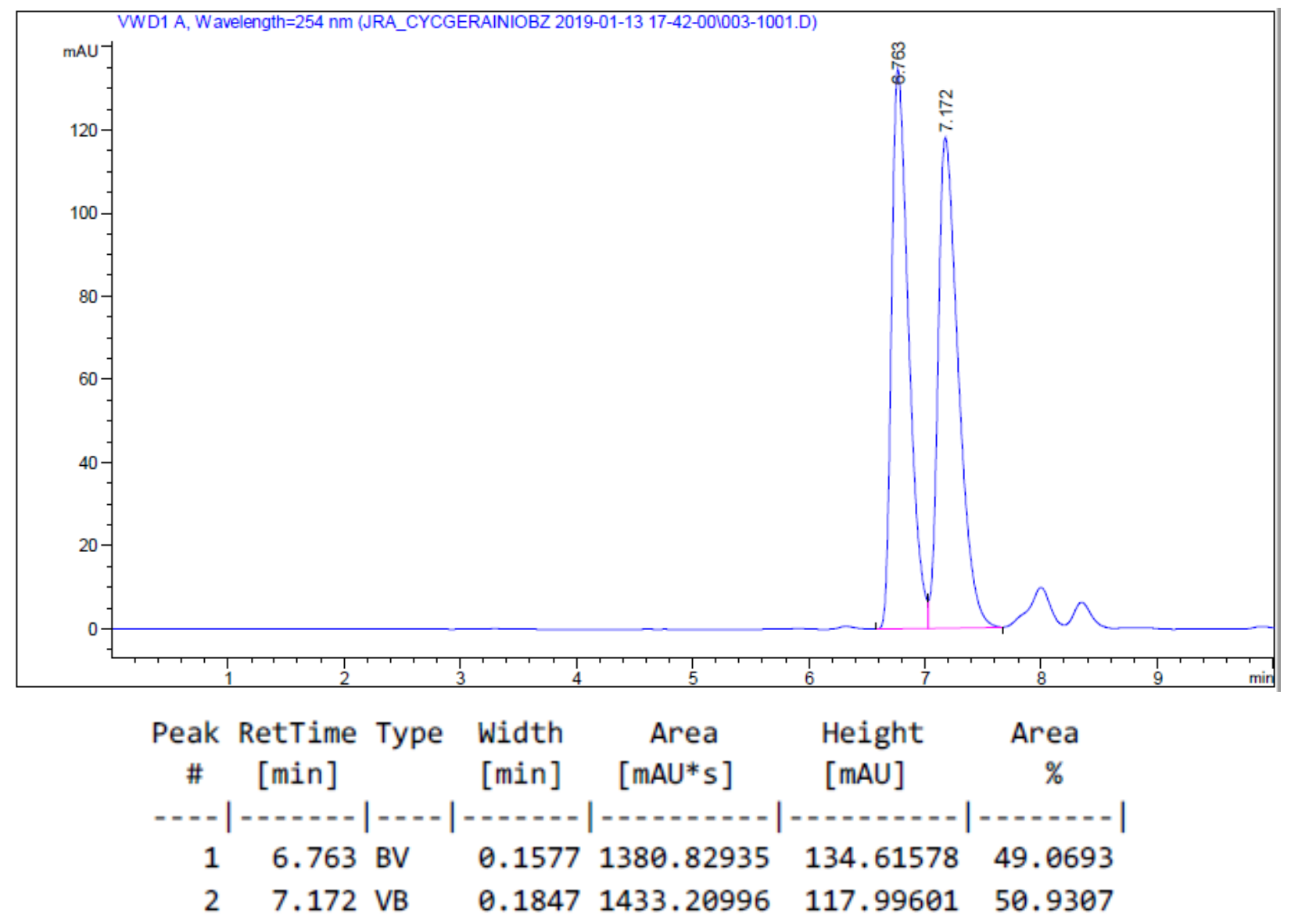


Enantioenriched HPLC Trace:

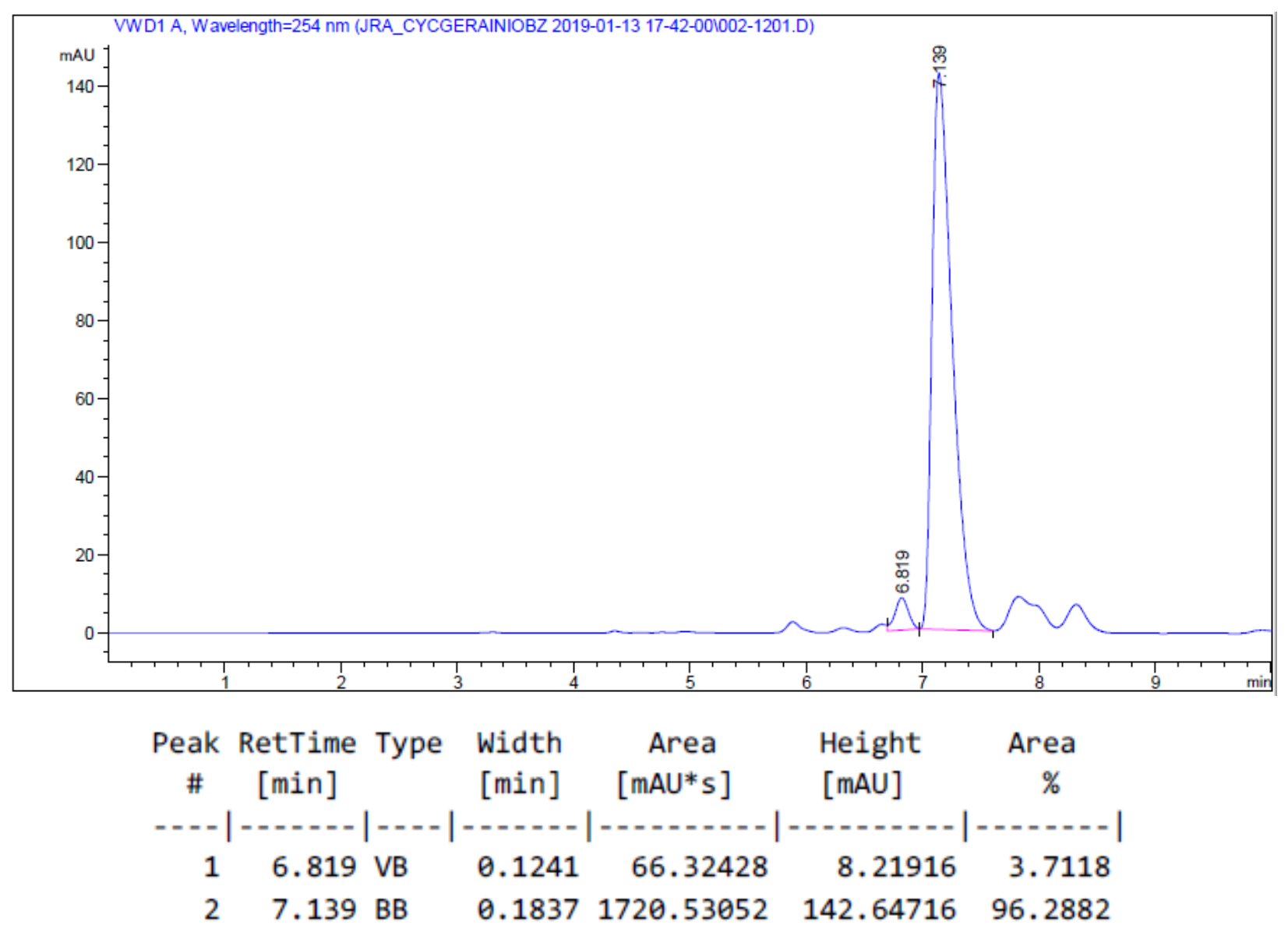

